UCI-TR-02-37

YITP-02-64

hep-ph/0210390

\title{
Supersymmetry and the Linear Collider
}

\author{
Jonathan L. Feng ${ }^{a}$ and Mihoko M. Nojiri ${ }^{b}$ \\ ${ }^{a}$ Department of Physics and Astronomy \\ University of California, Irvine, CA 92697, USA \\ ${ }^{b}$ Yukawa Institute of Theoretical Physics \\ Kyoto University, Kyoto 606-8502, Japan
}

\begin{abstract}
We present a pedagogical introduction to supersymmetry and supersymmetric models and give an overview of the potential of the linear collider for studying them. If supersymmetry is found, its discovery will bring with it many more questions than answers. These include:

Are the newly discovered particles really superpartners?

If not all superpartners are discovered, where are the rest of them?

Do the electromagnetic, weak, and strong forces unify?

Is a supersymmetric particle the dark matter?

How are the supersymmetric flavor and CP problems solved?

What is the scale of supersymmetry breaking?

What are the fundamental interactions at the Planck scale? We review how the linear collider will provide definitive answers to some of these and may shed light on the rest, even if only one or a few superpartners are kinematically accessible.
\end{abstract}

To appear as a chapter in

Linear Collider Physics in the New Millennium

published by World Scientific

editors David Miller, Keisuke Fujii and Amarjit Soni 


\section{Contents}

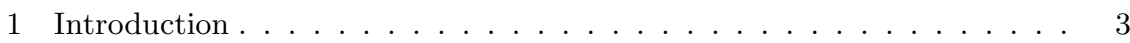

2 The Minimal Supersymmetric Standard Model . . . . . . . . . . . . . . 4

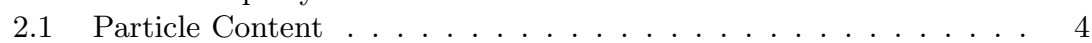

2.2 Supersymmetric Matter Interactions . . . . . . . . . . . . . . . . . 7

2.3 Supersymmetric Gauge Interactions . . . . . . . . . . . . . . . . . . . . . . . 9

2.4 Supersymmetry-breaking Terms . . . . . . . . . . . . . . . . . . . 10

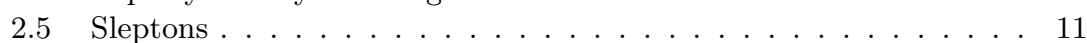

2.6 Charginos and Neutralinos . . . . . . . . . . . . . . . . . . 13

3 Successes and Puzzles $\ldots \ldots \ldots \ldots \ldots \ldots$

3.1 Unification . . . . . . . . . . . . . . . . . . 15

3.2 Dark Matter . . . . . . . . . . . . . . . . . . . . . . . . . . . . . . . . . 17

$3.3 \quad$ Flavor Violation . . . . . . . . . . . . . . . . . . . . . . . . . . . . 17

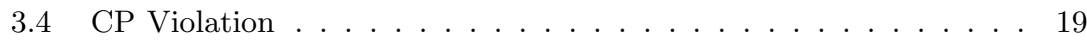

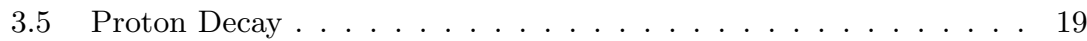

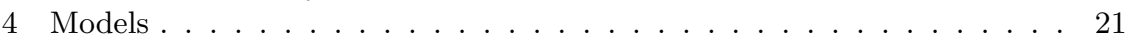

4.1 Minimal Supergravity . . . . . . . . . . . . . . . . . . . . . 23

4.2 Focus Point Supersymmetry . . . . . . . . . . . . . . . . . . . . . 25

4.3 Superheavy Supersymmetry . . . . . . . . . . . . . . . . . . . . . 28

4.4 Gauge Mediation . . . . . . . . . . . . . . . . . . . . 28

4.5 Anomaly Mediation ... . . . . . . . . . . . . . . . . . 30

4.6 GUT and Planck Scale Frameworks . . . . . . . . . . . . . . . . 32

5 Slepton Studies . . . . . . . . . . . . . . . . . . . . 33

5.1 Signal and Background . . . . . . . . . . . . . . . . . . . 34

5.2 Slepton Masses . . . . . . . . . . . . . . . . . . . . . . 36

5.3 Polarized Cross Sections . . . . . . . . . . . . . . . . . . . . . 41

5.4 Lepton Flavor Violation . . . . . . . . . . . . . . . . . . . . . . 45

5.5 Tau Polarization from Stau Decay . . . . . . . . . . . . . . . . . . . . 47

6 Chargino and Neutralino Studies . . . . . . . . . . . . . . . . . . 49

6.1 Signal and Background . . . . . . . . . . . . . . . . . . . . 49

6.2 Masses and Polarized Cross Sections . . . . . . . . . . . . . . . . 51

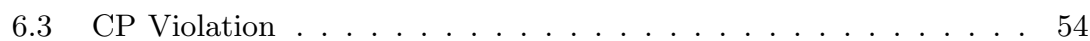

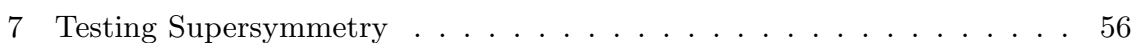

7.1 Verifying Supersymmetry . . . . . . . . . . . . . . . . . . 57

7.2 Super-oblique Parameters . . . . . . . . . . . . . . . . . 59

8 Determining the Scale of Supersymmetry Breaking . . . . . . . . . . . . 61

9 Extrapolation to the Planck Scall $\ldots \ldots \ldots$. . . . . . . . . . 63

10 Connections to Cosmology . . . . . . . . . . . . . . . . . . 65

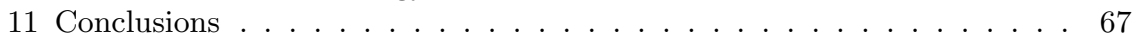

Acknowledgements . . . . . . . . . . . . . . . . . . . . . . 68

References. . . . . . . . . . . . . . . . . . . . . . . . 69

Index . . . . . . . . . . . . . . . . . . . . . . . . . . . . . . 74 


\section{Introduction}

Supersymmetry relates fermions to bosons and postulates the existence of a partner particle for every known particle. Its motivations range from the gauge hierarchy problem and the central role of supersymmetry in quantum theories of gravity to the unification of gauge couplings and the existence of dark matter. The search for supersymmetry in particle physics has been carried out on numerous fronts, and its discovery would mark the culmination of an intense research effort spanning several decades.

At the same time, the discovery of supersymmetry will bring with it many more questions than answers. These include:

- Are the newly discovered particles really superpartners?

- If not all superpartners are discovered, where are the rest of them?

- Do the electromagnetic, weak, and strong forces unify?

- Is a supersymmetric particle the dark matter?

- How are the supersymmetric flavor and CP problems solved?

- What is the scale of supersymmetry breaking?

- What are the fundamental interactions at the Planck scale?

Insights into these and other fundamental questions will have far-reaching implications, transforming not only particle physics, but also astrophysics and cosmology.

Here we present a pedagogical overview of what the linear collider may contribute to answering these questions. The large and growing world-wide interest in linear colliders has motivated hundreds of detailed studies of the potential of linear colliders for measuring supersymmetry parameters. Typically, however, there is little opportunity to review in broad terms the underlying physics questions and overall goals of this program. In this overview, we therefore begin with a brief introduction to supersymmetry in Sec. (2), focusing on the most salient points for collider physics. We then describe several outstanding successes and puzzles driving work in supersymmetry in Sec. (3) and some of the resulting models in Sec. (4). Against this backdrop, we then review what linear colliders may tell us in the remaining sections. An index of key terms is provided at the end. Throughout this overview, we consider an $e^{+} e^{-}$linear collider with centerof-mass energy between $300-1000 \mathrm{GeV}$, a luminosity of $\mathcal{O}(100) \mathrm{fb}^{-1} / \mathrm{yr}$, highly polarizable electron (and possibly also positron) beams, and the capability of running in alternative modes, such as $e^{-} e^{-}, e^{-} \gamma$.

This overview is neither a thorough introduction to supersymmetry nor 
a comprehensive review of the linear collider literature. Supersymmetry and its phenomenological implications have been systematically presented in a number of beautiful introductions. (See, for example, Refs. 11, 2, 3, , 4, 5, 6. 7, 8.) Detailed supersymmetry studies at linear colliders and exhaustive bibliographies may be found in recent reports of the large linear collider collaborations [9, 10, 11].

\section{The Minimal Supersymmetric Standard Model}

The minimal supersymmetric standard model (MSSM) is the supersymmetric extension of the standard model with minimal field content, conserved $R$-parity, and no additional theoretical assumptions. It is the underlying framework for most "model-independent" collider studies, and we therefore begin by describing its particle content and interactions.

\subsection{Particle Content}

Supersymmetry is, under general assumptions, the unique extension of the Poincare algebra, the algebra of spacetime translations $P$, rotations $J$, and boosts $K$. In its simplest form, supersymmetry extends the Poincare algebra by introducing two additional operators $Q$ and $\bar{Q}$, which satisfy

$$
\left\{Q_{\alpha}, \bar{Q}_{\dot{\beta}}\right\}=2 \sigma_{\alpha \dot{\beta}}^{\mu} P_{\mu}, \quad\left[Q_{\alpha}, P^{\mu}\right]=\left[\bar{Q}_{\dot{\beta}}, P^{\mu}\right]=0,
$$

where the braces denote anti-commutation, $\sigma^{0}$ is the $2 \times 2$ identity matrix, and the $\sigma^{i}$ are the Pauli sigma matrices. $Q$ and $\bar{Q}$ are twocomponent spinors and fermionic, and so supersymmetry transformations relate fermions to bosons.

If supersymmetry is a fundamental symmetry of nature, all particles must lie in representations of the supersymmetry algebra. In conventional quantum field theory, particles are represented by fields, functions of the four spacetime coordinates. The Poincare algebra is represented by differential operators acting on these fields. For example,

$$
P_{x}=i \frac{\partial}{\partial x}, J_{x}=-i\left(y \frac{\partial}{\partial z}-z \frac{\partial}{\partial y}\right), K_{x}=i\left(t \frac{\partial}{\partial x}+x \frac{\partial}{\partial t}\right) .
$$

To formulate supersymmetric field theories, we must extend this formalism to include supersymmetry. Not surprisingly, given the anti-commutator appearing in Eq. (1), this requires an extension of the usual spacetime coordinates to include new anti-commuting, or Grassmanian, coordinates $\theta_{1}, \theta_{2}$, 
$\bar{\theta}_{1}$ and $\bar{\theta}_{2}$. The supersymmetry operators may then be identified with the differential operators

$$
Q_{\alpha}=\frac{\partial}{\partial \theta^{\alpha}}-i \sigma_{\alpha \dot{\beta}}^{\mu} \bar{\theta}^{\dot{\beta}} \partial_{\mu}, \bar{Q}_{\dot{\beta}}=-\frac{\partial}{\partial \bar{\theta}^{\dot{\beta}}}+i \theta^{\alpha} \sigma_{\alpha \dot{\beta}}^{\mu} \partial_{\mu} .
$$

It is not hard to show that the operators of Eqs. (2) and (3) satisfy the relations of Eq. (11). These operators then act on superfields, functions of $x^{\mu}, \theta$, and $\bar{\theta}$, which are the natural representations of the supersymmetry algebra.

To determine the particle content of these theories, we expand superfields in powers of $\theta$ and $\bar{\theta}$. Conventional four-dimensional fields then appear as the coefficients in this expansion. This is completely analogous to the case of a field in higher spacetime dimensions, which may be decomposed into four-dimensional fields through a Fourier expansion in the extra dimensional coordinates. Four-dimensional fields emerge as coefficients in this expansion, producing a zero-mode field and a tower of Kaluza-Klein states. The case of supersymmetry is similar, with one important difference: the Grassmanian properties of the $\theta$ coordinates imply that the expansion terminates, and so superfields contain only a finite set of four-dimensional particles.

The simplest superfields are chiral superfields, independent of $\bar{\theta}$ (and anti-chiral fields, independent of $\theta$ ). Expanding in $\theta$, ones finds

$$
\Psi(x, \theta)=\phi(x)+\sqrt{2} \theta^{\alpha} \psi_{\alpha}(x)+\theta \theta F(x),
$$

where $\theta \theta \equiv \theta^{\alpha} \theta_{\alpha}$. A chiral superfield then contains a chiral fermion $\psi$ and a complex scalar $\phi$. The $\theta$ coordinates have mass dimension $-1 / 2$. The field $F$ therefore has mass dimension 2 and no renormalizable kinetic term. It contains no physical degrees of freedom and is known as an auxiliary field. It may be removed by applying its equation of motion, but it is often convenient to retain it for reasons to be discussed below.

The quarks and leptons of the standard model are contained in chiral superfields, and so supersymmetry requires the existence of scalar superpartners, the squarks and sleptons. Similarly, the Higgs boson of the standard model is in a chiral superfield, and so supersymmetry requires a fermionic superpartner, the Higgsino. Anomaly cancellation requires that this new fermion be accompanied by additional fermions. In the MSSM this is accomplished by introducing an additional Higgs doublet and the accompanying superpartners. The fields of the chiral supermultiplets in the MSSM, along with their quantum numbers, are listed in Table 1. Note that the gauge quantum numbers of particles in the same supermultiplet are 
Table 1. Particles of the Minimal Supersymmetric Standard Model and their $\mathrm{SU}(3), \mathrm{SU}(2)$, and $\mathrm{U}(1)_{Y}$ quantum numbers. All chiral fermions are left-handed, and the superscript $c$ denotes charge conjugation. We will also use the common alternative notations $\tilde{e}_{L} \equiv \tilde{e}_{L}^{-} \equiv \tilde{e}, \tilde{e}_{R}^{*} \equiv \tilde{e}_{R}^{+} \equiv \tilde{e}^{c}$, and so forth.

\begin{tabular}{|c|l|l|}
\hline Chiral supermultiplets & Quarks & Squarks \\
\hline$Q(3,2,1 / 6)$ & $q=(u, d)$ & $\tilde{q}=(\tilde{u}, \tilde{d})$ \\
$U^{c}(3,1,-2 / 3)$ & $u^{c}$ & $\tilde{u}^{c}$ \\
$D^{c}(3,1,1 / 3)$ & $d^{c}$ & $\tilde{d}^{c}$ \\
\hline & Leptons & Sleptons \\
\hline$L(1,2,-1 / 2)$ & $l=(\nu, e)$ & $\tilde{l}=(\tilde{\nu}, \tilde{e})$ \\
$E^{c}(1,1,1)$ & $e^{c}$ & $\tilde{e}^{c}$ \\
\hline$H_{d}(1,2,-1 / 2)$ & Higgs bosons & Higgsinos \\
\hline$H_{u}(1,2,1 / 2)$ & $\left(H_{d}^{0}, H_{d}^{-}\right)$ & $\left(\tilde{H}_{d}^{0}, \tilde{H}_{d}^{-}\right)$ \\
\hline \hline Vector supermultiplets & $\left(H_{u}^{+}, H_{u}^{0}\right)$ & $\left(\tilde{H}_{u}^{+}, \tilde{H}_{u}^{0}\right)$ \\
\hline$(8,1,1)$ & $g a u g e$ bosons & Gauginos \\
$(1,3,1)$ & $W^{ \pm}, Z$ & $\tilde{g}($ gluino $)$ \\
$(1,1,1)$ & $\gamma$ & $\tilde{W}^{ \pm}, \tilde{W}^{0}($ Winos $)$ \\
\hline \hline Gravity supermultiplet & Graviton & Gravitino \\
\hline$(1,1,1)$ & $g_{\mu \nu}$ & $\tilde{G}$ \\
\hline
\end{tabular}

identical, since standard model gauge symmetries are 'internal symmetries,' independent of spacetime symmetries.

Vector supermultiplets are superfields that are functions of both $\theta$ and $\bar{\theta}$. While there are in general nine terms in the expansion, many of them are not physical, as they may be removed by a supersymmetric generalization of gauge transformations. In the end, we are left with only

$$
V(x, \theta, \bar{\theta})=\theta \sigma^{\mu} \bar{\theta} v_{\mu}(x)-i \bar{\theta} \bar{\theta} \theta^{\alpha} \lambda_{\alpha}(x)+i \theta \theta \bar{\theta}_{\dot{\alpha}} \bar{\lambda}^{\dot{\alpha}}(x)+\frac{1}{2} \theta \theta \bar{\theta} \bar{\theta} D(x) .
$$

A vector superfield contains a vector boson $v$ and a Majorana fermion $\lambda$. The field $D$ has mass dimension 2 and so, as with $F$, is an auxiliary field with no physical degrees of freedom. The standard model gauge bosons are contained in vector supermultiplets, and so the MSSM also contains their fermionic partners, the gauginos. These are included in Table 1 .

Finally, although not strictly a particle of the standard model, the graviton $g_{\mu \nu}$ is the gauge boson of gravity, coupling to the energy-momentum tensor. Its superpartner, the gravitino $\tilde{G}$, is a spin $3 / 2$ particle that couples to the supercurrent $J_{Q}^{\mu}$, which generates supersymmetry. The gravitino plays an important role in the phenomenology of some supersymmetric models. 


\subsection{Supersymmetric Matter Interactions}

Given the particles of Table 1, we may then construct the most general supersymmetric Lagrangian. To construct the matter interactions, it is convenient to define the superpotential

$$
W=\frac{1}{2} \mu_{i j} \Psi_{i} \Psi_{j}+\frac{1}{3} y_{i j k} \Psi_{i} \Psi_{j} \Psi_{k},
$$

a gauge-invariant function of chiral superfields. The most general matter coupling is then compactly expressed in superfield notation as

$$
\mathcal{L}_{\text {matter }}=\left.\Psi_{i}^{\dagger} \Psi_{i}\right|_{\theta \theta \bar{\theta} \bar{\theta}}+\left[\left.W\right|_{\theta \theta}+\text { H.c. }\right],
$$

where one takes the components in the $\theta, \bar{\theta}$ expansion as indicated. The trilinear superpotential terms generate the Yukawa couplings of the standard model with dimensionless couplings $y_{i j k}$, along with other interactions related by supersymmetry. The parameters $\mu_{i j}$ have mass dimension 1 .

In component fields, Eq. (7) becomes

$$
\begin{aligned}
& \mathcal{L}_{\text {matter }}=\partial^{\mu} \phi_{i}^{*} \partial_{\mu} \phi_{i}+i \bar{\psi}_{i} \bar{\sigma}^{\mu} \partial_{\mu} \psi_{i}+F_{i}^{*} F_{i} \\
& \quad+\left[\mu_{i j}\left(\phi_{i} F_{j}-\frac{1}{2} \psi_{i} \psi_{j}\right)+y_{i j k}\left(\phi_{i} \phi_{j} F_{k}-\phi_{i} \psi_{j} \psi_{k}\right)+\text { H.c. }\right] .
\end{aligned}
$$

The first line includes canonical kinetic terms for every $\psi_{i}$ and $\phi_{i}$. As discussed above, however, there are no corresponding terms for the auxiliary fields $F_{i}$. These may therefore be removed by their equations of motion

$$
F_{i}^{*}=-\mu_{i j} \phi_{j}-y_{i j k} \phi_{j} \phi_{k} .
$$

With this substitution, the complete matter Lagrangian becomes

$$
\begin{gathered}
\mathcal{L}_{\text {matter }}=\partial^{\mu} \phi_{i}^{*} \partial_{\mu} \phi_{i}+i \bar{\psi}_{i} \bar{\sigma}^{\mu} \partial_{\mu} \psi_{i}-\frac{1}{2} \mu_{i j} \psi_{i} \psi_{j}-\frac{1}{2} \mu_{i j}^{*} \bar{\psi}_{i} \bar{\psi}_{j} \\
-y_{i j k} \phi_{i} \psi_{j} \psi_{k}-y_{i j k}^{*} \phi_{i}^{*} \bar{\psi}_{j} \bar{\psi}_{k}-\left|\mu_{i j} \phi_{j}+y_{i j k} \phi_{j} \phi_{k}\right|^{2} .
\end{gathered}
$$

Terms such as the last one in Eq. (10) are called F-terms, as they arise from $\left|F_{i}\right|^{2}$.

From Eq. (10) we find that both scalar and fermion masses are determined by $\mu$, and they are degenerate. This is a generic prediction of exact supersymmetry. The absence of a boson with the couplings and mass of the electron implies that supersymmetry must be broken. In addition, we see that there are trilinear and quartic scalar couplings. The quartic couplings are completely determined by the Yukawa couplings $y$. For this reason, the quadratically-divergent contributions to the Higgs boson mass shown in 

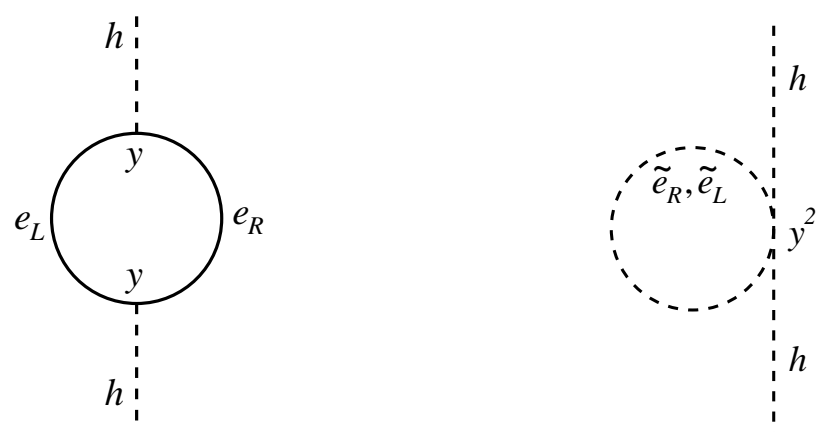

Fig. 1. Quadratically-divergent contributions to the Higgs boson mass in supersymmetry. These cancel exactly in the limit of exact supersymmetry.

Fig. 1 exactly cancel, as required for supersymmetry to stabilize the gauge hierarchy.

The superpotential of the MSSM is

$$
W=\epsilon_{a b}\left[-y_{i j}^{u} H_{u}^{a} Q_{i}^{b} U_{j}^{c}+y_{i j}^{d} H_{d}^{a} Q_{i}^{b} D_{j}^{c}+y_{i j}^{e} H_{d}^{a} L_{i}^{b} E_{j}^{c}-\mu H_{u}^{a} H_{d}^{b}\right],
$$

where $a, b$ are $\mathrm{SU}(2)$ indices contracted by the anti-symmetric $\epsilon$ tensor with $\epsilon_{12}=1$, and $i, j$ are generational indices. As in Table 1, the superscript $c$ denotes charge conjugation. Gauge invariance forbids bilinear terms in the standard model, but the additional Higgs doublet of the MSSM, $H_{u}$, may have bilinear couplings. Linear terms are forbidden by gauge invariance in the MSSM, but may be present in extensions of the MSSM that contain complete gauge singlets. Additional non-renormalizable couplings may also be included in extensions of the MSSM with quartic and higher order superpotential terms suppressed by some heavy mass scale.

Eq. (11) is not the most general gauge invariant superpotential. For example, as evident in Table 1, the superfields $L_{i}$ and $H_{d}$ have identical quantum numbers, leading to additional possible terms. The complete list of omitted terms is

$$
\begin{aligned}
W_{L} & =\lambda_{i j k} L_{i} L_{j} E_{k}^{c}+\mu_{i} L_{i} H_{u} \\
W_{L B} & =\lambda_{i j k}^{\prime} L_{i} Q_{j} D_{k}^{c} \\
W_{B} & =\lambda_{i j k}^{\prime \prime} U_{i}^{c} D_{j}^{c} D_{k}^{c} .
\end{aligned}
$$

$W_{L}$ violates lepton number $L, W_{B}$ violates baryon number $B$, and $W_{L B}$ violates both $L$ and $B$. If all terms are present, protons decay through renormalizable operators, and present bounds from proton decay are extremely 
stringent. All of these terms may be eliminated by assigning to each particle an $R$-parity $R=(-1)^{3(B-L)+2 S}$, where $S$ is spin, and requiring that $R$-parity be multiplicatively conserved. All standard model particles have $R=1$ and all superpartners have $R=-1$, and so all terms of Eq. (12) are eliminated. $R$-parity conservation implies that the lightest supersymmetric particle (LSP) is stable, with strong implications for collider studies and dark matter. We will focus on $R$-parity conserving supersymmetry throughout this review.

\subsection{Supersymmetric Gauge Interactions}

The interactions of the MSSM that involve gauge fields fall into several categories. The terms involving only gauge fields are

$$
-\frac{1}{4} F_{\mu \nu}^{a} F^{a \mu \nu}+i \bar{\lambda}^{a} \bar{\sigma}^{\mu} \partial_{\mu} \lambda^{a}+\frac{1}{2} D^{a 2}
$$

for each gauge group, where a labels gauge group generators. The first two terms are the canonical kinetic terms for gauge bosons and gauginos. As with the $F$ field, $D$ has no kinetic term and may be eliminated by its equation of motion. This leads to the quartic scalar couplings

$$
V_{D}=\frac{g_{s}^{2}}{2}\left[\sum_{a, i j} \phi_{i}^{\dagger} T_{3, i j}^{a} \phi_{j}\right]^{2}+\frac{g^{2}}{2}\left[\sum_{a, i j} \phi_{i}^{\dagger} T_{2, i j}^{a} \phi_{j}\right]^{2}+\frac{g^{\prime 2}}{2}\left[\sum_{i} \phi_{i}^{\dagger} Y_{i} \phi_{i}\right]^{2}
$$

where $T_{3}$ and $T_{2}$ are the generators of $\mathrm{SU}(3)$ and $\mathrm{SU}(2)$, and $Y$ is hypercharge. These are known as D-terms. As in Sec. (2.2), these quartic interactions are completely determined by standard model couplings - in this case, the gauge couplings. A host of quadratically-divergent contributions to the Higgs mass that involve gauge interactions are thereby exactly canceled by supersymmetric contributions, allowing supersymmetry to completely stabilize the gauge hierarchy.

The gauge-matter couplings include $v_{\mu} \phi \partial^{\mu} \phi^{*}$ couplings, as well as four-point $v^{\mu} v_{\mu} \phi \phi^{*}$ interactions, where $v^{\mu}$ denotes gauge bosons. Finally, and perhaps of greatest significance for linear collider studies, there are sfermion-fermion-gaugino couplings, the supersymmetric analogues to the fermion-fermion-gauge boson couplings. These are of the form

$$
\sqrt{2} g \phi_{i} T_{i j}^{a} \psi_{j} \lambda^{a}+\text { H.c. , }
$$

where the coupling $g$ is a standard model gauge coupling. Supersymmetry relates the couplings of gauginos to the couplings of gauge bosons. As we will discuss in Sec. (7), this fact may be exploited to definitively identify 
new particles as superpartners, as well as to determine the mass scales of undiscovered superpartners.

\subsection{Supersymmetry-breaking Terms}

So far we have been discussing the purely supersymmetric part of the MSSM Lagrangian. However, as noted above, supersymmetry predicts that superpartners have equal mass. This is also evident from the supersymmetry algebra: the supersymmetry generator commutes with the momentum operator, and so

$$
(H Q-Q H)|\phi\rangle=\left(E_{\psi}-E_{\phi}\right)|\psi\rangle=0 .
$$

Because we have not found superpartners degenerate with the known particles, supersymmetry must be broken.

Supersymmetry may be broken in many ways. However, to preserve the strong phenomenological motivation of solving the gauge hierarchy problem, it is imperative that the quadratic divergences, so elegantly removed by exact supersymmetry, are not re-introduced. When we require this, the allowed terms, known as soft supersymmetry-breaking terms, are limited to four classes: gaugino masses $M \lambda \lambda$, scalar masses $m^{2} \phi^{*} \phi$, trilinear scalar couplings $A \phi \phi \phi$ (A-terms), and bilinear scalar couplings $B \phi \phi(B$-terms). No new dimensionless couplings are introduced, preserving the cancellations discussed in Secs. 2.2 and 2.3. Also note, however, that not all dimensionful contributions are allowed; for example, terms of the form $\phi_{i}^{*} \phi_{j} \phi_{k}$ are not soft.

In the MSSM, the full set of soft terms is

$$
\begin{aligned}
& -\mathcal{L}_{\text {soft }}=\frac{1}{2}\left(M_{1} \tilde{B} \tilde{B}+M_{2} \tilde{W}^{a} \tilde{W}^{a}+M_{3} \tilde{g}^{a} \tilde{g}^{a}\right) \\
& +m_{Q i j}^{2}\left(\tilde{u}_{L i}^{*} \tilde{u}_{L j}+\tilde{d}_{L i}^{*} \tilde{d}_{L j}\right)+m_{U i j}^{2} \tilde{u}_{R i}^{*} \tilde{u}_{R j}+m_{D i j}^{2} \tilde{d}_{R i}^{*} \tilde{d}_{R j} \\
& +m_{L i j}^{2}\left(\tilde{\nu}_{L i}^{*} \tilde{\nu}_{L j}+\tilde{e}_{L i}^{*} \tilde{e}_{L j}\right)+m_{E i j}^{2} \tilde{e}_{R i}^{*} \tilde{e}_{R j} \\
& +\epsilon_{a b}\left[-\tilde{A}_{i j}^{u} H_{u}^{a} \tilde{q}_{i}^{b} \tilde{u}_{R j}^{*}+\tilde{A}_{i j}^{d} H_{d}^{a} \tilde{q}_{i}^{b} \tilde{d}_{R j}^{*}+\tilde{A}_{i j}^{e} H_{d}^{a} \tilde{l}_{i}^{b} \tilde{e}_{R j}^{*}\right. \\
& \left.\quad-B H_{u}^{a} H_{d}^{b}+\text { H.c. }\right] .
\end{aligned}
$$

Gauge invariance requires that scalar particles in the same electroweak doublet receive the same soft masses. The $A$ - and $B$-terms mimic the Yukawa and $\mu$ terms of the superpotential. If $R$-parity is broken, there are additional soft terms corresponding to the superpotential terms of Eq. 12.

What is the origin of these soft terms? At first sight, supersymmetry has just introduced a bewildering array of new parameters. If supersymmetry is to take us closer to a fundamental theory, we would hope that these 
parameters are not completely arbitrary. In fact, however, blind hope is not required! Experimental constraints already guarantee that, if weak-scale supersymmetry exists, the soft terms cannot be generic. These constraints, some of which will be reviewed in Sec. (3), imply that some pattern must emerge. Further, we will see in Sec. (何) that the soft terms contain an imprint of physics at even more microscopic scales, and their determination may shed light on energy scales as high as the Planck scale. Just as discovering the mechanism of spontaneous gauge symmetry breaking is one of the key issues in standard model physics, uncovering the mechanism of supersymmetry breaking is the central issue of supersymmetric physics. The promise of model-independent measurements of the soft parameters is the prime supersymmetric motivation for the linear collider.

\subsection{Sleptons}

Given both the supersymmetric and the soft supersymmetry-breaking terms, we are now ready to determine the mass eigenstates of the MSSM. For lack of space, we focus on those with electroweak quantum numbers only, although squarks and gluinos may also be produced at linear colliders with many interesting results (see, for example, Refs. 112, 13, 14, 15, 16, 17]).

We first discuss the scalar superpartners. There are six charged slepton states. These receive masses from soft terms and also, after electroweak symmetry breaking, from Yukawa couplings, $F$-terms, and $D$-terms. The full set of slepton mass terms is $\tilde{l}^{\dagger} \mathcal{M}_{\tilde{l}}^{2} \tilde{l}$, where $\tilde{l}^{T}=\left(\tilde{e}_{L}, \tilde{\mu}_{L}, \tilde{\tau}_{L}, \tilde{e}_{R}, \tilde{\mu}_{R}, \tilde{\tau}_{R}\right)$, and the $6 \times 6$ mass matrix is

$$
\mathcal{M}_{\tilde{l}}^{2}=\left(\begin{array}{cc}
m_{L L}^{2} & m_{L R}^{2} \\
m_{L R}^{2 \dagger} & m_{R R}^{2}
\end{array}\right) .
$$

The diagonal $3 \times 3$ blocks are

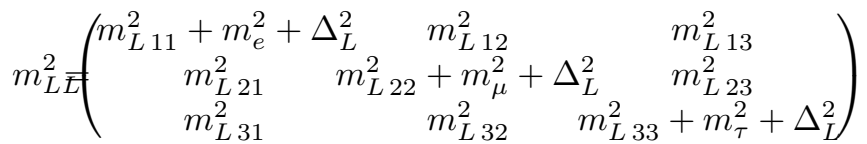

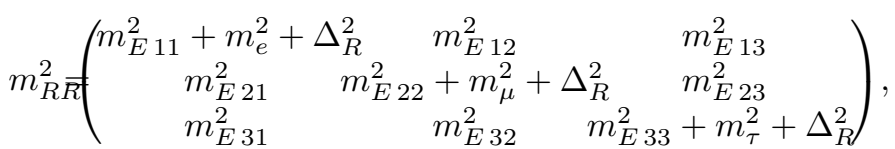

where $\Delta_{L}^{2}=m_{Z}^{2}\left(-\frac{1}{2}+\sin ^{2} \theta_{W}\right) \cos 2 \beta$ and $\Delta_{R}^{2}=m_{Z}^{2}\left(-\sin ^{2} \theta_{W}\right) \cos 2 \beta$. The $\Delta_{L, R}^{2}$ contributions originate from gauge interactions, the $D$-terms 
of Eq. (14), and are therefore flavor-diagonal. While they contain no supersymmetry-breaking parameters, they require electroweak symmetry breaking, which in turn relies on supersymmetry-breaking soft masses for the Higgs scalars. The off-diagonal blocks are given by

$$
m_{L R}^{2}=-\left(\begin{array}{l}
\tilde{A}_{11}^{e} v_{d}+\mu y_{11}^{e} v_{u} \tilde{A}_{12}^{e} v_{d}+\mu y_{12}^{e} v_{u} \tilde{A}_{13}^{e} v_{d}+\mu y_{13}^{e} v_{u} \\
\tilde{A}_{21}^{e} v_{d}+\mu y_{21}^{e} v_{u} \tilde{A}_{22}^{e} v_{d}+\mu y_{22}^{e} v_{u} \tilde{A}_{23}^{e} v_{d}+\mu y_{23}^{e} v_{u} \\
\tilde{A}_{31}^{e} v_{d}+\mu y_{31}^{e} v_{u} \tilde{A}_{32}^{e} v_{d}+\mu y_{32}^{e} v_{u} \tilde{A}_{33}^{e} v_{d}+\mu y_{33}^{e} v_{u}
\end{array}\right)
$$

Finally, the sneutrino masses are $\tilde{\nu}^{\dagger} \mathcal{M}_{\tilde{\nu}} \tilde{\nu}$, where $\tilde{\nu}^{T}=\left(\tilde{\nu}_{e}, \tilde{\nu}_{\mu}, \tilde{\nu}_{\tau}\right)$, and

$$
\mathcal{M}_{\tilde{\nu}}^{2}=\left(\begin{array}{ccc}
m_{L 11}^{2}+\Delta_{\nu}^{2} & m_{L 12}^{2} & m_{L 13}^{2} \\
m_{L 21}^{2} & m_{L 22}^{2}+\Delta_{\nu}^{2} & m_{L 23}^{2} \\
m_{L 31}^{2} & m_{L 32}^{2} & m_{L 33}^{2}+\Delta_{\nu}^{2}
\end{array}\right)
$$

where $\Delta_{\nu}^{2}=m_{Z}^{2} \frac{1}{2} \cos 2 \beta$.

In general, we see that the sleptons masses are complicated. Note that, while it is always possible to rotate to a basis where the Yukawa couplings $y^{e}$ are diagonal, there is no reason for these rotations to simultaneously diagonalize the soft term matrices $m_{L}^{2}, m_{E}^{2}$, and $\tilde{A}^{e}$. Studies of slepton flavor mixing may shed light on fundamental issues, such as the masses of standard model quarks and leptons, and will be discussed in Sec. (5.4).

Ultimately one hopes to study the sleptons in full generality at colliders. However, in studies so far, simplifying assumptions are typically made to reduce the problem to a more manageable form. A common assumption is flavor conservation, so that $y^{e}, m_{L}^{2}, m_{E}^{2}$, and $\tilde{A}^{e}$ are all simultaneously diagonalizable. The $6 \times 6$ matrix then reduces to three $2 \times 2$ blocks of the form

$$
m_{\tilde{\tau}}^{2}=\left(\begin{array}{cc}
m_{L 33}^{2}+m_{\tau}^{2}+\Delta_{L}^{2} & -m_{\tau}\left(A_{\tau}+\mu \tan \beta\right) \\
-m_{\tau}\left(A_{\tau}+\mu \tan \beta\right) & m_{E 33}^{2}+m_{\tau}^{2}+\Delta_{R}^{2}
\end{array}\right)
$$

and similarly for the first and second generations. The $\tilde{A}^{e}$ parameters have been replaced by $A$ parameters, following convention. A common assumption is that $A_{e}, A_{\mu}$, and $A_{\tau}$ are of the same order. This is motivated by some forms of supersymmetry breaking, but is by no means a universal requirement. With this assumption, bounds on $\left|A_{\tau}\right|$ from the requirement that there be no tachyonic staus typically imply that left-right mixing in selectrons and smuons is insignificant. However, left-right mixing may be important for the staus. In fact, although suppressed by $m_{\tau}$, the factor $\mu \tan \beta$ leads to large mixing in many models. In general, the stau mass 
eigenstates and eigenvalues are

$$
\begin{aligned}
& \tilde{\tau}_{1}=\tilde{\tau}_{L} \cos \theta_{\tau}+\tilde{\tau}_{R} \sin \theta_{\tau} \\
& \tilde{\tau}_{2}=-\tilde{\tau}_{L} \sin \theta_{\tau}+\tilde{\tau}_{R} \cos \theta_{\tau}
\end{aligned}
$$

and

$$
m_{\tilde{\tau}_{1}, \tilde{\tau}_{2}}^{2}=\frac{1}{2}\left[m_{\tilde{\tau} L L}^{2}+m_{\tilde{\tau} R R}^{2} \mp \sqrt{\left(m_{\tilde{\tau} L L}^{2}-m_{\tilde{\tau} R R}^{2}\right)^{2}+4\left(m_{\tilde{\tau} L R}^{2}\right)^{2}}\right] .
$$

As we will see, when the slepton masses are assumed to be unified at a high scale, renormalization group effects imply that staus are the lightest sleptons and so are of great importance in collider studies.

Finally, in the flavor conserving case, Eqs. (19) and (21) imply

$$
m_{\tilde{e}_{L}}^{2}-m_{\tilde{\nu}_{e}}^{2}=-m_{W}^{2} \cos 2 \beta .
$$

This mass relation is an extremely robust prediction, resulting only from gauge symmetry and supersymmetry in the MSSM. It therefore provides a non-trivial test that newly-discovered scalars must pass to be identified as left-handed sleptons.

\subsection{Charginos and Neutralinos}

We now turn to the fermionic superpartners. The charged fermions are Winos and Higgsinos. Without electroweak breaking, the Winos have soft mass $M_{2}$ from Eq. (17), and the Higgsinos get mass through the superpotential term $-\mu H_{u} H_{d}$ of Eq. (11). Once electroweak symmetry is broken, however, the Higgs-Wino-Higgsino interactions of Eq. (15) generate Wino-Higgsino mixing. In the standard model, proper electroweak symmetry breaking requires a Higgs vacuum expectation value (vev) $\langle h\rangle^{2}=v^{2} / 2$, where $\left(g^{2}+g^{\prime 2}\right) v^{2} / 2=m_{Z}^{2}$. As noted above, however, the MSSM is a two Higgs doublet model, and so the vev $v$ may be shared between both neutral Higgses: $\left\langle H_{u}^{0}\right\rangle^{2}+\left\langle H_{d}^{0}\right\rangle^{2}=v^{2} / 2$. This freedom is typically parametrized by $\tan \beta$, where

$$
\left\langle H_{u}^{0}\right\rangle=\frac{v}{\sqrt{2}} \sin \beta, \quad\left\langle H_{d}^{0}\right\rangle=\frac{v}{\sqrt{2}} \cos \beta .
$$

With this parametrization, the charged mass terms are $\psi^{-{ }^{T}} \mathcal{M}_{C} \psi^{+}+$H.c., where $\psi^{ \pm T}=\left(-i \tilde{W}^{ \pm}, \tilde{H}^{ \pm}\right)$and

$$
\mathcal{M}_{C}=\left(\begin{array}{cc}
M_{2} & \sqrt{2} m_{W} \sin \beta \\
\sqrt{2} m_{W} \cos \beta & \mu
\end{array}\right) .
$$


$\mathcal{M}_{C}$ may be diagonalized by unitary matrices $U$ and $V$ through $M_{D}=$ $U^{*} \mathcal{M}_{C} V^{-1}$. If $\mathcal{M}_{C}$ is real, $U$ and $V$ may also be chosen real and may be parametrized as

$$
U=\left(\begin{array}{cc}
\cos \phi_{-} & \sin \phi_{-} \\
-\sin \phi_{-} & \cos \phi_{-}
\end{array}\right), \quad V=\left(\begin{array}{cc}
\cos \phi_{+} & \sin \phi_{+} \\
-\sin \phi_{+} & \cos \phi_{+}
\end{array}\right) .
$$

The resulting mass eigenstates are called charginos and are $\tilde{\chi}_{i}^{-}=U_{i j} \psi_{j}^{-}$ and $\tilde{\chi}_{i}^{+}=V_{i j} \psi_{j}^{+}$. In the limit where $M_{2},|\mu| \gg m_{W}$ the mass eigenstates become nearly pure Winos and Higgsinos. 9 The mixing angles are sensitive to $\tan \beta$ when $\tan \beta \sim 1$.

The neutral fermionic superpartners are the Bino, the neutral Wino, and the two neutral Higgsinos. As with charginos, electroweak symmetry breaking generates gaugino-Higgsino mixing. The resulting mass eigenstates are called neutralinos. The neutralino mass matrix is $\frac{1}{2} \psi^{0^{T}} \mathcal{M}_{N} \psi^{0}+$ H.c., where $\psi^{0^{T}}=\left(-i \tilde{B},-i \tilde{W}^{3}, \tilde{H}_{d}^{0}, \tilde{H}_{u}^{0}\right)$ and

$$
\mathcal{M}_{N}=\left(\begin{array}{cccc}
M_{1} & 0 & -m_{Z} c_{\beta} s_{W} & m_{Z} s_{\beta} s_{W} \\
0 & M_{2} & m_{Z} c_{\beta} c_{W} & -m_{Z} s_{\beta} c_{W} \\
-m_{Z} c_{\beta} s_{W} & m_{Z} c_{\beta} c_{W} & 0 & -\mu \\
m_{Z} s_{\beta} s_{W} & -m_{Z} s_{\beta} c_{W} & -\mu & 0
\end{array}\right),
$$

and we have introduced the shorthand notation $s_{W} \equiv \sin \theta_{W}, c_{W} \equiv \cos \theta_{W}$, $s_{\beta} \equiv \sin \beta$, and $c_{\beta} \equiv \cos \beta$. The neutralino mass eigenstates are $\tilde{\chi}_{i}^{0}=N_{i j} \psi_{j}^{0}$, where $N$ diagonalizes $\mathcal{M}_{N}$.

In order of increasing mass, the two charginos are labeled $\tilde{\chi}_{1}^{ \pm}$and $\tilde{\chi}_{2}^{ \pm}$, and the four neutralinos are $\tilde{\chi}_{1}^{0}, \tilde{\chi}_{2}^{0}, \tilde{\chi}_{3}^{0}$, and $\tilde{\chi}_{4}^{0}$. There are several phenomenologically interesting limits for the chargino and neutralino mass spectrum:

- $M_{1}<M_{2}<|\mu|$ : "Bino LSP scenarios." As we will discuss in Sec. (4.1), this case is realized in much, but not all, of minimal supergravity parameter space. The lightest state is $\tilde{\chi}_{1}^{0} \approx \tilde{B}$, followed by a nearly degenerate triplet, $\tilde{\chi}_{2}^{0} \approx \tilde{W}^{0}$ and $\tilde{\chi}_{1}^{ \pm} \approx \tilde{W}^{ \pm}$. There is typically a large spacing between the lightest state and all others.

- $|\mu|<M_{1}, M_{2}$ : "Higgsino LSP scenarios." $\tilde{\chi}_{1}^{0}, \tilde{\chi}_{2}^{0}, \tilde{\chi}_{1}^{+}$are all fairly degenerate in mass and Higgsino-like. Radiative corrections are important in determining the mass differences.

$\overline{{ }^{a} \text { We assume that gaugino masses }} M_{i}$ are positive and real, unless otherwise stated. 
- $M_{2}<M_{1},|\mu|$ : "Wino LSP scenarios." These are realized in a variety of models, including some anomaly-mediation models. (See Sec. (4.5).) $\tilde{\chi}_{1}^{0}$ and $\tilde{\chi}_{1}^{ \pm}$are Wino-like and extremely mass degenerate, and the charginos may have an observable lifetime before decaying to the neutralino. In this case, radiative corrections are essential in determining the mass difference.

\section{Successes and Puzzles}

The discovery of weak-scale supersymmetry will be accompanied by many fundamental questions, such as those listed in Sec. (1). Here we expand upon a number of these, both to highlight some important goals of the linear collider program and also to motivate the array of models to be described in Sec. (雨).

\subsection{Unification}

The unification of forces requires that gauge couplings unify at some scale. The renormalization group evolutions of gauge couplings in the standard model and the MSSM are shown in Fig. 2. For the MSSM, all sparticles are assumed to lie between $250 \mathrm{GeV}$ and $1 \mathrm{TeV}$. In the standard model, the gauge couplings obviously do not unify. In the MSSM, however, they unify at the $1 \%$ level. This is widely regarded as the strongest quantitative motivation for any framework for physics beyond the standard model proposed to date.

The fact that the three couplings meet in supersymmetry is significant, but so is where they meet. The couplings unify with value $\alpha_{\text {unif }} \approx 1 / 24$ at $M_{\mathrm{GUT}} \simeq 2 \times 10^{16} \mathrm{GeV}$. If the couplings had unified at $\alpha \gtrsim 1$, unification would occur beyond the perturbative regime. At the same time, unification at scales above $M_{\mathrm{Pl}} \simeq 10^{19} \mathrm{GeV}$ would be invalidated by quantum gravity effects, while unification at scales $Q \lesssim 10^{16} \mathrm{GeV}$ would imply proton decay at levels in conflict with experiment. (See Sec. (3.5).) In fact, the proximity of $M_{\mathrm{GUT}}$ to $M_{\mathrm{Pl}}$ gives hope that the effective gravitational coupling, $\alpha_{\mathrm{grav}} \sim$ $E^{2} / M_{\mathrm{Pl}}^{2}$ also unifies with the standard model gauge couplings near $Q \sim$ $M_{\mathrm{Pl}}$, as is motivated in string theory. The discrepancy between $M_{\mathrm{GUT}}$ and $M_{\mathrm{Pl}}$ may be resolved by a variety of mechanisms [18, 19].

The unification of gauge couplings has immediate implications for the linear collider. Simple gauge group unification requires also gaugino mass unification. The quantities $M_{i} / g_{i}^{2}$ are invariants of one-loop renormalization group evolution. (Two-loop effects typically lead to small deviations [6].) If 


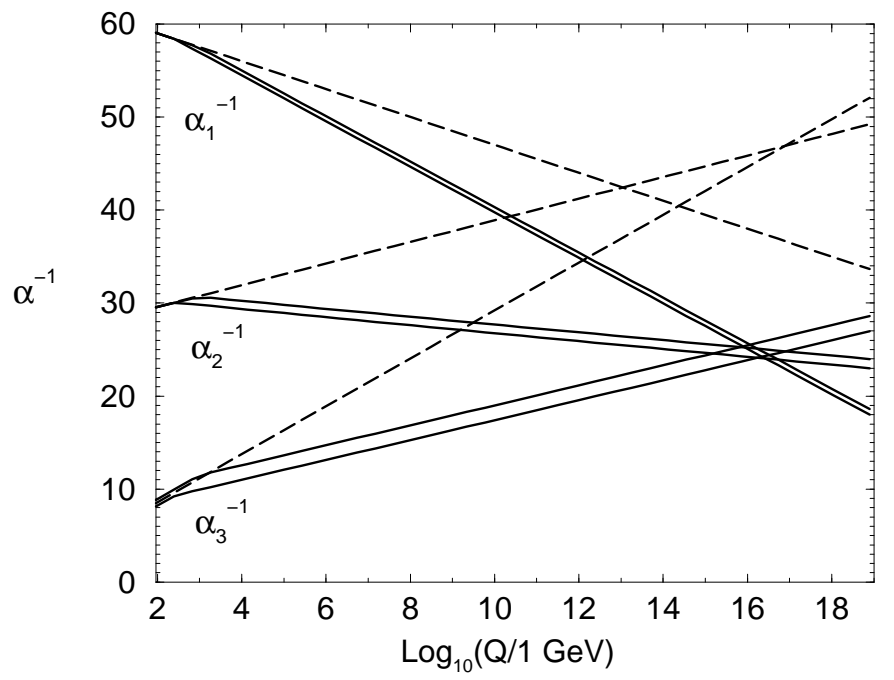

Fig. 2. The renormalization group evolution of the three gauge coupling constants in the standard model (dashed) and in the MSSM (solid) [6].

all gauge couplings and gaugino masses are unified at any high scale, one therefore expects

$$
M_{1}: M_{2}: M_{3}=g_{1}^{2}: g_{2}^{2}: g_{3}^{2} \approx 1: 2: 7
$$

at lower scales, where $g_{1}, g_{2}$, and $g_{3}$ are the hypercharge, weak, and strong couplings. The model-independent measurement of gaugino mass parameters is then of great importance, and the verification of $2 M_{1} \approx M_{2}$ would provide strong supporting evidence for unification.

Gauge group unification also implies that the particle content of the MSSM lies in representations of a larger gauge group. There is already tantalizing evidence for this - although the quantum numbers of the standard model particles shown in Table 1 appear rather arbitrary, they are consistent with their placement in $\overline{\mathbf{5}}+\mathbf{1 0}$ representations of $\mathrm{SU}(5)$, or, with the addition of a right-handed neutrino, in the spinor representation 16 of $\mathrm{SO}(10)$. These unifications imply relations between Yukawa couplings and soft supersymmetry-breaking terms of particles in the same grand unified theory (GUT) multiplets, providing additional motivations for modelindependent measurements. 


\subsection{Dark Matter}

As noted in Sec. (2.2), the most general gauge-invariant supersymmetric standard model allows proton decay through renormalizable operators, in gross violation of proton lifetime limits. $R$-parity conservation is one elegant way to prevent this. This has the immediate consequence that the lightest supersymmetric particle (LSP) is stable. In many models, this LSP is a neutralino, a weakly-interacting, weak-scale mass particle that is a natural candidate for non-baryonic cold dark matter [20, 21, 22, 23].

If supersymmetry is discovered at colliders, studies of the superpartner spectrum will shed light on this hypothesis. Of course, collider experiments will never be able to prove that the LSP is dark matter - this requires knowing that the LSP is stable on cosmological time scales. However, if the LSP is found to be charged, colored, or unstable, supersymmetric dark matter will be largely disfavored. On the other hand, if the LSP is found to be a seemingly stable neutralino, there will be much work to do. One interesting test will be to determine the neutralino's thermal relic density. The neutralino's relic abundance is determined by its annihilation cross sections through processes such as those of Fig. 3. The processes $\chi \chi \rightarrow f \bar{f}$ are chirality suppressed, and so the most relevant $t$-channel sfermions are typically stops, sbottoms, and staus. If the neutralino's mass and composition are known, as well as the masses and compositions of these sfermions, its thermal relic density may be accurately computed, assuming a standard thermal history for the universe. Values near the favored dark matter density of $\Omega \approx 0.3$ will be strong evidence in favor of neutralino dark matter. Collider and conventional dark matter experiments will also play complementary roles. The interactions of neutralinos with matter are given by interactions such as those in Fig. 4 . By determining the relevant supersymmetry parameters, collider experiments may guide dark matter searches, or confirm their signals as being due to supersymmetry. We will discuss the relation of linear collider experiments to cosmology in more detail in Sec. (10).

\subsection{Flavor Violation}

Weak-scale supersymmetry generically induces gross violations of constraints on flavor-violating observables [24]. Among the best known is the supersymmetric contribution to the $K_{L}-K_{S}$ mass splitting. Supersymmetry contributes to this through box diagrams, such as the one involving squarks and gluinos shown in Fig. 5 . The crosses on the squark propaga- 


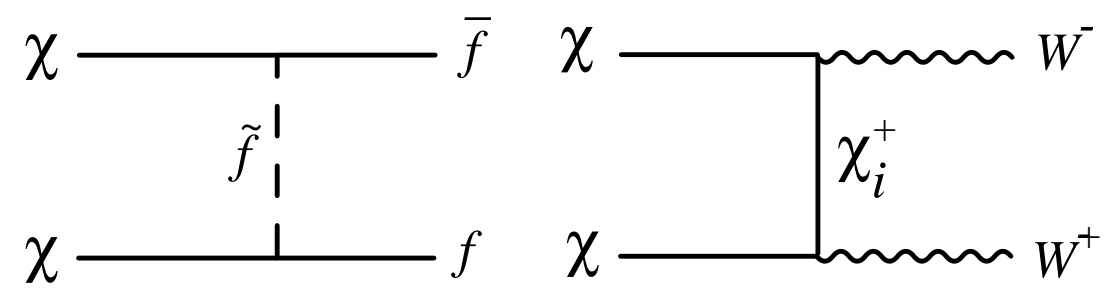

Fig. 3. Neutralino dark matter annihilation processes.
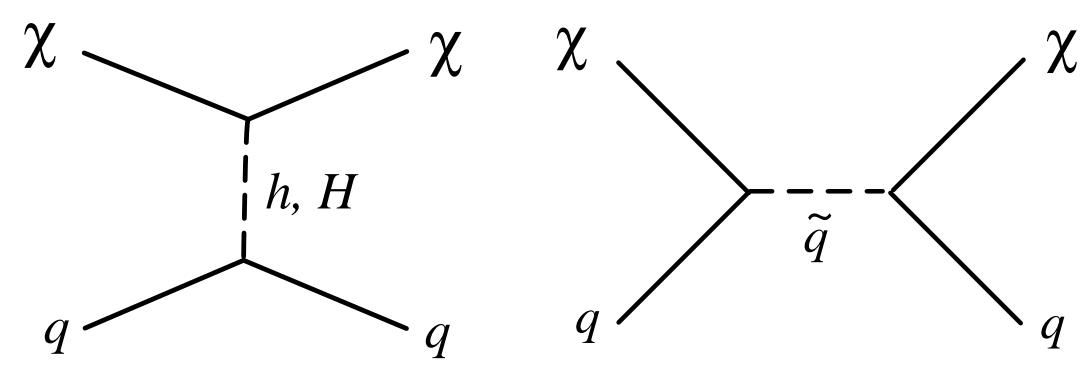

Fig. 4. Neutralino dark matter scattering processes.

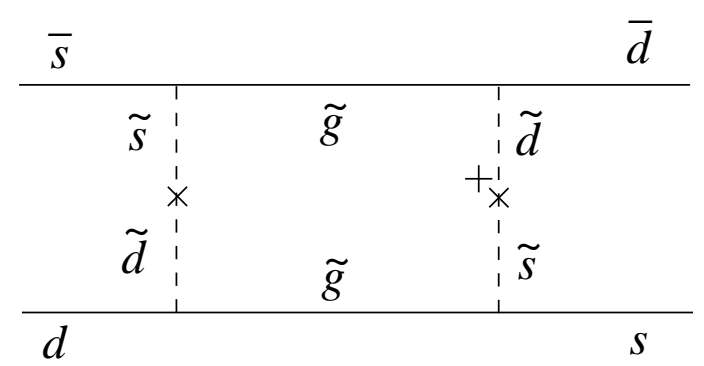

Fig. 5. Supersymmetric contribution to the $K_{L}-K_{S}$ mass splitting.

tors are flavor-violating mass insertions $m_{\tilde{q}_{12}}^{2}$. As noted in Sec. (2.5), there is no reason a priori to assume that squark and quark mass matrices are simultaneously diagonalizable, and so such flavor-violating mass insertions generically exist. 
The bound from the measured $\Delta m_{K}$ implies [25]

$$
\left[\frac{10 \mathrm{TeV}}{m_{\tilde{q}, \tilde{g}}}\right]^{2}\left[\frac{\Delta m_{\tilde{q}_{12}}^{2} / m_{\tilde{q}}^{2}}{0.1}\right]^{2} \lesssim 1
$$

For TeV-scale squarks and gluinos, the flavor-violating parameters must be very small so that the supersymmetric generalization of the GIM mechanism applies. Alternatively, for $\mathcal{O}(1)$ flavor mixing, squark and gluino masses must be of order $10 \mathrm{TeV}$, well above the weak scale.

The supersymmetric flavor problem is among the most pressing phenomenological problems of supersymmetry, and motivates many models that naturally produce degenerate squarks, very heavy squarks and gluinos, or quark-squark alignment. Note that this is not simply a problem in the hadronic sector - bounds on lepton flavor violation (LFV) from $\mu-e$ conversion, $\mu \rightarrow e \gamma$, and other processes, cause similar difficulties. If supersymmetry is discovered, one immediate question will be how the supersymmetric flavor puzzle is resolved.

\subsection{CP Violation}

Weak-scale supersymmetry also generically violates bounds on $\mathrm{CP}$ violation. Bounds from $\epsilon_{K}$ are numerically the most stringent, but are typically satisfied in models that eliminate flavor violation in some natural way. However, CP-violating, but flavor-conserving, constraints remain a problem. A prime example of these constraints is the electron's electric dipole moment (EDM). The supersymmetric contribution to this observable is shown in Fig. 6. This contribution requires 26

$$
\left[\frac{2 \mathrm{TeV}}{m_{\tilde{e}}}\right]^{2}\left[\frac{\mu M_{1}}{m_{\tilde{e}}^{2}}\right] \tan \beta \sin \phi_{C P} \lesssim 1,
$$

that is, uncomfortably heavy selectrons or small CP-violating phases $\phi_{C P} \ll 1$. As in the flavor-violating case, this problem is not confined to one sector - a similarly stringent constraint in the hadronic sector follows from the neutron EDM. Again, the discovery of supersymmetry will raise the question of how these supersymmetric CP problems are solved.

\subsection{Proton Decay}

In supersymmetric theories, proton decay through renormalizable operators may be eliminated by $R$-parity conservation. However, in grand unified 


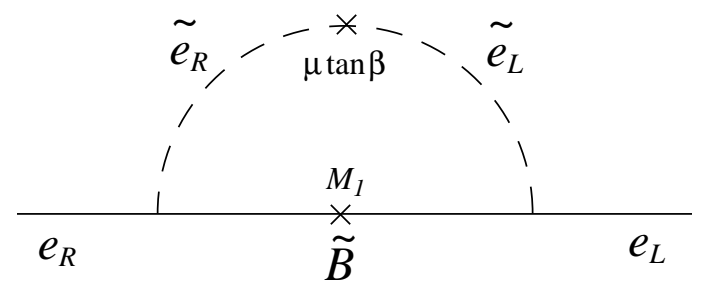

Fig. 6. Supersymmetric contribution to the electron's electric dipole moment. A photon attached to the charged internal line is implicit.

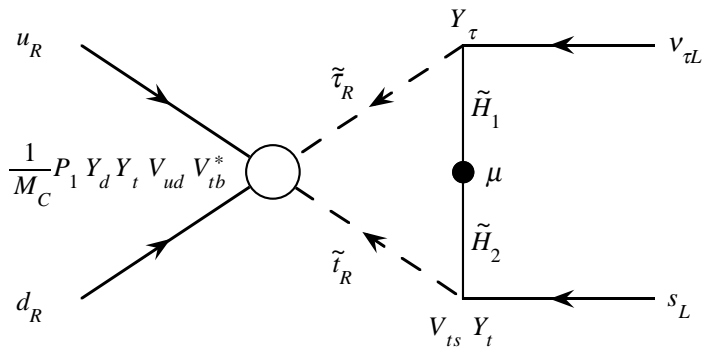

Fig. 7. Contribution to proton decay in supersymmetric grand unified theories 29].

theories (GUTs), proton decay is again allowed through non-renormalizable operators suppressed by the GUT scale.

The leading contribution for large $\tan \beta$ in supersymmetric $\mathrm{SU}(5)$ the-

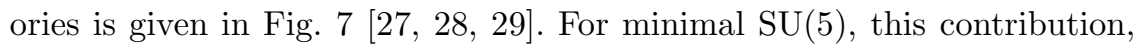
normalized to the current bounds from SuperKamiokande [30], implies

$$
\left[\frac{\tau\left(p \rightarrow K^{+} \bar{\nu}\right)}{1.6 \times 10^{33} \mathrm{yr}}\right]\left[\frac{(5 \mathrm{TeV})^{2} \mu^{2}}{m_{\tilde{q}}^{4}}\right]\left[\frac{\tan \beta}{4}\right]^{4}\left[\frac{2 \times 10^{16} \mathrm{GeV}}{M_{C}}\right]^{2} \lesssim 1
$$

where $M_{C}$ is the colored Higgs mass, typically expected to be of the order of the unification scale $M_{\mathrm{GUT}}$. This operator relies only on standard model flavor violation, and so is present even in supersymmetric theories with degenerate scalars and CP conservation.

The absence of observed proton decay is becoming a severe constraint for simple supersymmetric GUTs. It motivates models with naturally heavy superpartners, extended GUT models, or even those with some mechanism to unify gauge coupling without GUT gauge groups. The discovery of light squarks will immediately exclude the simplest GUT models. 


\section{Models}

If weak-scale supersymmetry is found in nature, the promise of linear colliders lies in the possibility of disentangling supersymmetry without modeldependent assumptions. However, without actual data, studies of the potential of colliders require some model framework to consider. The models studied are typically motivated by the desire to solve the problems without sacrificing the successes of Sec. (3). Here we describe several frameworks with distinct collider phenomenology.

For collider phenomenology, models are largely specified by their soft supersymmetry-breaking terms. What is the origin of the soft terms? It is typically expected that they are generated by some dynamical mechanism closely analogous to the mechanism of spontaneous gauge symmetry breaking. In electroweak symmetry breaking, for example, gauge symmetry is broken when the Higgs field gets a non-vanishing vev. For supersymmetry breaking, the $F$ fields play the role of the Higgs field. To see this, note that supersymmetry transformations on chiral superfields may be defined as $\delta_{\xi} \Psi=[\xi Q+\bar{\xi} \bar{Q}, \Psi]$. Expanding both sides in powers of $\theta$, we find

$$
\begin{aligned}
\delta_{\xi} \phi & =\sqrt{2} \xi \psi \\
\delta_{\xi} \psi & =-i \sqrt{2} \sigma^{\mu} \bar{\xi} \partial_{\mu} \phi+\sqrt{2} \xi F \\
\delta_{\xi} F & =-i \sqrt{2} \bar{\xi} \bar{\sigma}^{\mu} \partial_{\mu} \psi .
\end{aligned}
$$

If $F$ obtains a vev,

$$
\langle 0|F| 0\rangle=\langle 0|[\xi Q+\bar{\xi} \bar{Q}, \psi]| 0\rangle \neq 0
$$

implying that $Q|0\rangle \neq 0$, and supersymmetry is broken in the vacuum.

In most supersymmetric models, including those to be discussed below, the terms of the Lagrangian may be divided into three sectors: the supersymmetry breaking sector, containing only non-MSSM fields $Z$, the mediation sector coupling the $Z$ and MSSM fields, and the MSSM sector, containing only MSSM fields. Supersymmetry is broken when dynamical effects generate a vev for the $F$ component of a $Z$ field. This vev is called the scale of supersymmetry breaking. Terms in the mediation sector then generate soft terms for MSSM fields. For example, when $F_{Z}$ gets a vev, the soft scalar masses and trilinear $A$-terms of Eq. (17) will be generated by higher-dimensional operators suppressed by the mass scale $M$ of the 
mediation sector:

$$
\begin{aligned}
\left.\lambda_{i j} \frac{Z^{\dagger} Z}{M^{2}} \Psi_{i}^{\dagger} \Psi_{j}\right|_{\theta \theta} & \rightarrow \lambda_{i j} \frac{\left|\left\langle F_{Z}\right\rangle\right|^{2}}{M^{2}} \phi_{i}^{*} \phi_{j} \\
\left.\lambda_{i j k} \frac{Z}{M} \Psi_{i} \Psi_{j} \Psi_{k}\right|_{\theta \theta} & \rightarrow \lambda_{i j k} \frac{\left\langle F_{Z}\right\rangle}{M} \phi_{i} \phi_{j} \phi_{k} .
\end{aligned}
$$

Gaugino masses and $B$-terms may also be generated in this way. The sparticle masses and other weak-scale parameters therefore contains an imprint of terms in the mediation sector which are determined by physics at energies much higher than the weak scale. Field condensation does not alter the high energy behavior of the theory, and terms generated from $F$ condensation, such as those above, do not disturb the cancellation of quadratic divergences.

For most phenomenological applications, how supersymmetry is broken is largely irrelevant. Far more important is the supersymmetrymediation mechanism, that is, the terms of the mediation sector, and the supersymmetry-breaking scale. Both are crucial in determining the signatures of supersymmetry. The mediation terms determine the pattern of soft terms and, consequently, the masses, mixings, and interactions of the standard model superpartners. The supersymmetry-breaking scale determines the properties of the gravitino. The gravitino's mass is

$$
m_{\tilde{G}}=\frac{F_{\mathrm{DSB}}}{\sqrt{3} M_{\mathrm{Pl}}}
$$

and its interactions are given by

$$
\mathcal{L} \supset \frac{1}{F_{\mathrm{DSB}}}\left[m_{\tilde{f}}^{2} \bar{f} \tilde{f}+\frac{m_{\lambda}}{4 \sqrt{2}} \bar{\lambda} \sigma^{\mu \nu} F_{\mu \nu}\right] \tilde{G},
$$

where $F_{\mathrm{DSB}} \equiv\left[\sum_{i}\left\langle F_{i}\right\rangle^{2}\right]^{1 / 2}$ is the total $F$ vev generated by dynamical supersymmetry breaking. For simple cases where there is only one non-zero $\langle F\rangle, F_{\mathrm{DSB}}$ is equivalent to the $\left\langle F_{Z}\right\rangle$ appearing in Eq. (38), but in general, they need not be identical.

We will now describe several models, beginning with three supergravity theories. In supergravity, supersymmetry breaking is mediated through non-renormalizable gravitational interactions, and the large mass scale of Eq. (38) is the Planck mass $M_{\mathrm{Pl}}$. These interactions are generically present in all theories with supersymmetry and gravity, and supergravity theories are therefore natural models to consider. However, by their very nature, the coefficients of these non-renormalizable terms cannot be precisely determined without knowing the microscopic quantum theory of gravity from 
which they presumably derive. To proceed, various simple and phenomenologically attractive assumptions are made. These assumptions then fix the soft terms, allowing one to conduct concrete collider studies.

Another approach is to suppress the non-renormalizable supergravity terms and find new sources for soft terms. This requires new structure involving additional fields and interactions, but has the advantage that the soft terms are calculable in field theory. We describe models with gaugeand anomaly-mediated supersymmetry breaking, two prominent examples of this approach.

Finally, we conclude with comments on the implications of GUT and string models for soft supersymmetry-breaking parameters.

\subsection{Minimal Supergravity}

In supergravity, soft terms are generated by terms such as those in Eq. (38), where the large mass scale is $M=M_{\mathrm{Pl}}$. Soft scalar masses have the form $m_{i j}^{2}=\lambda_{i j} m_{0}^{2}$, where $m_{0} \equiv\langle F\rangle / M_{\mathrm{Pl}}$, and $i, j$ are generational indices. These terms generically mediate flavor-changing transitions that violate the constraints discussed in Sec. (3.3).

In minimal supergravity [31, 32, 33], one assumes that all of the soft scalar masses are given by a single parameter $m_{0}$ at some high scale, typically taken to be $M_{\mathrm{GUT}} \simeq 2 \times 10^{16} \mathrm{GeV}$. Similarly, one assumes a unified gaugino mass $M_{1 / 2}$, and a universal $A$-term parameter $A_{0}$. At $M_{\mathrm{GUT}}$, the model is completely specified by standard model parameters and $m_{0}, M_{1 / 2}$, $A_{0}, B$ and $\mu$.

The weak-scale MSSM Lagrangian is then determined by evolving all parameters to the weak-scale through renormalization group evolution. Renormalization group evolution of supersymmetry parameters is described by a complicated system of differential equations. Schematically, however, the 1-loop renormalization group equations are

$$
\begin{aligned}
\frac{d g}{d t} & \sim \frac{1}{16 \pi^{2}} g^{3} \\
\frac{d y}{d t} & \sim \frac{1}{16 \pi^{2}}\left[g^{2} y-y^{3}\right] \\
\frac{d M}{d t} & \sim \frac{1}{16 \pi^{2}} g^{2} M \\
\frac{d A}{d t} & \sim \frac{1}{16 \pi^{2}}\left[-g^{2} M-y^{2} A\right] \\
\frac{d m^{2}}{d t} & \sim \frac{1}{16 \pi^{2}}\left[g^{2} M^{2}-y^{2} A^{2}-y^{2} m^{2}\right],
\end{aligned}
$$


where $t \equiv \ln \left(Q_{0} / Q\right)$ with $Q_{0}$ some fixed renormalization scale, and positive numerical coefficients and gauge and flavor indices have been neglected. In evolving down from a high scale, gauge interactions raise $m^{2}$, while Yukawa interactions lower $m^{2}$. Unique among scalar mass parameters, $m_{H_{u}}^{2}$ is driven down by the large top quark Yukawa without a compensating positive contribution from the strong gauge interactions. It is typically driven negative, breaking electroweak symmetry. This feature of radiative electroweak symmetry breaking is often regarded as another success of supersymmetry.

At the weak scale, the supersymmetry parameters are constrained by the following tree-level relations:

$$
\begin{aligned}
\frac{1}{2} m_{Z}^{2} & =\frac{m_{H_{d}}^{2}-m_{H_{u}}^{2} \tan ^{2} \beta}{\tan ^{2} \beta-1}-|\mu|^{2} \\
2 B & =\left(m_{H_{u}}^{2}+m_{H_{d}}^{2}+2|\mu|^{2}\right) \sin 2 \beta .
\end{aligned}
$$

Since $m_{Z}$ is measured, these relations are used to exchange $B$ and $\mu$ at the high scale for $\tan \beta$ and $\operatorname{Arg}(\mu)$. In minimal supergravity, $\mu$ is assumed real, and so the fundamental parameters of minimal supergravity are

$$
m_{0}, M_{1 / 2}, A_{0}, \tan \beta, \operatorname{sign}(\mu) .
$$

The scale of supersymmetry breaking is set by the requirement that the soft terms are of the order of the weak scale, so $\langle F\rangle \sim m_{W} M_{\mathrm{Pl}} \sim 10^{20} \mathrm{GeV}^{2}$. By Eq. (39), the gravitino mass is also of the order of the weak scale. It is typically assumed to be heavier than the standard model superpartners. In this case, the gravitino is irrelevant for colliders, as its interactions are gravitational and so extremely weak.

The masses of the rest of the superpartners are determined by renormalization group evolution. At the weak scale, the gaugino mass parameters are given by

$$
\frac{M_{i}}{\alpha_{i}}=\frac{M_{1 / 2}}{\alpha_{\text {unif }}}
$$

in accord with Eq. (30). Numerically, this implies

$$
M_{1} \approx 0.4 M_{1 / 2}
$$

For the scalar masses, neglecting the effects of Yukawa couplings, we find 
the weak-scale values

$$
\begin{aligned}
& m_{Q}^{2}=m_{0}^{2}+6.3 M_{1 / 2}^{2} \\
& m_{U}^{2}=m_{0}^{2}+5.8 M_{1 / 2}^{2} \\
& m_{D}^{2}=m_{0}^{2}+5.8 M_{1 / 2}^{2} \\
& m_{L}^{2}=m_{0}^{2}+0.5 M_{1 / 2}^{2} \\
& m_{E}^{2}=m_{0}^{2}+0.15 M_{1 / 2}^{2} .
\end{aligned}
$$

If $m_{0}$ and $M_{1 / 2}$ are of the same order, the squark masses are dominated by $M_{1 / 2}$, while the slepton masses are dominated by $m_{0}$. Generically the renormalization group equations predict heavy squarks and light sleptons. The lightest scalars are $\tilde{\tau}_{R}$, followed by $\tilde{\mu}_{R}, \tilde{e}_{R}$, and the left-handed sleptons.

Minimal supergravity has a number of virtues. Grand unified boundary conditions are automatically incorporated. In addition, as is evident from Eqs. (50) and (51), in much of parameter space, the LSP is the lightest neutralino, and so is a possible dark matter candidate. The nature of the LSP in various regions of parameter space is presented in Fig. 8. For most of the region with $m_{0} \lesssim 1 \mathrm{TeV}$, the LSP is a Bino-like neutralino. Minimal supergravity also solves the supersymmetric flavor and CP problems, but only through ad hoc assumptions. From a universal scalar mass at $M_{\mathrm{GUT}}$, renormalization group evolution generates mass splittings and flavor mixing, but these are generally small. The assumption of a real $\mu$ parameter also guarantees the absence of supersymmetric CP violation, suppressing dangerous contributions to electric dipole moments.

To summarize, a typical minimal supergravity scenario predicts

$$
\begin{gathered}
\text { NLSP candidates : } \tilde{l}_{R} \text { or } \tilde{\chi}_{2}^{0}, \tilde{\chi}_{1}^{ \pm} \approx \tilde{W}^{0}, \tilde{W}^{ \pm} \\
\operatorname{LSP}: \tilde{\chi}_{1}^{0} \approx \tilde{B},
\end{gathered}
$$

where NLSP denotes the next-to-lightest supersymmetric particle, which is the lightest detectable in colliders. One therefore generally expects neutralinos, charginos, and sleptons to be produced in the greatest numbers at linear colliders.

\subsection{Focus Point Supersymmetry}

Perhaps the most straightforward solution to the flavor and CP constraints of Secs. (3.3) and (3.4) is to assume heavy superpartners. However, large supersymmetry parameters typically re-introduce the gauge hierarchy and so are considered unnatural. As an example, consider Eq. (46): if $\left|m_{H_{u}}^{2}\right| \gg$ 


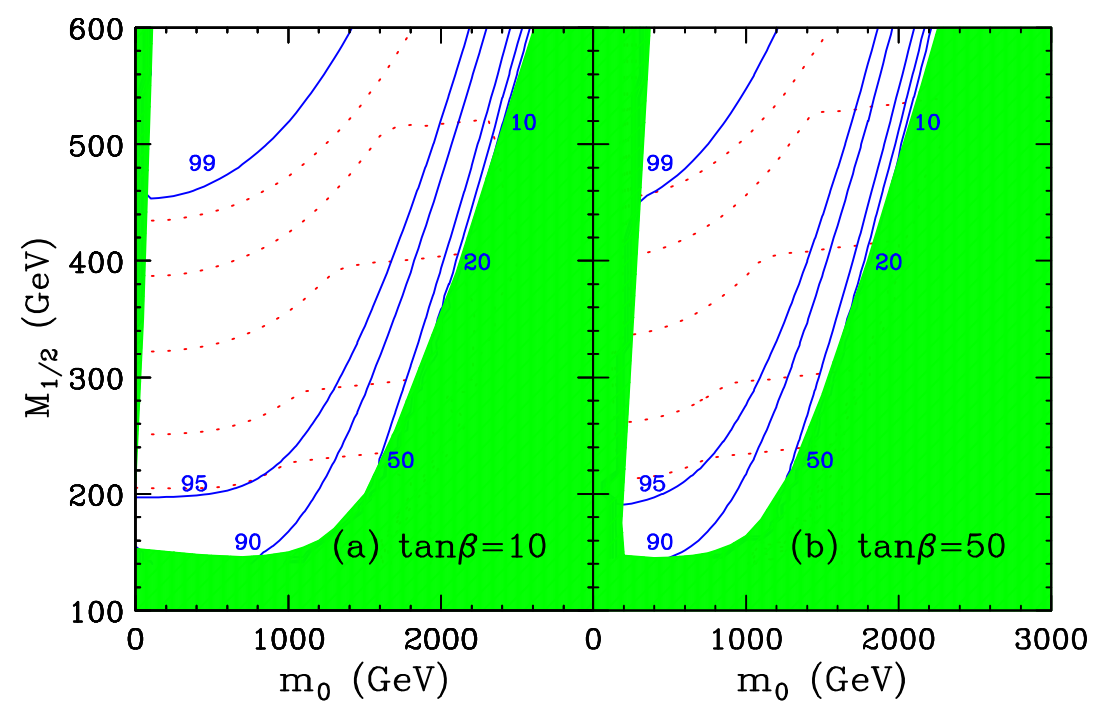

Fig. 8. The character of the LSP in minimal supergravity with $A_{0}=0$ and $\mu<0$ 34. In the unshaded regions, the LSP is the lightest neutralino, and contours of the gauginoness of the LSP $R_{\chi} \equiv\left|N_{11}\right|^{2}+\left|N_{12}\right|^{2}$ are given (in percent). In the narrow upper left shaded regions, the LSP is the lighter stau. The lower right shaded regions are excluded by bounds on the chargino mass.

$m_{Z}^{2}$, the parameter $\mu$ must be highly fine-tuned to cancel most of $m_{H_{u}}^{2}$ to give the correct $Z$ mass.

Naturalness does not require all supersymmetry-breaking parameters to be small, however. In focus point models [35, 36], large fundamental supersymmetry parameters do not imply large cancellations in Eq. (46). A simple example is provided by the minimal supergravity model just discussed. Rewriting Eq. (46) in terms of the fundamental parameters, we find through numerical analysis

$$
\frac{1}{2} m_{Z}^{2}=-0.04 m_{0}^{2}+8.8 M_{1}^{2}-|\mu|^{2} .
$$

The small $m_{0}^{2}$ coefficient implies that even large values of $m_{0}$ do not result in large fine-tuning in the electroweak potential. The small coefficient may be understood in terms of a focus point in the $m_{H_{u}}^{2}$ renormalization group trajectories, shown in Fig. 9. For a large range of initial values $m_{0}$, these trajectories focus to a point with $m_{H_{u}}^{2} \approx 0$ at the weak scale. Naturalness bounds $m_{H_{u}}^{2}$ at the weak scale, but constrains the GUT-scale parameter $m_{0}$ only loosely. While $m_{H_{u}}^{2}$ is insensitive to $m_{0}$, the masses of all sleptons and squarks are of order $m_{0}$, and so very heavy scalars are consistent with 


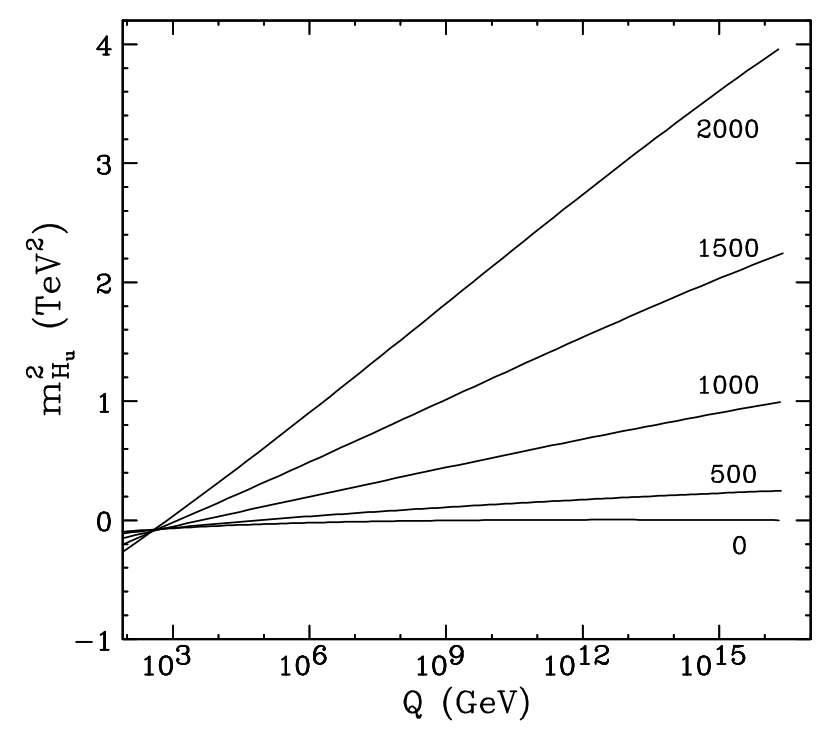

Fig. 9. Renormalization group trajectories of $m_{H_{u}}^{2}$ in minimal supergravity for various $m_{0}$ as labeled and $M_{1 / 2}=300 \mathrm{GeV}, A_{0}=0, \tan \beta=10$ [36]. These focus to a point at $Q \sim m_{W}$.

naturalness in minimal supergravity, while at the same time alleviating flavor and $\mathrm{CP}$ constraints 37.

The focus point region in minimal supergravity is the region of $m_{0} \gtrsim 1 \mathrm{TeV}$ in Fig. 8. From Eq. (53), we see that, for fixed $M_{1 / 2}$, large $m_{0}$ requires small $|\mu|$. This is also evident in Fig. 8, where, for large $m_{0}$ there is a region with $M_{1 / 2}$ and $|\mu|$ comparable, and therefore significant Higgsino content in the lightest neutralino. The prediction of focus point supersymmetry, then, is that, while sleptons and squarks are typically heavy, possibly even with multi- $\mathrm{TeV}$ masses beyond the LHC, the electroweak gauginos and Higgsinos are all light.

In fact, constraints on the dark matter relic density typically require significant gaugino-Higgsino mixing in focus point supersymmetry. The requirement that dark matter not overclose the universe implies that the processes of Fig. 3 be sufficiently efficient. In focus point supersymmetry, the processes mediated by sleptons and squarks are highly suppressed, and so dark matter annihilation relies on the processes with gauge boson final states. These processes are, however, absent for Bino-like LSPs, as they rely on gaugino-Higgsino mixing. As a result, dark matter constraints require gaugino-Higgsino mixing, and $|\mu|$ not too far above the gaugino mass 
parameters.

In focus point supersymmetry, then, all 6 charginos and neutralinos may be within reach of the linear collider, allowing detailed studies of these systems. A typical focus point spectrum is

$$
\begin{aligned}
\text { Heavy }: & \tilde{l}, \tilde{q} \\
\text { NLSP candidates : } & \tilde{\chi}_{2}^{0}, \tilde{\chi}_{1}^{ \pm} \approx \text { gaugino }- \text { Higgsino mixtures } \\
\text { LSP }: & \tilde{\chi}_{1}^{0} \approx \tilde{B}, \tilde{H}^{0} \text { mixture } .
\end{aligned}
$$

\subsection{Superheavy Supersymmetry}

Superheavy supersymmetry scenarios also alleviate the flavor and CP problems by decoupling. The soft Higgs masses $m_{H_{u}}^{2}$ and $m_{H_{d}}^{2}$ are constrained to the weak scale by naturalness. They are determined by the scalar mass renormalization group equation of Eq. (45). Note, however, that the other scalar mass parameters enter this renormalization group equation proportional to their Yukawa couplings.

This then naturally suggests a split spectrum with light third generation sfermions, and superheavy sfermions of the first two generations 38]. The light third generation sfermions preserve naturalness and do not induce violations of existing bounds, since low energy bounds on third generation observables are relatively weak. At the same time, superheavy sleptons and squarks of the first two generations, with masses of the order of $10 \mathrm{TeV}$, alleviate the most stringent flavor- and $\mathrm{CP}$-violating constraints, such as those in from the kaon system, and $\mu-e$ transitions, but do not sacrifice naturalness, since their effect on the $m_{H_{u}}^{2}$ and $m_{H_{d}}^{2}$ renormalization group equations are suppressed by tiny Yukawa couplings. The split spectrum of superheavy supersymmetry may be generated immediately upon supersymmetrybreaking [39] or by renormalization group evolution effects 40].

A typical superheavy supersymmetry spectrum is

$$
\begin{aligned}
\text { Superheavy : } & \tilde{e}, \tilde{\mu}, \tilde{u}, \tilde{d}, \tilde{c}, \tilde{s} \\
\text { NLSP candidates : } & \tilde{\tau}, \tilde{\nu}_{\tau}, \tilde{b}, \tilde{t}, \tilde{\chi}_{2}^{0}, \tilde{\chi}_{1}^{ \pm} \\
\text {LSP : } & \tilde{\chi}_{1}^{0} .
\end{aligned}
$$

\subsection{Gauge Mediation}

In supergravity scenarios, the soft terms are assumed to arise from nonrenormalizable gravitational interactions. As noted above, however, these 


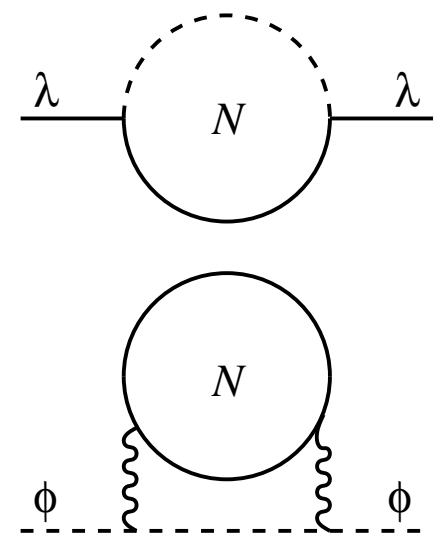

Fig. 10. Contributions to scalar and gaugino masses in gauge-mediated supersymmetry breaking.

are not calculable and ad hoc assumptions must be made to avoid constraints from flavor and $\mathrm{CP}$ bounds. In gauge-mediated supersymmetry breaking, one assumes that $\langle F\rangle \ll 10^{20} \mathrm{GeV}^{2}$, so that these troublesome gravitational contributions to soft masses are highly suppressed.

Of course, weak-scale soft terms must still be generated. This is achieved by introducing $N$ "messenger fields" with mass $M_{\text {mess }}$ 41, 42]. These have standard model gauge quantum numbers and generate MSSM soft masses at the scale $M_{\text {mess }}$ of the form

$$
\begin{aligned}
& M_{i}=N \frac{\left.g_{i}^{2}\right|_{Q=M_{\text {mess }}}}{16 \pi^{2}} \frac{\langle F\rangle}{M_{\text {mess }}} \\
& m_{\tilde{f}}^{2}=2 N \sum_{i} C_{i f}\left[\frac{\left.g_{i}^{2}\right|_{Q=M_{\text {mess }}}}{16 \pi^{2}}\right]^{2}\left[\frac{\langle F\rangle}{M_{\text {mess }}}\right]^{2}
\end{aligned}
$$

through diagrams such as those of Fig. 10. Here $C_{\text {if }}$ are coefficients determined by the sfermions' gauge representations. Yukawa effects are typically small and may be neglected. These soft masses are therefore dependent only on standard model gauge quantum numbers to an excellent approximation, and gauge mediation therefore elegantly predicts flavor-blind soft terms, which suppress contributions to flavor violation. Generically, CP-violating EDMs are still problematic, however.

The gaugino and sfermion masses of Eq. (57) are then evolved from the messenger scale to the weak scale. The gaugino masses are in the same proportion as in minimal supergravity, even though they are never unified, 
but the scalar masses are very different. The scalar spectrum is determined by only three parameters in the simplest models: $\langle F\rangle, M_{\text {mess }}$, and $N$. The possibility of distinguishing such spectra from supergravity predictions will be discussed in Sec. (9).

For colliders, a key feature of gauge-mediated supersymmetry breaking is the prediction of $m_{\tilde{G}} \sim\left\langle F_{\mathrm{DSB}}\right\rangle / M_{\mathrm{Pl}} \ll m_{\text {Weak }}$. The gravitino is therefore always the LSP. The NLSP is determined by a number of factors. For example, although the gaugino masses grow linearly with $N$, the scalar masses grow only as $\sqrt{N}$. For low $N$, the NLSP is typically a neutralino, but for larger $N$, the NLSP is typically a slepton [43. This may be seen in Fig. 11, where the character of the NLSP in various regions of parameter space is shown. In general, then, we have the possibilities

$$
\begin{gathered}
\text { NLSP candidates : } \tilde{\chi}_{1}^{0}, \tilde{l} \\
\text { LSP : } \tilde{G} .
\end{gathered}
$$

The NLSP decays to the gravitino with decay length

$$
L \sim 0.1 \mathrm{~mm} \times\left[\frac{E_{\mathrm{NLSP}}^{2}-m_{\mathrm{NLSP}}^{2}}{m_{\mathrm{NLSP}}^{2}}\right]^{\frac{1}{2}}\left[\frac{\sqrt{F_{\mathrm{DSB}}}}{10^{5} \mathrm{GeV}}\right]^{4}\left[\frac{100 \mathrm{GeV}}{m_{\mathrm{NLSP}}}\right]^{5} .
$$

$\sqrt{F_{\mathrm{DSB}}}$ may range from $10^{5} \mathrm{GeV}$ to $10^{9} \mathrm{GeV}$, where the lower limit is set by lower bounds on superpartner and messenger masses and the upper limit follows from requiring the supergravity contributions to be sub-dominant. For low values of $\sqrt{F_{\mathrm{DSB}}}$ in this range, the NLSP decays within collider detectors, producing signals with energetic photons and leptons, depending on what sparticle is the LSP [45]. However, for large $\sqrt{F_{\mathrm{DSB}}}$, the NLSP may travel macroscopic distances before decaying. In the case of neutralino NLSPs, this produces the conventional missing energy signals of supersymmetry. For slepton NLSPs, however, non-relativistic sleptons may produce highly-ionizing charged tracks and tracks with unusual time-of-flight signatures.

\subsection{Anomaly Mediation}

In anomaly-mediated supersymmetry breaking [46, 47], the dangerous supergravity contributions are suppressed geometrically - supersymmetrybreaking fields are placed on one 3-brane, with MSSM fields on another, as shown in Fig. 12. The usual supergravity contributions are then suppressed because the supersymmetry-breaking and MSSM wave functions are separated in space. 


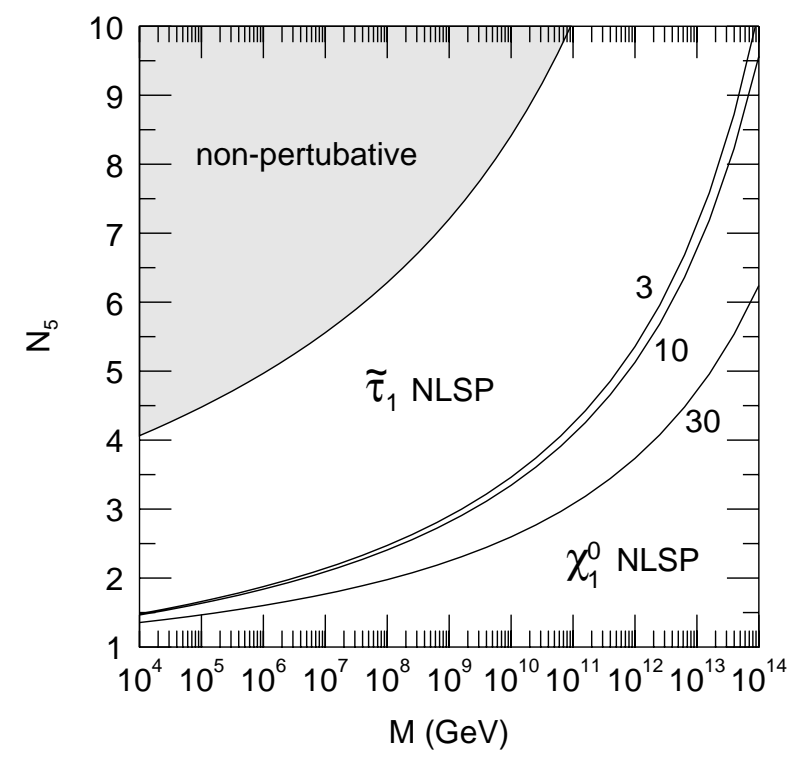

Fig. 11. The NLSP in various regions of minimal gauge-mediation model parameter space [44]. $M$ is the messenger mass or scale, and $N_{5}$ is the number of messenger multiplets. In the region above the labeled contours, the NLSP is a stau; in the region below, the NLSP is a neutralino. The boundary between the two regions depends slightly on $\tan \beta$; the boundaries for $\tan \beta=3,10$, and 30 are shown. In the shaded region, gauge coupling constants become non-perturbative below the GUT scale under two-loop renormalization group evolution.

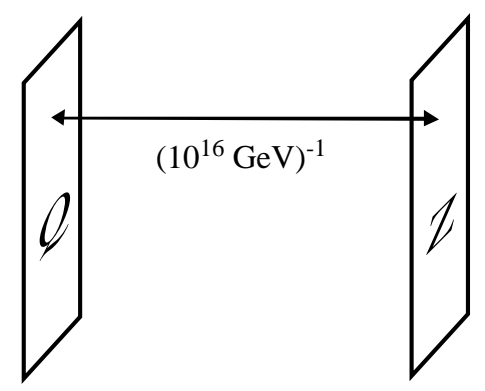

Fig. 12. Fields in anomaly-mediation: MSSM fields $Q$ are separated from supersymmetry-breaking fields $Z$ in extra dimensions.

In contrast to gauge mediation, however, in anomaly mediation, the soft terms are generated by loop-suppressed supergravity contributions of order $\sim\left(1 / 16 \pi^{2}\right)\langle F\rangle / M_{\mathrm{Pl}}$. These arise from the Weyl rescaling anomaly. They are 
always present, but are typically dominated by tree-level contributions in conventional four-dimensional supergravity. The required supersymmetrybreaking scale is therefore larger than in conventional supergravity, and the gravitino mass is of order $100 \mathrm{TeV}$. As with gauge mediation, the soft terms generated are essentially determined by gauge couplings, and so solve the supersymmetry flavor problem through degeneracy. The minimal models predict tachyonic sleptons - this catastrophe may be avoided either by assuming an additional contribution to scalar masses 48 or by postulating other additional structure [49]. Also, as in gauge-mediated models, additional structure is typically required to naturally satisfy EDM constraints.

The most striking phenomenological feature for colliders is the gaugino mass spectrum. The gaugino masses generated by the Weyl anomaly are $M_{i} \propto\left|b_{i} g_{i}^{2}\right|$, where $b_{i}$ is the one-loop $\beta$-function coefficient for the corresponding gauge group in the MSSM. In the MSSM, $b_{i}=(-33 / 5,-1,3)$, and the Wino mass is therefore predicted to be the smallest. The lightest states are then a triplet of Winos, and the typical spectrum is

$$
\begin{aligned}
\mathrm{NLSP}: & \tilde{\chi}_{1}^{ \pm} \approx \tilde{W}^{ \pm} \\
\mathrm{LSP}: & \tilde{\chi}_{1}^{0} \approx \tilde{W}^{0} .
\end{aligned}
$$

These Wino states are extraordinarily degenerate - at tree-level, their splitting is typically of order $1 \mathrm{MeV}$. One-loop contributions raise this to $150 \mathrm{MeV}$ or more, allowing $\tilde{\chi}_{1}^{ \pm} \rightarrow \tilde{\chi}_{1}^{0} \pi^{ \pm}$[50. However, the decay length may be of order $1 \mathrm{~cm}$, and the resulting pion is very soft, leading to a peculiar and qualitatively new supersymmetry signature at colliders.

\subsection{GUT and Planck Scale Frameworks}

In the discussion above, we noted that the soft terms are in principle not predictable in supergravity. Properties such as scalar universality appeared as phenomenological assumptions, motivated by simplicity and experimental constraints. This is not the complete story, however. As also noted above, one strong motivation for supersymmetry is the unification of forces, including the three standard model forces and also gravity. The circle of ideas surrounding GUTs and string theory therefore naturally have implications for the GUT scale boundary conditions.

As an example, in string theory, the low energy effective theory is expected to be supersymmetric. In addition to the MSSM fields, this theory also contains many light scalar fields, including the dilaton and moduli, 
with gravitationally-suppressed couplings. The dilaton superfield's scalar component is the source of gauge and gravitational couplings, and its $F$ term may break supersymmetry if it condenses. If this is the main source of supersymmetry breaking, the resulting models, called dilaton-dominated supersymmetry breaking models [51], predict mass spectra similar to minimal supergravity, but with specific relations among the average scalar mass, $M_{1 / 2}$, and $A_{0}$ at the Planck scale. Small splittings of scalar masses are introduced by moduli fields, and there are sum rules for scalar masses.

At slightly lower scales, consider GUTs motivated by the unification of couplings described in Sec. (3.1). In these theories, the MSSM gauge multiplets are merged into GUT multiplets, and have GUT interactions. For example, in $\mathrm{SO}(10)$ GUTs, the $\tau$ and $t$ superfields are unified in one GUT representation. As a result, above the GUT scale, renormalization group evolution of the $\tilde{\tau}$ mass includes radiative corrections arising from the triplet Higgs boson with strength proportional to the top Yukawa coupling [52]. This effect is not present for selectrons and smuons, leading to a substantial splitting of slepton masses, even if they are initially degenerate. GUT symmetries may also lead to alternative parameterizations of the GUT scale boundary conditions. For example, in $\mathrm{SO}(10)$ theories, the Higgs and matter multiplets are members of separate multiplets, the $\mathbf{1 0}$ and $\mathbf{1 6}$ representations, respectively. Gauge symmetry does not require their scalar masses to be unified. Furthermore, even if unified, $\mathrm{SO}(10)$ breaking to $\mathrm{SU}(5)$ as an intermediate step will split these scalar masses by $D$-terms 53,54 . As a result, the Higgs and matter scalar masses are more generally parameterized by two independent parameters at the GUT scale, opening up many new possibilities in the low energy MSSM spectrum.

While we cannot describe all models investigated to date here, we can extract important lesson from the above discussion. The soft terms of the MSSM may carry the imprint of interactions that are not amenable to direct experimental investigation. Measurements of soft mass relations may therefore shed light on physics at the highest energy scales.

\section{Slepton Studies}

We now turn to specific studies of supersymmetry at the linear collider. We begin with sleptons, which are the lightest observable superpartners in many models. As we will see, slepton studies may shed light on a large number of important questions. They also provide an arena for exploiting many of the most powerful techniques available to study new physics the 
linear collider.

\subsection{Signal and Background}

Slepton pair production proceeds through $s$-channel gauge bosons, $e^{+} e^{-} \rightarrow$ $\gamma, Z \rightarrow \tilde{l}^{+} \tilde{l}^{-}$and $e^{+} e^{-} \rightarrow Z \rightarrow \tilde{\nu} \tilde{\nu}^{*}$. In the case of 1 st generation sleptons, there are also contributions from $t$-channel neutralino and chargino exchange. In the presence of supersymmetric LFV, $t$-channel contributions are also present for the 2nd and 3rd generations. We will assume lepton flavor is conserved here, deferring discussion of LFV to Sec. (5.4).

Let us focus on supergravity scenarios with $R$-parity conservation. When sleptons are heavier than neutralinos or charginos, they decay through

$$
\begin{aligned}
& \tilde{l} \rightarrow \tilde{\chi}_{i}^{0} l, \tilde{\chi}_{i}^{-} \nu_{l} \\
& \tilde{\nu} \rightarrow \tilde{\chi}_{i}^{+} l, \tilde{\chi}_{i}^{0} \nu_{l} .
\end{aligned}
$$

Sleptons have spin 0 and so decay isotropically in their rest frames. Righthanded sleptons decay dominantly to the lighter neutralino with the largest $\tilde{B}$ component. Left-handed sleptons decay primarily to the lighter neutralinos and charginos with the largest $\tilde{W}$ components. When decay into heavy charginos or neutralinos is possible, sleptons may initiate cascade decays ending in the LSP with many visible particles produced along the way.

We now focus on the simplest case in which a charged slepton is the NLSP, and the LSP is the lightest neutralino $\tilde{\chi}_{1}^{0}$. The signal is then

$$
e^{+} e^{-} \rightarrow \tilde{l}^{+} \tilde{l}^{-} \rightarrow l^{+} l^{-} \tilde{\chi}_{1}^{0} \tilde{\chi}_{1}^{0} .
$$

The momenta of the two leptons may be measured precisely, but the two LSPs escape detection. The signal is same flavor, opposite sign leptons with missing transverse momentum $\not_{T}$.

The major standard model background to this process is $W$ pair production, leading to $e^{+} e^{-} \rightarrow W^{+} W^{-} \rightarrow l^{+} \nu_{l} l^{\prime-} \bar{\nu}_{l^{\prime}}$. The total $W^{+} W^{-}$cross section is enormous compared to typical supersymmetry cross sections, but it proceeds largely through $t$-channel exchange of the light electron, and so is very forward-peaked. The total $W^{+} W^{-}$cross section may therefore be reduced from about $10 \mathrm{pb}$ at $\sqrt{s}=500 \mathrm{GeV}$ to less than $2 \mathrm{pb}$ with the requirement $|\cos \theta|<0.7$, where $\theta$ is the polar angle of the $W$ relative to

${ }^{\mathrm{b}}$ In general, $R$-parity violating theories are not difficult to study at linear colliders, as the relevant backgrounds are not overwhelming and all superpartner decay products are visible, allowing full momentum and energy reconstruction [55]. 
the beam axis. Of this, only $1 \%$ produces a given same flavor final state. For comparison, the typical $\tilde{\mu}_{R}^{+} \tilde{\mu}_{R}^{-}$production cross section is $50 \mathrm{fb}^{-1}$ not far above threshold, and such processes are well-represented in the central region.

Beam polarization may also greatly reduce the $W^{+} W^{-}$background 56 , 57]. An electron's spin along its direction of motion is essentially determined by its chirality. We define polarizations for the electron and positron beams through

$$
\begin{aligned}
& P_{e^{-}}=\frac{N\left(h=\frac{1}{2}\right)-N\left(h=-\frac{1}{2}\right)}{N\left(h=\frac{1}{2}\right)+N\left(h=-\frac{1}{2}\right)} \\
& P_{e^{+}}=\frac{N\left(\bar{h}=-\frac{1}{2}\right)-N\left(\bar{h}=\frac{1}{2}\right)}{N\left(\bar{h}=\frac{1}{2}\right)+N\left(\bar{h}=-\frac{1}{2}\right)},
\end{aligned}
$$

where $h$ and $\bar{h}$ are the $e^{-}$and $e^{+}$helicities, respectively. The cross section for pair production of any particles may then be expressed as

$$
\begin{aligned}
& \frac{d}{d \cos \theta} \sigma(h, \bar{h})=\frac{1}{32 \pi s} \beta_{f} \sum_{h, \bar{h}}|\mathcal{M}(h, \bar{h})|^{2} \\
& \times \frac{1}{4}\left[1+(-)^{-1 / 2+h} P_{e^{-}}\right]\left[1+(-)^{1 / 2+\bar{h}} P_{e^{+}}\right] .
\end{aligned}
$$

Here $\beta_{f}=\left(1-m_{f}^{2} / E_{\text {beam }}^{2}\right)^{1 / 2}$ is the velocity of the final state particles, where $m_{f}$ is their mass, and $E_{\text {beam }}=\sqrt{s} / 2$ is the beam energy. $\mathcal{M}(h, \bar{h})$ is the amplitude for incoming electrons and positrons with helicities $h$ and $\bar{h}$, respectively, and depends on $\cos \theta$.

Much of $W$ pair production proceeds through $\bar{\nu} \gamma_{\mu} P_{L} e W^{\mu}$ couplings. For right-polarized beams, then, the production cross section is reduced by the factor $\left(1-P_{e^{-}}\right)\left(1-P_{e^{+}}\right)$relative to the unpolarized case. For $P_{e^{-}}=0.8$ and unpolarized positrons, the cross section is reduced by a factor of 5 . In the limit where beam polarization is perfect $\left(P_{e^{-}}=P_{e^{+}}=1\right)$ and $\sqrt{s} \gg m_{W}$, the production cross section of transverse $W$ bosons goes to zero and the cross section is dominated by the process with longitudinal $W$ bosons in the $s$-channel.

Another important background is $e^{+} e^{-} \rightarrow e^{+} e^{-} l^{+} l^{-}$. This is a background to slepton pair production when the two photons radiated off from the initial-state $e^{+}$and $e^{-}$collide to produce a $l^{+} l^{-}$pair, while the initialstate $e^{+}$and $e^{-}$escape into the beam pipe. Note that beam polarization

${ }^{\mathrm{c}}$ In this limit, the cross section reduces to that of Goldstone boson production, as required by the equivalence theorem. 
cannot reduce this background. In current linear collider detector designs, electrons and positrons traveling within $\theta_{\text {min }} \sim 5 \mathrm{mrad}$ are undetected, as this region is not covered by luminosity monitors. The maximum $p_{T}$ carried by these electrons and positrons is then

$$
\not p_{T}^{\max }=2.5 \mathrm{GeV} \frac{\theta_{\min }}{5 \mathrm{mrad}} \frac{E_{\text {beam }}}{500 \mathrm{GeV}} .
$$

Supersymmetric events with $\not p_{T} \lesssim 2.5 \mathrm{GeV}$ are therefore obscured by this background. Of course, the two-photon background may be greatly reduced with $\not_{T}$ and acoplanarity cuts, with little impact on most supersymmetry signals.

\subsection{Slepton Masses}

\section{Kinematic Endpoints}

At linear colliders, the parton energy is fixed, neglecting the effects of initial state radiation, beam energy spread, and beamstrahlung. To a reasonable approximation, then, sleptons are produced with energy equal to the beam energy $E_{\text {beam }}=\sqrt{s} / 2$, and velocity

$$
\beta_{\tilde{l}}=\frac{p}{E_{\text {beam }}}=\sqrt{1-\frac{m_{\tilde{\imath}}^{2}}{E_{\text {beam }}^{2}}} .
$$

For sleptons produced not too far above threshold, as will typically be the case at least initially, $\beta_{\tilde{l}}$ is substantially smaller than 1 .

The slepton then decays to $l+\tilde{\chi}_{1}^{0}$. In the slepton's rest frame, the lepton's energy is

$$
E_{l}^{*}=\frac{m_{\tilde{l}}^{2}-m_{\chi}^{2}+m_{l}^{2}}{2 m_{\tilde{l}}},
$$

where the lepton's mass may be neglected. Boosting to the lab frame, the lepton's energy is

$$
E_{l}(\cos \theta)=E_{l}^{*} \frac{1+\beta_{\tilde{l}} \cos \theta}{\sqrt{1-\beta_{\tilde{l}}^{2}}},
$$

where $\theta$ is the polar angle of $l$ in the slepton rest frame measured relative to the slepton's boost direction. In the lab frame, the lepton's energy distribution is therefore flat.

From Eq. (69), one can see that, provided $\beta_{\tilde{l}}$ is significantly less than 1 , the $\tilde{l}$ and $\tilde{\chi}_{1}^{0}$ masses may be well-determined by measuring the two endpoints 


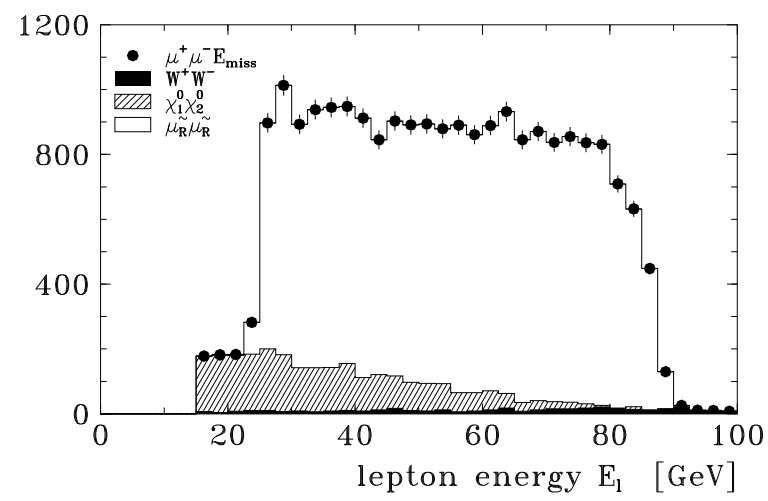

Fig. 13. Muon energy distribution from the signal $e_{R}^{-} e_{L}^{+} \rightarrow \tilde{\mu}_{R}^{+} \tilde{\mu}_{R}^{-} \rightarrow \mu^{+} \tilde{\chi}_{1}^{0} \mu^{-} \tilde{\chi}_{1}^{0}$ (open), and the dominant backgrounds (shaded), for $\sqrt{s}=320 \mathrm{GeV}$ and an integrated luminosity of $160 \mathrm{fb}^{-1}\left[58\right.$. The underlying supersymmetric masses are $m_{\tilde{\mu}_{R}}=132 \mathrm{GeV}$ and $m_{\tilde{\chi}_{1}^{0}}=71.9 \mathrm{GeV}$.

of the lepton energy distribution 56, 57. The relations between the masses and endpoints are

$$
\begin{aligned}
m_{\tilde{l}}^{2} & =\frac{s E_{\max } E_{\min }}{\left(E_{\max }+E_{\min }\right)^{2}} \\
1-\frac{m_{\tilde{\chi}_{1}^{0}}^{2}}{m_{\tilde{l}}^{2}} & =2 \frac{E_{\max }+E_{\min }}{\sqrt{s}} .
\end{aligned}
$$

In Fig. 13 we show the muon energy distribution from $\tilde{\mu}_{R}$ pair production, along with the dominant backgrounds [58]. The distribution is not perfectly flat: this distortion is caused by initial state radiation, beam energy spread, and beamstrahlung, along with acoplanarity cuts. Such effects smear the energy distribution near the edges and must be corrected through measurements of beam properties and Monte-Carlo simulations.

Doing so, one finds that $m_{\tilde{\mu}_{R}}$ can be measured to within $0.4 \mathrm{GeV}$ from kinematic endpoints, assuming an integrated luminosity of $160 \mathrm{fb}^{-1}$ for this case. Of course, the same measurement can be done for selectrons, and the precision of the mass measurement is of the same order [59]. This provides an immediate and stringent test of scalar mass relations, such as 


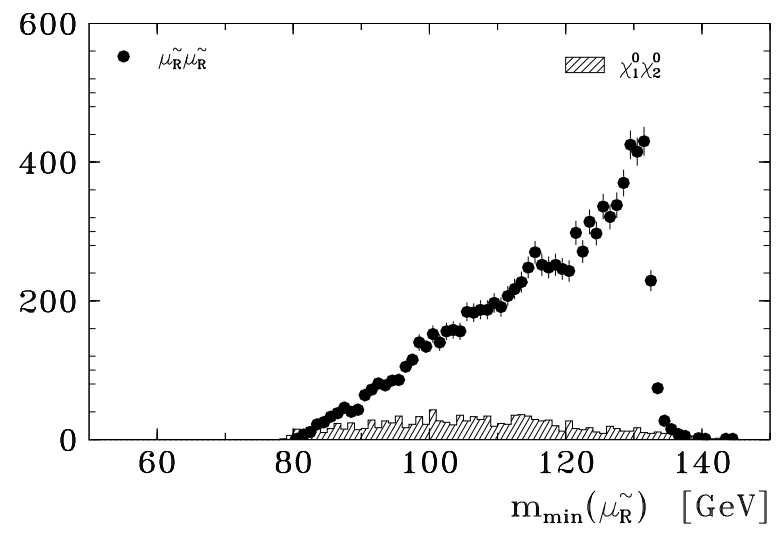

Fig. 14. Minimum mass distribution for smuons from the process $e_{R}^{-} e_{L}^{+} \rightarrow \tilde{\mu}_{R}^{+} \tilde{\mu}_{R}^{-} \rightarrow$ $\mu^{+} \tilde{\chi}_{1}^{0} \mu^{-} \tilde{\chi}_{1}^{0}$, for $\sqrt{s}=320 \mathrm{GeV}$ and an integrated luminosity of $160 \mathrm{fb}^{-1}$ [10]. The underlying supersymmetry parameters are $m_{\tilde{\mu}_{R}}=132 \mathrm{GeV}$ and $m_{\tilde{\chi}_{1}^{0}}=71.9 \mathrm{GeV}$.

the universality of scalar masses at the GUT scale discussed in Sec. (4.1).

\section{Minimum Mass Method}

In the kinematic endpoint method, the fact that leptons come in pairs in slepton events is never used. An analysis that incorporates this pairing information is the minimum mass method [12], where for each event, one determines the minimum slepton mass consistent with the measured lepton momenta and a postulated $\tilde{\chi}_{1}^{0}$ mass. The minimum mass distribution peaks sharply at the actual mass [12, 60, 61, 10], as may be seen in Fig. 14, making possible mass measurements that are more precise than those from the kinematic endpoint method discussed above. For scenarios in which the $\tilde{\chi}_{1}^{0}$ mass is well-known from some other process, such as chargino production, the minimum mass method provides another avenue for precise slepton mass determination.

\section{Threshold Scans}

Slepton masses may also be determined through threshold scans 62 , 58, 63, 64, 65, 66, 67. For pair-production of right-handed sleptons, the required initial state has angular momentum $J_{z}=1$. Since the final state particles have spin 0 , they must be produced in a $P$ wave, and the production cross section grows as $\beta^{3}$ near threshold, in contrast to fermion pair production, where the cross section grows as $\beta$. The measurement of this 
behavior is a telling check that sleptons are, in fact, scalars. An example of this threshold behavior is given in Fig. 15. In view of the $\beta^{3}$ suppression, such measurements require high luminosity, and finite width effects, threshold corrections, and sub-dominant diagrams must all be carefully controlled. However, $e^{+} e^{-}$threshold scans may allow precise mass measurements at the $100 \mathrm{MeV}$ level, assuming luminosities of $\sim 100 \mathrm{fb}^{-1}$ [58, 64].

In $e^{-} e^{-}$collisions, the relevant initial state for $\tilde{l}_{R}^{+} \tilde{l}_{R}^{-}$production has angular momentum $J_{z}=0$, and so slepton pair production has $\beta$ threshold behavior [62, 64]. Cross sections for $\tilde{e}_{R}$ pair production in $e^{-} e^{-}$and $e^{+} e^{-}$ modes are compared in Fig. 15. Mass measurements with $100 \mathrm{MeV}$ precision can be achieved with a total luminosity of $\mathcal{O}(1) \mathrm{fb}^{-1}$, two orders of magnitude less than required in $e^{+} e^{-}$collisions for similar precision 64. The full arsenal of linear collider modes may even allow one to extend this mass measurement to the rest of the first-generation sleptons through a series of $\beta$ threshold scans: $e^{-} e^{-} \rightarrow \tilde{e}_{R}^{-} \tilde{e}_{R}^{-}$yields $m_{\tilde{e}_{R}} ; e^{+} e^{-} \rightarrow \tilde{e}_{R}^{ \pm} \tilde{e}_{L}^{\mp}$ yields $m_{\tilde{e}_{L}} ; e^{+} e^{-} \rightarrow \tilde{\chi}_{1}^{+} \tilde{\chi}_{1}^{-}$yields $m_{\tilde{\chi}_{1}^{ \pm}} ;$and $e^{-} \gamma \rightarrow \tilde{\nu}_{e} \tilde{\chi}_{1}^{-}$yields $m_{\tilde{\nu}_{e}}$ [68]. If completed, this would yield a high precision test of Eq. (25), a robust prediction of supersymmetry and gauge invariance, and possibly also a high precision measurement of $\tan \beta$.

\section{Mass Measurements at the $\mathrm{LHC}$}

The LHC may also measure superpartner masses with some precision. At hadron colliders, the parton-parton center-of-mass energy is unknown. In supersymmetric events, typically two LSPs are missing, but only the total missing momentum can be reconstructed, and there is a large uncertainty in the longitudinal momentum of the system. Nevertheless, it is still possible to obtain reliable mass measurements from cascade decays if one can isolate an invariant mass distribution from a particular step in a cascade decay 69, 70]. For example, in the process $\tilde{\chi}_{2}^{0} \rightarrow \tilde{l}_{R} l \rightarrow \tilde{\chi}_{1}^{0} l^{+} l^{-}$, the $l^{+} l^{-}$invariant mass distribution is predicted to be proportional to $m_{l l}$, with maximum

$$
m_{l l}^{\max }=\sqrt{\frac{\left(m_{\tilde{\chi}_{2}^{0}}^{2}-m_{\tilde{l}_{R}}^{2}\right)\left(m_{\tilde{l}_{R}}^{2}-m_{\tilde{\chi}_{1}^{0}}^{2}\right)}{m_{\tilde{l}_{R}}^{2}}} .
$$

The endpoint of the $m_{l l}$ distribution is therefore determined by the three masses involved in the cascade decay. An example of this mass distribution is shown in Fig. 16. When the jets from the parent decay $\tilde{q} \rightarrow \tilde{\chi}_{2}^{0} q$ are also identified, the number of identified end points increases to four: the upper endpoints of the $m_{j l}$ and $m_{j l l}$ distributions, and the lower endpoint of the $m_{j l l}$ distribution for $m_{l l}>m_{l l}^{\max } / \sqrt{2}$. In principle, then, all 4 masses 

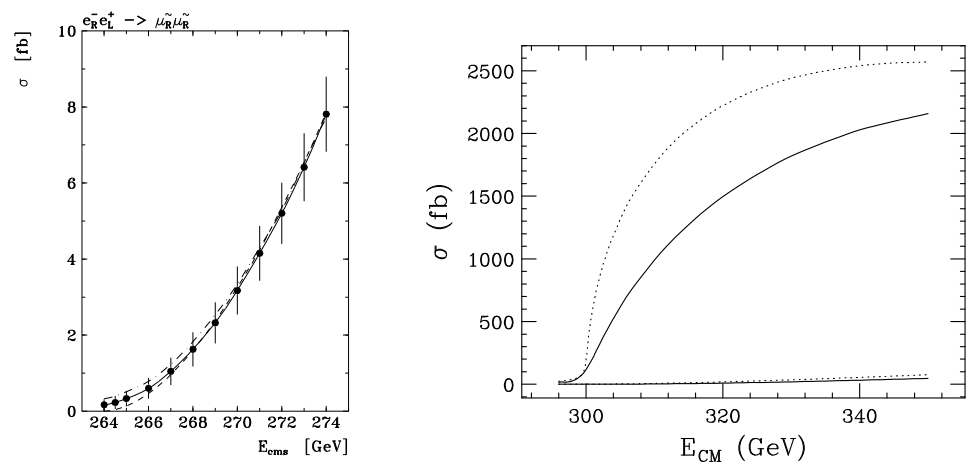

Fig. 15. Left: Threshold cross sections for $e^{+} e^{-} \rightarrow \tilde{\mu}_{R}^{+} \tilde{\mu}_{R}^{-}$, with $m_{\tilde{\mu}}=132 \mathrm{GeV}$ and smuon width $\Gamma_{\tilde{\mu}_{R}}=0.3$ (solid) and 0 and $0.6 \mathrm{GeV}$ (dashed) 63. The error bars are for a 10 point scan with total integrated luminosity $\mathcal{L}=100 \mathrm{fb}^{-1}$. Right: Threshold behavior for $\sigma\left(e^{-} e^{-} \rightarrow \tilde{e}_{R}^{-} \tilde{e}_{R}^{-}\right)$(upper two contours) and $\sigma\left(e^{+} e^{-} \rightarrow \tilde{e}_{R}^{+} \tilde{e}_{R}^{-}\right.$) (lower two contours) for $m_{\tilde{e}_{R}}=150 \mathrm{GeV}$ and $M_{1}=100 \mathrm{GeV}$ and beam polarizations $P_{e^{-}}=0.8$ and $P_{e^{+}}=0$ [64]. In each pair, the dotted curve neglects all beam effects, and the solid curve includes the initial state radiation, beamstrahlung, and beam energy spread of flat beams.

entering the cascade may be reconstructed. The resulting mass determinations for two sample points are also shown in Fig. 16. The mass difference $m_{\tilde{l}_{R}}-m_{\tilde{\chi}_{1}^{0}}$ is determined rather precisely. Note, however, that the absolute sparticle masses are poorly constrained, with uncertainties of the order of several tens of $\mathrm{GeV}$.

The example above illustrates the complementarity of the LHC and the linear collider. At the LHC, squarks and gluinos are produced with large cross sections, and their cascade decays typical include many charginos and neutralinos, as well as charged sleptons and sneutrinos. Distributions within these cascade decays may provide important kinematic information. The LHC therefore provides a "top view" of the superparticle spectrum. On the other hand, the linear collider produces the lighter sparticles first and determines their properties systematically from the bottom up. Remarkably, the uncertainties of masses and other parameters may be determined at each step with percent level uncertainty. Combining the data from the linear collider with that available at the LHC, all sparticle masses may be determined with a precision of the order of a few percent.

It is also noteworthy that, while the LHC determines decay patterns, 

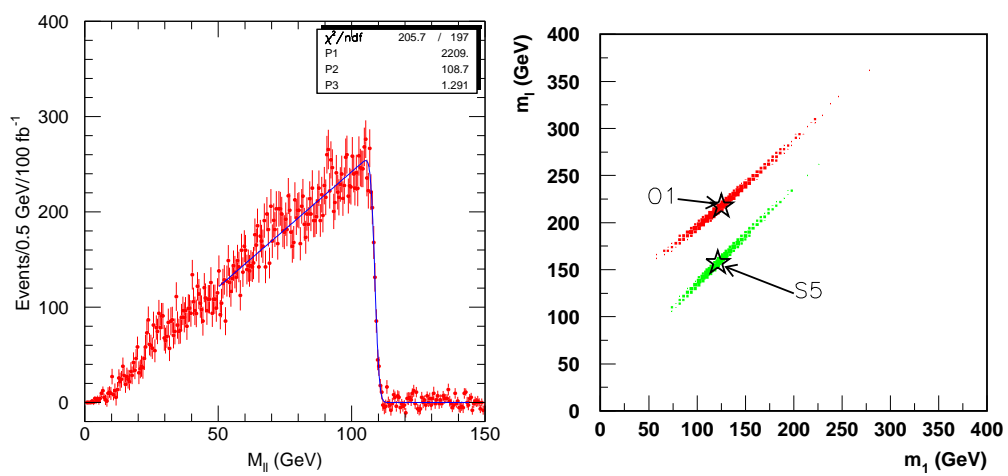

Fig. 16. Left: The $e^{+} e^{-}+\mu^{+} \mu^{-}-e^{ \pm} e^{\mp}$ mass distribution for LHC minimal supergravity Point 5 with $\tilde{\chi}_{2}^{0} \rightarrow \tilde{l} l \rightarrow \tilde{\chi}_{1}^{0} l^{+} l^{-}$71. Right: Scatter plot of reconstructed values of the $\tilde{l}_{R}$ and $\tilde{\chi}_{1}^{0}$ masses for LHC point 5 (S5) and for a different model (O1) using the decay chain $\tilde{q}_{L} \rightarrow \tilde{\chi}_{2}^{0} q \rightarrow \tilde{l}_{R} l q \rightarrow \tilde{\chi}_{1}^{0} l l q \mid 72$.

detailed determinations of the spins and couplings of sleptons and other particles is difficult. Using polarized beams, the linear collider may determine these slepton properties, as we now describe.

\subsection{Polarized Cross Sections}

We now consider selectron pair production. As noted in Eq. (65), cross sections for various beam polarizations are the sum of four independent polarized cross sections determined by the amplitudes $\mathcal{M}(h, \bar{h})$. Each $\mathcal{M}(h, \bar{h})$ is a function of gauge-sfermion-sfermion and gaugino-sfermion-fermion couplings. For $\tilde{e}_{R}^{+} \tilde{e}_{R}^{-}$production,

$$
\begin{aligned}
i \mathcal{M}(h, \bar{h})= & -i \lambda e^{i \lambda \phi} \sin \theta s \beta_{f}\left[\frac{g^{2}}{\cos ^{2} \theta_{W}} \frac{A_{h} A_{\frac{1}{2}}}{s-m_{Z}^{2}+i \Gamma_{Z}}\right. \\
& \left.+\frac{e^{2}}{s}+\frac{\left(1 \pm(-)^{\bar{h}+\frac{1}{2}}\right)}{2} \sum_{j} \frac{1}{2} \frac{\left|A_{j R}^{2}\right|}{t-m_{\tilde{\chi}_{j}}^{2}}\right],
\end{aligned}
$$

where $h= \pm 1 / 2$ is the helicity of the initial state electron, $\bar{h}= \pm 1 / 2$ is the helicity of the initial state positron, and $\lambda \equiv h-\bar{h}$. The angles $\theta$ and $\phi$ specify the $\tilde{e}_{R}^{-}$production direction in polar coordinates, $\beta_{f}$ is the selectron velocity, and $t=-\frac{s}{4}\left(1-2 \cos \theta \beta_{f}+\beta_{f}^{2}\right)$. The couplings $A_{h}$ and $A_{j R}$ are 
given by

$$
\begin{aligned}
A_{\frac{1}{2}} & =\sin ^{2} \theta_{W}, A_{-\frac{1}{2}}=-\frac{1}{2}+\sin ^{2} \theta_{W} \\
A_{j R} & =-\sqrt{2} g N_{j 1} \tan \theta_{W} .
\end{aligned}
$$

The amplitude for $\tilde{e}_{L}^{+} \tilde{e}_{L}^{-}$pair production is obtained from Eq. (73) by the replacements

$$
\begin{aligned}
A_{h} & \rightarrow A_{-h} \\
1 \pm(-)^{\bar{h}+1 / 2} & \rightarrow 1 \mp(-)^{\bar{h}+1 / 2} \\
A_{j R} & \rightarrow A_{j L}=\frac{g}{\sqrt{2}}\left(N_{j 2}+N_{j 1} \tan \theta_{W}\right) .
\end{aligned}
$$

The contribution of $t$-channel neutralino exchange to the amplitudes above is easy to understand. For $\tilde{e}_{R}$ pair production, the $t$-channel amplitude contributes only to $\mathcal{M}(1 / 2,-1 / 2)$; for $\tilde{e}_{L}$ pairs, it contributes only to $\mathcal{M}(-1 / 2,1 / 2)$. This follows from the fact that gaugino interactions preserve chirality, and so only the $\tilde{e}_{R} e_{R} \tilde{B}$ and $\tilde{e}_{L} e_{L} \tilde{B}$ couplings are non-vanishing.

The $s$-channel amplitude contributes to both amplitudes $\mathcal{M}(1 / 2,-1 / 2)$ and $\mathcal{M}(-1 / 2,1 / 2)$. In the limit $s \gg m_{Z}^{2}$, and for right-polarized $e^{-}$beams with $h=1 / 2$, one finds

$$
\begin{aligned}
& e^{+} e_{R}^{-} \rightarrow \tilde{e}_{R}^{+} \tilde{e}_{R}^{-} i \mathcal{M}(1 / 2,-1 / 2)=-i C g^{\prime 2} \\
& e^{+} e_{R}^{-} \rightarrow \tilde{e}_{L}^{+} \tilde{e}_{L}^{-} i \mathcal{M}(1 / 2,-1 / 2)=-i C \frac{1}{2} g^{\prime 2}
\end{aligned}
$$

where $C=\lambda e^{i \lambda \phi} \sin \theta \beta_{f}$. In this high-energy limit, the $Z$ mass may be neglected, and the $\gamma$ and $Z$ mass eigenstates may be replaced by the gauge eigenstates $B$ and $W^{0}$. Since $e_{R}$ does not couple to $W^{0}$, only the hypercharge gauge boson contributes, and the amplitudes are proportional to the hypercharge coupling $g^{\prime}$, with hypercharges 1 and $1 / 2$ for $\tilde{e}_{R}$ and $\tilde{e}_{L}$.

This example illustrates the power of beam polarization. By polarizing the beam, we can switch off some Feynman diagrams 'by hand.' One may then isolate particular amplitudes and determine fundamental parameters precisely [56, 57. For example, if we use right-polarized $e^{-}$beams and observe $\tilde{e}_{R}$ and $\tilde{e}_{L}$ pair production with the rates predicted by Eqs. (79) and (80), we can establish the chirality-preserving nature of the supersymmetric gaugino interactions.

For $e^{+} e^{-} \rightarrow \tilde{e}_{L}^{+} \tilde{e}_{R}^{-}$production, the $s$-channel amplitude is absent because gauge interactions preserve chirality. The same is true for $e^{-} e^{-} \rightarrow$ $\tilde{e}_{R(L)}^{-} \tilde{e}_{R(L)}^{-}$, where the $s$-channel amplitude is forbidden by total lepton number conservation. In this case, the $t$-channel amplitude has a new feature: it 
is proportional to the mass of the neutralino exchanged in the $t$-channel, which is required to flip chirality. For example,

$$
\begin{aligned}
e^{+} e^{-} \rightarrow \tilde{e}_{L}^{+} \tilde{e}_{R}^{-} i \mathcal{M}(h, \bar{h}) & =i \sqrt{s} \delta_{\lambda, 0}\left[1+(-)^{\bar{h}-\frac{1}{2}}\right] \\
& \times \sum_{j} \frac{1}{2} m_{\tilde{\chi}_{j}^{0}} \frac{A_{j L} A_{j R}}{t-m_{\tilde{\chi}_{j}^{0}}^{2}} .
\end{aligned}
$$

The existence of the $t$-channel amplitude for $e^{-} e^{-} \rightarrow \tilde{e}^{-} \tilde{e}^{-}$proves that the exchanged $t$-channel particle is a Majorana fermion. Note also that the amplitude is now not proportional to the velocity $\beta$, and so the cross section now grows like $\beta$ near threshold. This behavior was discussed in Sec. (5.2) in the context of threshold mass measurements. Finally, the amplitude is proportional to $M_{1}$ in both of the limits $M_{1} \ll M_{2},|\mu|$ and $M_{1} \gg M_{2},|\mu|$. The total cross section is therefore directly related to fundamental mass parameters.

At high enough beam energies, selectrons will be produced through all mechanisms $\left(\tilde{e}_{L}^{+} \tilde{e}_{L}^{-}, \tilde{e}_{R}^{+} \tilde{e}_{R}^{-}\right.$, and $\left.\tilde{e}_{L}^{ \pm} \tilde{e}_{R}^{\mp}\right)$. All of these processes may result in the signal $e^{+} e^{-}+\not p_{T}$, as discussed above in Sec. (5.1). However, the various signals may often be disentangled, both kinematically, as the energy ranges of the electrons and positrons differ for the different processes, and by comparing the cross sections with various beam polarizations.

Slepton production cross sections have interesting dependences on the fundamental supersymmetry parameters. Fig. 17 shows cross sections for $\tilde{e}_{R}$ pair production in the $\left(M_{1}, \tan \beta\right)$ plane. Here we fix the LSP $\tilde{\chi}_{1}^{0}$ mass. The GUT relation for $M_{1}$ and $M_{2}$ is also assumed. Not surprisingly, there is almost no dependence on $\tan \beta$. This dependence enters only through the neutralino mass matrix in the form of $\cos \beta$ and $\sin \beta$, which are both fairly constant for moderate and large $\tan \beta$. For a completely right-polarized beam, the only state exchanged in the $t$-channel is the Bino. This $t$-channel contribution dominates at the left-hand side of the plot, and then decouples for large $M_{1}$. At the left-hand side of the plot, the LSP is Bino-like, but becomes Higgsino-like on the right. However, the production proceeds through the neutralino with largest $\tilde{B}$ component. The $\tan \beta$ independence of the cross section is even more prominent for the process $e^{-} e^{-} \rightarrow \tilde{e}^{-} \tilde{e}^{-}$, where chirality requires a gaugino mass insertion, which is independent of neutralino mixings. This process also provides sensitivity to even very large $M_{1}$, and may allow for a measurement of the Bino mass even when the Bino is far from kinematically accessible [64, 67.

We have concentrated on selectrons here, as they have many interesting 


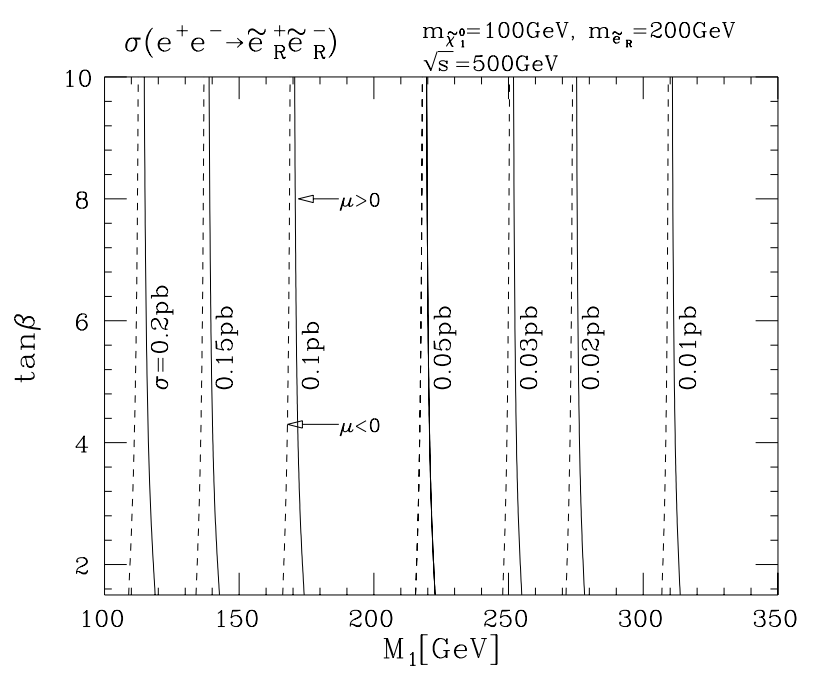

Fig. 17. Contours of $\sigma\left(e^{+} e_{R}^{-} \rightarrow \tilde{e}_{R}^{+} \tilde{e}_{R}^{-}\right)$for $m_{\tilde{e}_{R}}=200 \mathrm{GeV}$ and $\sqrt{s}=500 \mathrm{GeV}$ in the $\left(M_{1}, \tan \beta\right)$ plane 73$]$. At each point in the plane, $\mu$ is chosen so that $m_{\tilde{\chi}_{1}^{0}}=100 \mathrm{GeV}$. Solid lines correspond to a $\mu>0$ solution and dashed lines to $\mu<0$.

properties related to the existence of $t$-channel amplitudes. It should be stressed that smuon and stau studies are also interesting. In particular, stau studies may be very important at the linear collider. The lighter stau is often the lightest scalar superpartner as a result of GUT-scale interactions (see Sec. (4.6)), renormalization group evolution between the GUT and weak scales (see Sec. (4.1)), and left-right mixing (see Sec. (2.5)). Note that staus may also be studied at the LHC, but such studies are significantly complicated by $\tau$ decay to low energy leptons or mesons.

The signal of $\tilde{\tau}$ production is very complicated and will be considered in more detail in Sec. (5.5). Here we merely note that the production amplitude for $e^{+} e^{-} \rightarrow \tilde{\tau}_{1}^{+} \tilde{\tau}_{1}^{-}$in the limit $\sqrt{s} \gg m_{Z}$ for right-polarized electrons is $[74$

$$
i \mathcal{M}(1 / 2,-1 / 2)=-i C g^{\prime 2}\left(\frac{1}{2} \cos ^{2} \theta_{\tilde{\tau}}+\sin ^{2} \theta_{\tilde{\tau}}\right),
$$

where $\theta_{\tilde{\tau}}$ is the stau left-right mixing angle. The measurement of the $\tilde{\tau}$ production cross section for right-polarized electron beams will determine $\theta_{\tilde{\tau}}$. If one finds $\tilde{\tau}_{2}$ in addition to $\tilde{\tau}_{1}$, the full $\tilde{\tau}$ mass matrix may be reconstructed. The reconstruction of the $\tilde{\tau}$ mass matrix will provide yet another opportunities to test the universality of scalar masses at the high scale. In 
addition, the off-diagonal stau mass $m_{L R}^{2}$ is proportional to $\tan \beta$ and $\mu$ as noted in Eq. (22), and the decay distribution also depends on $\tan \beta$, as we will see. Stau studies may therefore provide a rare opportunity to measure $\tan \beta$ for large $\tan \beta$.

\subsection{Lepton Flavor Violation}

As noted in Sec. (2.5), there is no reason for the fermion and sfermion mass matrices to be simultaneously diagonalizable. This implies that the discovery of superpartners will lead to a whole new sector of flavor physics to explore. In the standard model, after the initial discoveries and mass measurements, the focus has naturally turned to measurements of flavor violation, such as in the quark and neutrino sectors. A similar progression may be expected if superpartners are discovered.

At the moment, there is no standard explanation of fermion masses. Recently, our knowledge of neutrino mixing has expanded tremendously, but no compelling theory of flavor has emerged. One might wonder if supersymmetric flavor studies will also lead to a bewildering wealth of data without furthering our fundamental understanding of flavor. While possible, there are important differences in these two cases. In the case of neutrinos, the newly discovered mixings are in principle unrelated to those of the quarks. For example, in theories that attempt to explain flavor through horizontal flavor symmetries, neutrino mixing is dependent on a whole new set of flavor representation assignments. For superpartners, however, the new mixings are tied to the flavor properties of standard model fermions by supersymmetry. For example, standard model particles and their superpartners must be governed by the same flavor symmetries, since they are in the same supermultiplet. In these frameworks, then, superpartners do not introduce additional degrees of freedom, but rather provide new constraints on the same flavor physics governing the standard model particles. For this reason, the careful investigation of superpartner flavor symmetries provides a promising avenue for understanding not only superpartner properties, but also the masses and mixings observed in the standard model.

The full $6 \times 6$ slepton mass matrix of Eq. (18) is very complicated. As a first step, one may begin by neglecting left-right mixing, a reasonable approximation for selectrons and smuons. One can further specialize to two generation mixing and consider, for example, $\tilde{e}_{R}-\tilde{\mu}_{R}$ mixing. A convenient basis to consider is the mass eigenstate basis for both charged leptons and sleptons, in which all mixing is confined to gaugino vertices. Lepton flavor 
violation (LFV) then occurs in slepton pair production through $t$-channel neutralino exchange and in decay vertices $[75,76]$. In simple scenarios where the sleptons decay directly to a neutralino LSP, the resulting signals of flavor-violating slepton pair production at the linear collider are $e^{+} e^{-} \rightarrow$ $\tilde{l}^{+} \tilde{l}^{-} \rightarrow e^{ \pm} \mu^{\mp} \tilde{\chi}_{1}^{0} \tilde{\chi}_{1}^{0}$ and $e^{-} e^{-} \rightarrow \tilde{l}^{-} \tilde{l}^{-} \rightarrow e^{-} \mu^{-} \tilde{\chi}_{1}^{0} \tilde{\chi}_{1}^{0}$.

The probability for flavor-violating decay is

$$
P\left(\tilde{e}_{R} \rightarrow \mu\right)=\frac{1}{2} \sin ^{2} 2 \theta_{R} \frac{\left(\Delta m_{R}^{2}\right)^{2}}{4 m_{R}^{2} \Gamma^{2}+\left(\Delta m_{R}^{2}\right)^{2}},
$$

where $\Delta m_{R}^{2}=m_{1}^{2}-m_{2}^{2}$ and $m_{R}=\left(m_{1}+m_{2}\right) / 2$, with $m_{1}$ and $m_{2}$ the physical slepton masses, and $\theta_{R}$ is the flavor mixing angle. $\Gamma$ is the slepton decay width. If $m_{R} \Gamma \ll \Delta m_{R}^{2}$, the mixing probability has its maximal value of $\left(\sin ^{2} 2 \theta_{R}\right) / 2$; however, for $m_{R} \Gamma \gg \Delta m_{R}^{2}, P$ vanishes, since sleptons decay before they have time to mix.

In the $e^{+} e^{-}$case, flavor violation requires a careful treatment of $t$ channel and $s$-channel interference, as flavor violation is present in $t$-channel diagrams, but absent in $s$-channel processes. However, as with neutrino studies, results are conveniently presented in the $\left(\sin 2 \theta_{R}, \Delta m_{R}^{2}\right)$ plane. In Fig. 18, cross sections for the flavor-violating signal are given, along with the discovery reach of the linear collider. Beam polarization again provides a useful tool to reduce background from processes such as $W^{+} W^{-}$and $e^{+} \nu W^{-}$. Given an integrated luminosity of $50 \mathrm{fb}^{-1}, e-\mu$ flavor violation can be discovered for mixing angles of order $\theta_{R} \sim 0.05$ and mass degeneracies at the $1 \%$ level. For the same luminosity, the $e^{-} e^{-}$mode provides an even better discovery potential, as backgrounds such as $W^{+} W^{-}$are completely prohibited by total lepton number conservation.

Supersymmetric flavor violation is already constrained by low energy data, such as $\mu-e$ conversion and $\mu \rightarrow e \gamma$. In Fig. 18, these constraints are given by straight lines. These are highly dependent on supersymmetry parameters, notably $\tan \beta$, but are suppressed by $\Delta m_{R}^{2} / m_{R}^{2}$ through the supersymmetric analogue of the GIM mechanism. Note that the mass splitting below which LFV is suppressed is set by the slepton width $\Gamma$ for the collider signal, but by the slepton mass $m_{R}$ for low energy constraints. Since $\Gamma \ll m_{R}$, there is a large range of mass splittings in which LFV is suppressed in low energy experiments, but observable at colliders.

At the same time, because the high-energy and low-energy LFV rates have different functional dependences on the mass splitting and mixing angle, simultaneous measurements of LFV in both high- and low-energy experiments will provide complementary information. The combined results may 


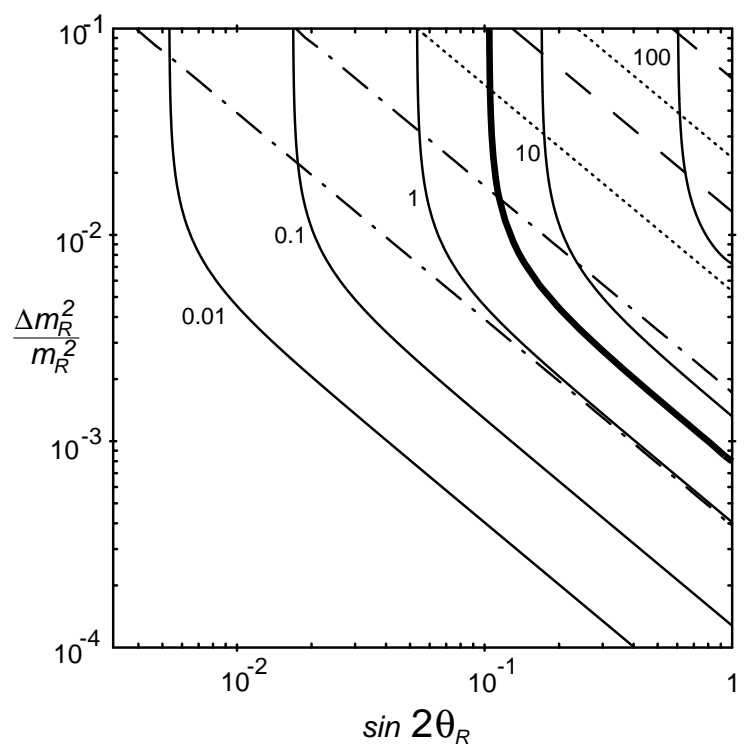

Fig. 18. The solid contours are for $\sigma\left(e^{+} e_{R}^{-} \rightarrow e^{ \pm} \mu^{\mp} \tilde{\chi}_{1}^{0} \tilde{\chi}_{1}^{0}\right)$ in fb for $\sqrt{s}=500 \mathrm{GeV}$, $m_{\tilde{e}_{R}}, m_{\tilde{\mu}_{R}} \approx 200 \mathrm{GeV}$, and $M_{1}=100 \mathrm{GeV}$ 75. The thick contour represents the LFV discovery reach given $50 \mathrm{fb}^{-1}$. Constant contours of $B(\mu \rightarrow e \gamma)=4.9 \times 10^{-11}$ and $2.5 \times 10^{-12}$ are also plotted for degenerate left-handed sleptons with mass $350 \mathrm{GeV}$ and $-(A+\mu \tan \beta) / m_{R}=0$ (dotted), 2 (dashed), and 50 (dot-dashed).

even allow the extraction of mass splittings and mixing angles separately, providing valuable information for attempts to identify the fundamental origin of quark and lepton masses and mixings.

\subsection{Tau Polarization from Stau Decay}

The polarizations of all leptons resulting from slepton decay carry information about the underlying supersymmetry parameters. Typically, of course, these polarizations are unobservable. However, in the case of taus, polarization can be measured at colliders. Here we describe the parameter dependence of the polarization of taus from stau decay and show how this polarization can be reconstructed experimentally.

Tau leptons produced in stau decay have the simple energy distribution of Eq. (69). The $\tau$ decays into $A \nu_{\tau}$, where $A=e \nu_{e}, \mu \nu_{\mu}, \pi, \rho, a_{1}$. The heavy mesons further decay into pions through $\rho^{ \pm} \rightarrow \pi^{ \pm} \pi^{0}$ and $a_{1}^{ \pm} \rightarrow \pi^{ \pm} \pi^{\mp} \pi^{ \pm}, \pi^{ \pm} \pi^{0} \pi^{0}$. The signature of $\tilde{\tau}$ pair production is therefore two acoplanar jets with low multiplicity. 


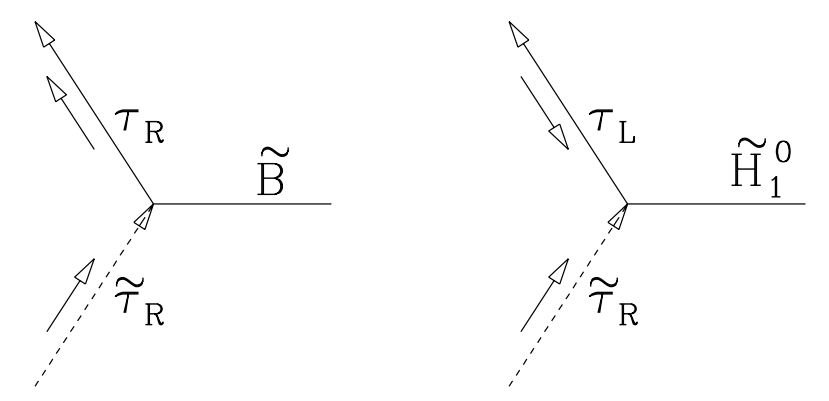

Fig. 19. The flow of chirality in interactions of $\tilde{\tau}_{R}$ with gauginos and Higgsinos.

Because $E_{\tau} \gg m_{\tau}$, the decay products maintain the original direction of the parent $\tau$. However, the total energy of the decay products is substantially reduced since the tau neutrinos escape detection. The $E_{\text {jet }(\tau)}$ distribution is therefore not flat, but decreases to zero at the endpoint, degrading the sharp edge expected in $\tilde{\mu}$ or $\tilde{e}$ pair production. Fits to the total $E_{\text {jet }}$ distribution yield both $m_{\tilde{\tau}}$ and $m_{\tilde{\chi}_{1}^{0}}$, but the sensitivity to $m_{\tilde{\tau}}$ is reduced.

On the positive side, however, this jet energy distribution contains information about the $\tau$ polarization, which in turn depends on the stau and neutralino mass eigenstates. The chirality of a sfermion is preserved in the resulting fermion in gauge, and therefore, gaugino interactions. On the contrary, Higgs, and therefore Higgsino, interactions flip the chirality. This is depicted in Fig. 19, where the arrows indicate the flow of chirality. The two types of interaction also have different couplings, with the $\tau \tilde{\tau} \tilde{H}$ coupling proportional to tau Yukawa coupling $y_{\tau} \propto m_{\tau} / \cos \beta$ and the $\tau \tilde{\tau} \tilde{B}$ and $\tau \tilde{\tau} \tilde{W}^{0}$ couplings proportional to gauge couplings $g_{1}$ and $g_{2}$, respectively.

For a general stau mass eigenstate, in the limit where the lightest neutralino is a pure Bino state, the $\tau$ polarization is given by

$$
P_{\tau}\left(\tilde{\tau}_{1} \rightarrow \tilde{B} \tau\right)=\frac{4 \sin ^{2} \theta_{\tau}-\cos ^{2} \theta_{\tau}}{4 \sin ^{2} \theta_{\tau}+\cos ^{2} \theta_{\tau}}
$$

depending only on $\theta_{\tau}$, as expected. On the other hand, if the neutralino is a mixed object, there is a non-trivial dependence on both gauge and Yukawa couplings. For a general neutralino eigenstate, in the limit of a pure $\tilde{\tau}_{R}$ state, the resulting tau polarization is

$$
P_{\tau}\left(\tilde{\tau}_{R} \rightarrow \tilde{\chi}_{1}^{0} \tau\right)=\frac{\left(g \sqrt{2} N_{11} \tan \theta_{W}\right)^{2}-\left(y_{\tau} N_{13}\right)^{2}}{\left(g \sqrt{2} N_{11} \tan \theta_{W}\right)^{2}+\left(y_{\tau} N_{13}\right)^{2}} .
$$


Because $\theta_{\tilde{\tau}}$ can be measured through the polarization dependence of the stau production cross section, as noted in Sec. (5.3), a measurement of the $\tau$ polarization in this case will provide information on the tau Yukawa coupling and $\tan \beta$. As $y_{\tau}=g m_{\tau} /\left(\sqrt{2} m_{W} \cos \beta\right)$ is comparable to gauge couplings only for large $\tan \beta$, the sensitivity to $\tan \beta$ is high only for large $\tan \beta \gtrsim 10$ when the charginos and neutralinos involved have significant Higgsino components.

The branching fraction of taus to $\rho$ mesons is large, with $B(\tau \rightarrow \rho) \approx$ $23 \%$, and the $\rho$ polarization is mostly longitudinal for $\tau_{R}$ and transverse for $\tau_{L}$. One can thus determine $P_{\tau}$ by measuring $P_{\rho}$. The pion energy distributions in $\rho_{L(T)} \rightarrow \pi^{ \pm} \pi^{0}$ decay are simple functions of $z_{c}=E_{\pi^{ \pm}} / E_{\text {jet }}$, where $E_{\text {jet }}$ is the total energy of the jet to which the $\pi^{ \pm}$belongs. These distributions are

$$
\begin{aligned}
& \frac{d \Gamma\left(\rho_{T} \rightarrow 2 \pi\right)}{d z_{c}} \sim 2 z_{c}\left(1-z_{c}\right)-2 m_{\pi}^{2} / m_{\rho}^{2} \\
& \frac{d \Gamma\left(\rho_{L} \rightarrow 2 \pi\right)}{d z_{c}} \sim\left(2 z_{c}-1\right)^{2},
\end{aligned}
$$

where $\left(1-\beta_{\pi}\right) / 2 \leq z_{c} \leq\left(1+\beta_{\pi}\right) / 2$ and $\beta_{\pi}=\sqrt{1-4 m_{\pi}^{2} / m_{\rho}^{2}}$.

A detailed Monte-Carlo study of tau polarization is done in Ref. 73. In this work, detector granularity is incorporated in the detector simulation, and tracking information is used to subtract the charged track fraction of the energy. The study finds reasonable $\pi, \rho$, and $a_{1}$ separation through the measurement of jet invariant mass, and $\rho$ candidates are used to determine the $\tau$ polarization. Determination of $P_{\tau}$ by measuring $E_{\pi^{+}} / E_{j e t}$ with the error of $\delta P_{\tau}=0.08$ appears possible given $10^{4}$ stau pairs. (See Fig. 20.) However, conclusive results may require a full detector simulation.

\section{Chargino and Neutralino Studies}

\subsection{Signal and Background}

The gaugino mass relations $M_{1}: M_{2}: M_{3} \simeq 1: 2: 7$ are predicted in scenarios with unified gaugino masses at the GUT scale, and also in gaugemediated supersymmetry breaking scenarios. This hierarchy implies that Binos and Winos, or more generally, charginos and neutralinos, are among the lightest superpartners, and so are among the most likely to be produced at the linear collider.

The relevant production processes include

$$
e^{+} e^{-} \rightarrow \tilde{\chi}_{1}^{0} \tilde{\chi}_{1}^{0}, \tilde{\chi}_{1}^{0} \tilde{\chi}_{2}^{0}, \tilde{\chi}_{1}^{ \pm} \tilde{\chi}_{1}^{\mp}, \tilde{\chi}_{2}^{0} \tilde{\chi}_{2}^{0} .
$$



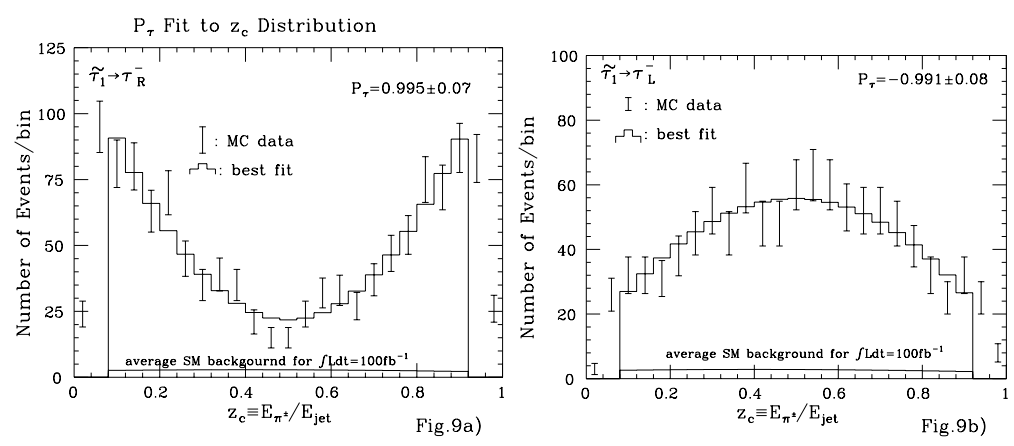

Fig. 20. Distributions in $z_{c}$ for $\rho$ candidates selected from $10^{4} \tilde{\tau}_{1}$ pairs decaying exclusively into $\tau_{R}$ (left) and $\tau_{L}$ (right), together with the best fit histograms 73 .

In favorable scenarios where $|\mu| \sim M_{1}, M_{2}$, such as the focus point models discussed in Sec. (4.2), the $\tilde{\chi}_{2}^{ \pm}, \tilde{\chi}_{3}^{0}$, and $\tilde{\chi}_{4}^{0}$ states may also be produced, allowing access to the entire neutralino and chargino systems. Neutralino production proceeds through $s$-channel $Z$ boson exchange and $t$-channel $\tilde{e}$ exchange. Charginos are produced through $s$-channel exchange of $\gamma$ and $Z$ and $t$-channel $\tilde{\nu}_{e}$ exchange.

In many models, the lightest neutralino $\tilde{\chi}_{1}^{0}$ is stable and therefore invisible. In this case, the first process of Eq. (87) is unobservable. (It is a frustrating fact that thousands of neutralinos may already have been produced in colliders without our knowledge.) The heavier states typically decay visibly, however, with decay modes such as

$$
\begin{aligned}
\tilde{\chi}_{1}^{+} & \rightarrow W^{+} \tilde{\chi}_{1}^{0} \\
& \rightarrow \tilde{\tau}^{+} \nu, \tilde{l}^{+} \nu, \tilde{\nu} l^{+} \\
& \rightarrow \bar{q}^{\prime} q \tilde{\chi}_{1}^{0}, l^{+} \nu \tilde{\chi}_{1}^{0} \\
\tilde{\chi}_{2}^{0} & \rightarrow h \tilde{\chi}_{1}^{0}, Z \tilde{\chi}_{1}^{0} \\
& \rightarrow \tau \tilde{\tau}, l \tilde{l} \\
& \rightarrow l l \tilde{\chi}_{1}^{0}, \nu \bar{\nu} \tilde{\chi}_{1}^{0}, \bar{q} q \tilde{\chi}_{1}^{0} .
\end{aligned}
$$

The signal of chargino pair production is therefore missing momentum accompanied by $4 j, 2 j+l$, or $l^{+} l^{\prime-}$. Neutralino pair signals are similar, but with each charged lepton replaced by a same flavor lepton pair. Note that if the lightest neutralino is stable, the second lightest neutralino is also invisible if it decays to $\nu \bar{\nu} \tilde{\chi}_{1}^{0}$.

As in the case of sleptons, the dominant backgrounds for chargino and neutralino events are again various standard model gauge boson processes, such as $W W$ and $Z Z$. These may be removed with cuts similar to those 
used for slepton production. Note, however, that Wino-like states couple only to left-handed (s)electrons. In the case of pair production of Wino-like states, then, a right-polarized electron beam reduces both the signal and backgrounds.

\subsection{Masses and Polarized Cross Sections}

Chargino production is among the most well-studied supersymmetric processes, and we will concentrate on charginos here, although neutralinos also provide many insights. The fundamental supersymmetry parameters entering the chargino mass matrix of Eq. (27) are $\mu, M_{2}$, and $\tan \beta$. These determine not only the chargino masses, but also the chargino mixing matrices $U_{i j}$ and $V_{i j}$ of Eq. (28). The production cross section also depends on the sneutrino mass that enters through the $t$-channel process. The chargino pair production cross section is therefore determined by four supersymmetry parameters:

$$
\mu, M_{2}, \tan \beta, m_{\tilde{\nu}_{e}} .
$$

In addition to these, the observables of chargino events depend on the parameters entering chargino decay. These include $M_{1}$ and other sparticle masses. Note that chargino production and decay are intertwined through spin correlations, and so cannot be treated separately in principle.

The flexibility of the linear collider provides the possibility of disentangling all of these parameters even if only one chargino state is kinematically accessible [57, 77, 78, 79. First, for center-of-mass energies reasonably far above threshold, the impact of decay parameters on many observables may be reduced to low levels. Second, beam polarization allows a variety of cross section measurements with different dependences on the fundamental parameters. Among the most important quantities are

$$
m_{\tilde{\chi}_{1}^{+}}, \sigma_{R}, A_{R}^{F B}, \sigma_{L}, A_{L}^{F B},
$$

where $\sigma$ and $A^{F B}$ are the total cross section and forward-backward asymmetry for chargino production, and the subscripts $L$ and $R$ denote left- and right-polarized electron beams. Each of these quantities has an interesting and unique dependence on the fundamental parameters.

The chargino mass $m_{\tilde{\chi}_{1}^{+}}$depends in principle on $M_{2},|\mu|$, and $\tan \beta$. However, when the off-diagonal entries are small compared to the diagonal entries, as is often the case, $m_{\tilde{\chi}_{1}^{+}}$provides essentially a direct measurement of $M_{2}$ or $|\mu|$, whichever is smaller. The chargino mass can be measured by kinematic endpoints through analyses similar to those described in 


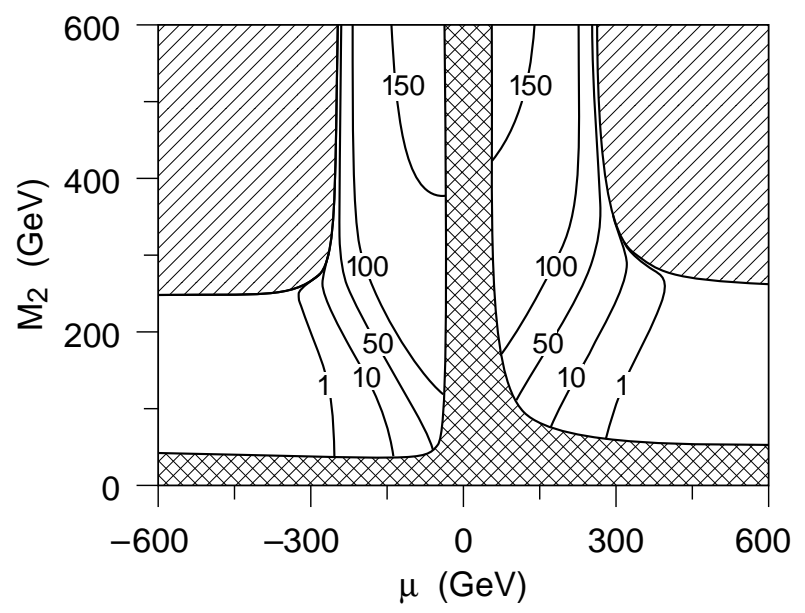

Fig. 21. Contours of $\sigma\left(e^{+} e_{R}^{-} \rightarrow \tilde{\chi}_{1}^{+} \tilde{\chi}_{1}^{-}\right.$) (in fb) in the $\left(\mu, M_{2}\right)$ plane for fixed $\tan \beta=$ 4 and $\sqrt{s}=500 \mathrm{GeV}$. The cross-hatched region is excluded by current bounds, and charginos are kinematically inaccessible in the hatched region [77].

Sec. (5.2). It may also be measured very precisely through threshold scans, since the cross section is proportional to $\beta_{\tilde{\chi}_{1}^{+}}$, as discussed in Sec. (5.2).

Although the chargino cross section has both $s$-channel and $t$-channel contributions in general, the $t$-channel sneutrino contribution is eliminated in the limit of purely right-polarized electron beams. The right-polarized cross section therefore contains only $s$-channel contributions mediated by $\gamma$ and $Z$ gauge bosons. As discussed in Sec. (5.3), in the high energy limit $\sqrt{s} \gg m_{Z}$ where the $Z$ mass is negligible, the exchanged gauge bosons may be replaced by the gauge eigenstates $B$ and $W^{0}$ to an excellent approximation. The $W^{0}$ contribution is absent for right-polarized electrons, while $B$ exchange is absent for Wino-like charginos. The right-polarized cross section $\sigma_{R}$ is therefore highly suppressed for Winos and is a sensitive measure of the Higgsino content of the chargino. This can be seen in Fig. 21. For $M_{2} \ll|\mu|$ where the lighter chargino is Wino-like, $\sigma_{R}$ is all but absent, while for $M_{2} \gg|\mu|$, where the lighter chargino is Higgsino-like, $\sigma_{R}$ is large. Note that $\sigma_{R}$ becomes near maximal even very close to threshold (the hatched region) as a result of the $\beta$ threshold behavior. $A_{R}^{F B}$ is also sensitive to chargino mixing, with a dependence different from $\sigma_{R}$. The right-polarized quantities $\sigma_{R}$ and $A_{R}^{F B}$ therefore provide information on chargino mixing that is highly complementary to the information provided by $m_{\tilde{\chi}_{1}^{+}}$. 


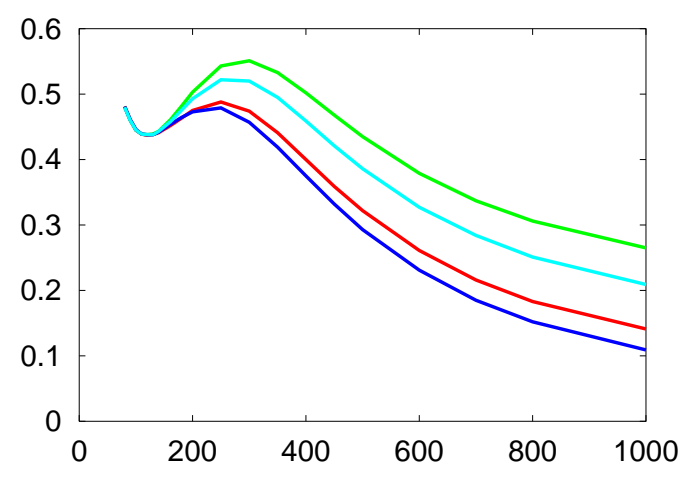

Fig. 22. The forward-backward asymmetry of the final state electron from chargino decay in chargino pair events as a function of the sneutrino mass (in GeV) 79. The curves are for $m_{\tilde{e}_{L}}=130 \mathrm{GeV}, 150 \mathrm{GeV}, 200 \mathrm{GeV}$, and $m_{\tilde{e}_{L}}^{2}=m_{\tilde{\nu}_{e}}^{2}-m_{W}^{2} \cos 2 \beta$, from above. The other underlying parameters are fixed to $M_{2}=152 \mathrm{GeV}, \mu=316 \mathrm{GeV}$, $\tan \beta=3$, and $M_{1}=79 \mathrm{GeV}$, and the center-of-mass energy is $\sqrt{s}=500 \mathrm{GeV}$, with beam polarizations $P_{e^{-}}=-0.85$ and $P_{e^{+}}=-0.6$.

Finally, the left-polarized quantities $\sigma_{L}$ and $A_{L}^{F B}$ are sensitive to the mass of the exchanged sneutrino. The $t$-channel amplitude is proportional to $1 /\left(t-m_{\tilde{\nu}_{e}}^{2}\right)$ where $t=m_{\tilde{\chi}_{1}^{+}}-\left(1-\beta_{\tilde{\chi}_{1}^{+}} \cos \theta\right) s / 2$ and $\theta$ is the angle between the $\tilde{\chi}_{1}^{-}$momentum and the electron beam. The $s$ - and $t$-channel contributions interfere destructively, and $\sigma_{L}$ provides a measurement of $m_{\tilde{\nu}_{e}}$ even when the sneutrino mass is several hundreds of $\mathrm{GeV}$ and sneutrinos are too heavy to be produced directly. In addition, the forward-backward asymmetry is sensitive to the sneutrino mass. This asymmetry may be transferred to the decay products. For example, in Fig. 22, the forwardbackward asymmetry of the final state $e$ in chargino events is shown as a function of $m_{\tilde{\nu}_{e}}$ for various $m_{\tilde{e}_{L}}$, assuming a left-polarized electron beam. Assuming the MSSM $m_{\tilde{\nu}_{e}}-m_{\tilde{e}_{L}}$ splitting relation of Eq. 25), $A_{L}^{F B}$ provides another measurement of $m_{\tilde{\nu}_{e}}$. Alternatively, one can check the validity of the slepton splitting relation using the combined measurements.

Chargino studies therefore provide a rich arena for supersymmetry studies. Many important implications follow from such studies. For example, if the charginos are determined to be Wino-like, the chargino and neutralino masses become highly correlated with $M_{2}$ and $M_{1}$, respectively, allowing a model-independent measurement of these parameters and providing a test of GUT or gauge-mediated predictions. On the other hand, if they are de- 
termined to have significant Wino-Higgsino mixing, upper bounds on the heavier chargino and neutralinos may be obtained. Chargino studies may also determine, or set upper bounds on, sneutrino masses even when sneutrinos are far beyond the kinematic reach of the collider, providing another target energy for future supersymmetry searches.

\subsection{CP Violation}

Because the linear collider allows one to overconstrain the chargino system, as discussed above, it also allows one to explore additional degrees of freedom assumed absent in the simplest models. For example, one may explore supersymmetric sources of CP violation, or even the basic supersymmetry relations between particle and sparticle couplings. We discuss the former here and the latter in Sec. (7).

Many supersymmetric parameters are in general complex. For example, the gaugino and Higgsino mass parameters are, in general,

$$
\mu=|\mu| e^{i \phi_{\mu}}, M_{i}=\left|M_{i}\right| e^{i \phi_{i}} .
$$

As we already discussed in Sec. (3.4), CP-violating phases of supersymmetry parameters are strongly constrained by neutron and electron EDMs. Large $\mathcal{O}(1)$ phases therefore require fine-tuning among parameters 80]. However, baryogenesis requires some source of $\mathrm{CP}$ violation beyond the standard model, and it is natural to ask how $\mathrm{CP}$ violation in supersymmetry may be discovered. to $^{2}$

The CP phases of supersymmetry parameters may be measured through their impact on CP-conserving quantities such as masses and decay distributions. For example, allowing the $\mu$ parameter to be complex, the two chargino masses are given by 78

$$
m_{\tilde{\chi}_{1,2}^{ \pm}}^{2}=\frac{1}{2}\left[M_{2}^{2}+|\mu|^{2} 2 m_{W}^{2} \mp \Delta_{C}\right],
$$

where

$$
\begin{aligned}
\Delta_{C}^{2}= & \left(M_{2}^{2}-|\mu|^{2}\right)^{2}+4 m_{W}^{4} \cos ^{2} 2 \beta+4 m_{W}^{2}\left(M_{2}^{2}+|\mu|^{2}\right) \\
& +8 m_{W}^{2} M_{2}|\mu| \sin 2 \beta \cos \phi_{\mu} .
\end{aligned}
$$

\footnotetext{
${ }^{\mathrm{d}}$ Large CP-violating phases are also motivated by string theory, where all couplings, including the standard model Yukawa couplings, are expected to result from the dynamical condensation of moduli fields. If soft supersymmetry-breaking terms arise from the same source, large phases in soft supersymmetry parameters are also expected.
} 

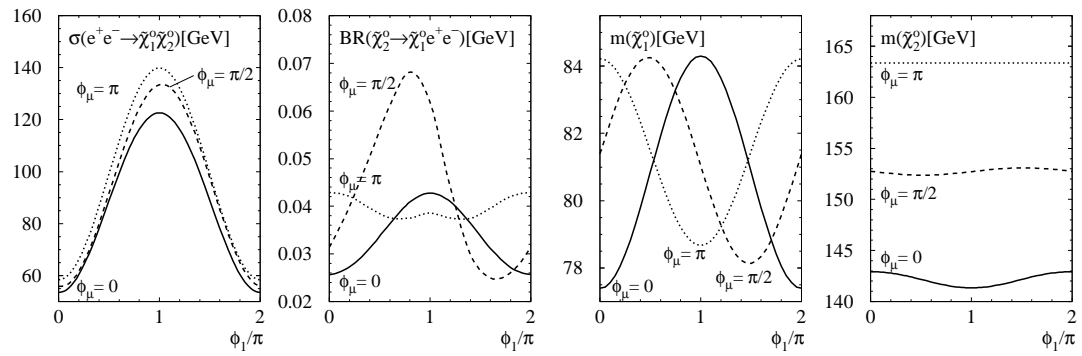

Fig. 23. The dependence of various observables on CP-violating phases for the $\mu$ parameter and Bino mass, assuming magnitudes of the supersymmetry parameters as given in minimal supergravity with $m_{0}=100 \mathrm{GeV}, M_{1 / 2}=200 \mathrm{GeV}, A_{0}=0, \tan \beta=4$ and $\mu>0[32]$.

If the full chargino system is available and the two chargino masses and two mixing angles $\phi_{+}$and $\phi_{-}$, suitably modified to diagonalize the complex chargino mass matrix [78], are constrained, deviations of $\phi_{\mu}$ from zero may be observed. CP-violating phases also distort the neutralino mass spectrum 81.

The dependence of various CP-conserving observables on the phases of $\mu$ and $M_{1}$ are shown in Fig. 23. Significant variations are clearly possible. In these figures, however, the magnitudes of all parameters are held fixed. It is therefore not clear whether the effects of CP-violating phases may be mimicked simply by suitable adjustments of real parameters. To answer this question, a CP-violating scenario was studied in Ref. 82]. The predicted values for three cross sections, $\sigma\left(\tilde{\chi}_{1}^{0} \tilde{\chi}_{2}^{0}\right), \sigma\left(\tilde{\chi}_{2}^{0} \tilde{\chi}_{2}^{0}\right)$, and $\sigma\left(\tilde{\chi}_{1}^{ \pm} \tilde{\chi}_{1}^{\mp}\right)$, and three masses, $m_{\tilde{\chi}_{1}^{0}}, m_{\tilde{\chi}_{2}^{0}}$, and $m_{\tilde{\chi}_{1}^{ \pm}}$were then determined. The measured values of these observables were then assumed to lie in Gaussian distributions around these central values, where the Gaussian widths were chosen to simulate realistic experimental resolutions and statistical errors. 10,000 'pseudo-data sets' were formed by choosing values of these observables within these distributions, and for each pseudo-data set, the best fit underlying supersymmetry parameters were determined.

Fig. 24 shows the results of this study. The input model parameters are shown as arrows in the figures. The left panel shows best fit values of $M_{1}$ and $\phi_{1}$ for the 10,000 data sets, assuming $\tan \beta=4$ fixed to its underlying value (dark points) and including $\tan \beta$ among the fitted parameters (light green points). The $\phi_{1}$ distribution is summarized in the right-hand panel. As can be seen, the hypothesis of a vanishing phase is strongly disfavored. In these examples, the underlying value of $|\mu|=310 \mathrm{GeV}$ is assumed known 

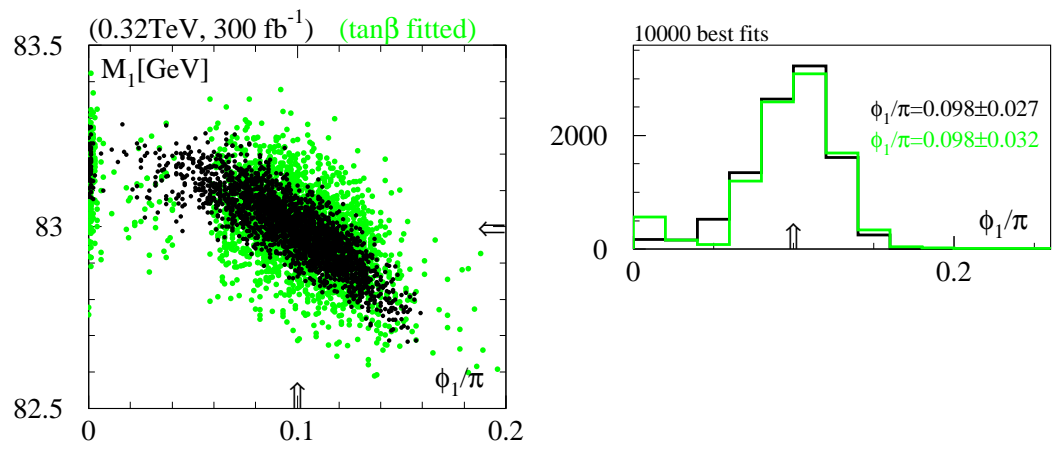

Fig. 24. The best fit values of $M_{1}$ and $\phi_{1}$ given measurements of three cross sections, $\sigma\left(\tilde{\chi}_{1}^{0} \tilde{\chi}_{2}^{0}\right), \sigma\left(\tilde{\chi}_{2}^{0} \tilde{\chi}_{2}^{0}\right)$, and $\sigma\left(\tilde{\chi}_{1}^{ \pm} \tilde{\chi}_{1}^{\mp}\right)$, and three masses, $m_{\tilde{\chi}_{1}^{0}}, m_{\tilde{\chi}_{2}^{0}}$, and $m_{\tilde{\chi}_{1}^{ \pm}}$[32]. The effects of experimental resolution and finite statistics have been included (see text). The parameter $\tan \beta$ is either fixed to its underlying value (dark) or included among the fitted parameters (light green). The right-hand panel summarizes the distribution of best-fit $\phi_{1}$ phases.

from chargino studies. However, uncertainties of $5 \mathrm{GeV}$ in the $\mu$ parameter do not alter these conclusions.

$\mathrm{CP}$ violation may also be measured directly through manifestly CPviolating observables 78 . Such observables are required to be sensitive to the polarization of charginos and neutralinos relative to the plane of production. These observables typically require production of heavy charginos and neutralinos, as in $\tilde{\chi}_{1}^{ \pm} \tilde{\chi}_{2}^{\mp}$ and $\tilde{\chi}_{i}^{0} \tilde{\chi}_{j}^{0}(i \neq j)$ events, and are challenging, as the dominant $\mathrm{CP}$-even contribution becomes a background for the extraction of a small CP-odd component. However, measurements of $\mathrm{CP}$ violation in this way are possible, and such observables could provide direct and unambiguous evidence for supersymmetric $\mathrm{CP}$ violation.

\section{Testing Supersymmetry}

Newly discovered particles need not conform to our preconceived expectations - the $\mu-\pi$ and $c-\tau$ puzzles are well-known cautionary tales. A doubling of the standard model spectrum is often thought to be the smoking gun signal of supersymmetry. However, there is no guarantee that all superpartners will be discovered together. In addition, even if all of the appropriate degrees of freedom are discovered, the new physics need not be supersymmetric.f

${ }^{\mathrm{e}}$ For example, see Ref. [33] for an extra-dimensional model where KK modes provide a new particle spectrum identical to that predicted by supersymmetry. In addition, the 
Can new particles be identified as supersymmetric through incisive tests? Also, if some superpartners are missing, can we constrain their properties, much as precision electroweak data constrained the mass of the top quark previously and constrains the Higgs boson mass now?

It turns out that these two questions may both be answered by testing purely supersymmetric relations. We have already noted in Secs. (2.2) and 2.3) that the gaugino-sfermion-fermion and Higgsino-sfermion-fermion couplings are determined by the gauge and Yukawa couplings of the standard model, respectively. Tests of the equivalence of these couplings are therefore model-independent tests of supersymmetry [77. At the same time, measurements of small deviations from these identities are measurements of supersymmetry breaking, and so constrain soft parameters and the superparticle spectrum $73,84,85,86,87,88,89$.

\subsection{Verifying Supersymmetry}

Exact supersymmetry relates both dimensionful and dimensionless couplings. The dimensionful relations, such as the equivalence of the electron and selectron masses, must be broken, and the new contributions are the model-dependent soft supersymmetry-breaking terms of Sec. (2.4). In contrast, the relations between dimensionless couplings are preserved in all attractive models, as these relations are required if the gauge hierarchy is to be preserved. They therefore provide model-independent predictions that may be exploited to confirm that newly-discovered particles are indeed superpartners.

How well may the dimensionless supersymmetric identities be tested at the linear collider? To quantify this, we may treat the standard model and supersymmetric couplings as independent parameters, and then determine how well the supersymmetric couplings may be determined. These new couplings must be determined along with all of the usual unknown supersymmetry parameters, and the introduction of a yet another degree of freedom implies that many independent measurements of a given reaction are necessary. Testing supersymmetry in this model-independent way therefore makes full use of the flexibility and potential of linear colliders.

Tests of supersymmetry are possible with many different superpartners and many different coupling relations. As an example, consider $\tilde{e}_{R}$ pair

colored KK particles are heavier than the electroweak ones, and even the missing energy signals predicted in many supersymmetry frameworks are mimicked by the existence of a stable weakly-interacting lightest KK particle. 
production. The $t$-channel neutralino exchange process depends on the Bino coupling $g_{\tilde{B} \tilde{e}_{R} e_{R}}$. To investigate the level of sensitivity to this coupling, we define the parameter

$$
Y_{\tilde{B}} \equiv \frac{g_{\tilde{B} \tilde{e}_{R} e_{R}}}{\sqrt{2} g^{\prime}}
$$

Supersymmetry predicts $Y_{\tilde{B}}=1$. In the limit $m_{Z} \ll M_{1},|\mu|$ and assuming $100 \%$ right-handed electron beams, the amplitude for $\tilde{e}_{R}$ pair production is approximately

$$
\mathcal{M} \propto \beta_{\tilde{e}}\left[1-\frac{4 Y_{\tilde{B}}^{2}}{1-2 \cos \theta \beta_{\tilde{e}_{R}}+\beta_{\tilde{e}_{R}}^{2}+4 M_{1}^{2} / s}\right],
$$

where the first and second terms come from $s$ - and $t$-channel processes, respectively. We may therefore constrain both $Y_{\tilde{B}}$ and $M_{1}$ by measuring the differential cross section $d \sigma / d \cos \theta$.

To determine the differential cross section, we must know the slepton production angle. This is, of course, not directly observable. However, in any given event, we may determine it up to a two-fold ambiguity [57]. The angle between an observed lepton and its parent slepton is determined by the lepton energy through Eq. (69). The slepton direction then lies on a cone with known opening angle centered on the lepton direction. The direction of each slepton may be constrained in this way; because the slepton pair must be produced back-to-back, we find two possible solutions for the slepton production angle in each event, as shown in Fig. 25. The distribution of the wrong solution turns out to be more or less flat, and so the combined distribution plotted in Fig. 25 allows one to determine the sparticle angular distribution in a statistical manner.

Given the differential cross section for selectron pair production, as well as kinematic endpoint information to constrain $m_{\tilde{e}_{R}}$ and $m_{\tilde{\chi}_{1}^{0}}$, one may then constrain $Y_{\tilde{B}}$. The results of such an analysis are given in Fig. 26, where we see that $Y_{\tilde{B}}$ may be determined at the $2 \%$ level for integrated

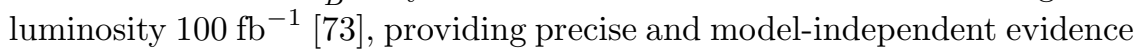
that the produced scalar particle is indeed a selectron. Constraints on $Y_{\tilde{B}}$ of even higher precision are possible from $e^{-} e^{-} \rightarrow \tilde{e}_{R}^{-} \tilde{e}_{R}^{-}$production [85], and the equivalent parameters for the $\mathrm{SU}(2)$ and $\mathrm{SU}(3)$ couplings may also be constrained by chargino and squark studies 85 and also through studies of triple gauge vertices [89]. An exhaustive list of observables with the potential for testing supersymmetry is given in Ref. 84 . 

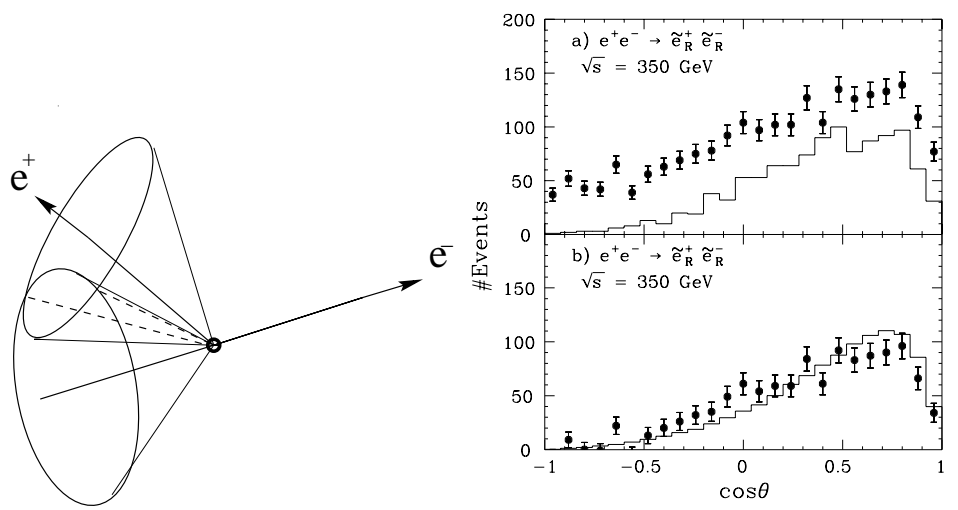

Fig. 25. Left: Reconstruction of the slepton production angle 57. Each parent slepton's direction lies on a cone around the observed lepton momentum. Since the sleptons are produced back-to-back, the slepton directions are then determined up to a two-fold ambiguity to lie along the dashed lines. The cone for $\tilde{e}^{-}$solution has been inverted in the figure. The dashed lines represent the solutions for the $\tilde{e}^{+}$direction. Right: Angular distribution of $e^{+} e^{-} \rightarrow \tilde{e}_{R}^{+} \tilde{e}_{R}^{-}$reconstructed from the final state $e^{+} e^{-}$for underlying parameters $\sqrt{s}=350 \mathrm{GeV}, \tilde{e}_{R}=142 \mathrm{GeV}$, and $\tilde{\chi}_{1}^{0}=118 \mathrm{GeV}$ [57]. The data points with error bars show angular distributions with (a) both right and wrong solutions and (b) with the wrong solution distribution, assumed flat, subtracted (see text). The histograms are the distribution of correct solutions (a) after and (b) before selection cuts.

\subsection{Super-oblique Parameters}

So far we have been discussing the possibility of testing supersymmetry by verifying dimensionless coupling identities. These identities are exact at tree-level. At the loop-level, however, even these relations receive corrections [90]. The breaking of dimensionless relations is called hard supersymmetry breaking [91.

These corrections may be understood by analogy to the oblique corrections [92] of the standard model. In the standard model, SU(2) multiplets with custodial $\mathrm{SU}(2)$-breaking masses, such as the $(t, b)$ multiplet, induce splittings in the couplings of the $(W, Z)$ vector multiplet at the quantum level. Similarly, in supersymmetric models, supermultiplets with soft supersymmetry-breaking masses, such as the $(\tilde{f}, f)$ supermultiplets, induce splittings in the couplings of the (gauge boson, gaugino) vector supermultiplet at the quantum level. This analogy can be made very precise 84, 86. 87. Corrections to hard supersymmetry relations are therefore called super-oblique corrections, and the splittings are typically written in terms of super-oblique parameters. 


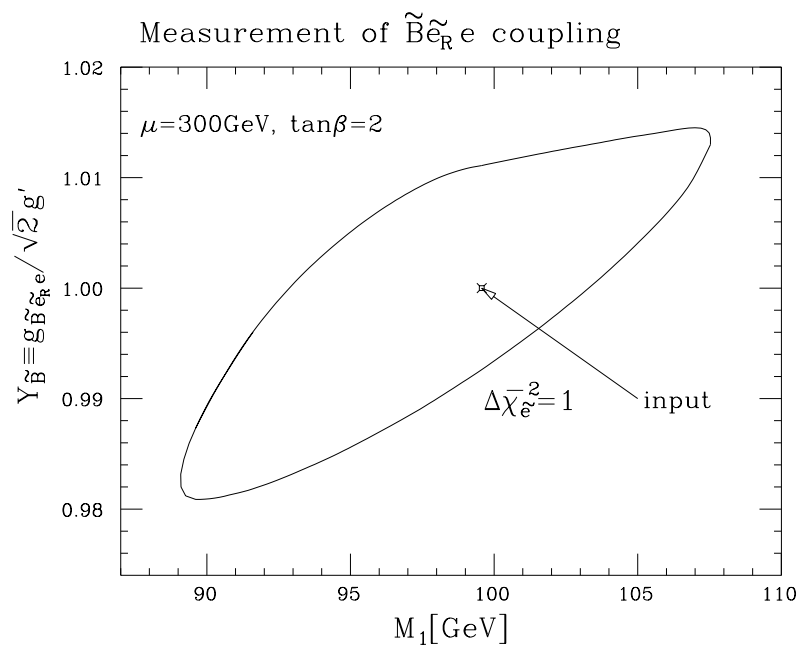

Fig. 26. $\Delta \bar{\chi}_{\tilde{e}}^{2}=1$ contour in the $M_{1}-Y_{\tilde{B}}$ plane for integrated luminosity $100 \mathrm{fb}^{-1} 73$. $\Delta \chi_{\tilde{e}}^{2}=1$ is defined by constraints from electron energy and differential cross sections measurements and is roughly equivalent to a significance of $\Delta \chi^{2}=1$. Input values are $m_{\tilde{e}_{R}}=200 \mathrm{GeV}, \mu=300 \mathrm{GeV}, M_{1}=99.57 \mathrm{GeV}$, and $\tan \beta=2$.

A enlightening viewpoint is to consider the evolution of dimensionless couplings from some high scale in these theories. Above the scale of superpartner masses, the theory is completely supersymmetric, and so the gaugino and gauge couplings remain identical. However, at scales below some sparticle mass, the effective theory is not supersymmetric, and so the supersymmetry relations get corrected. If there are still sparticles in this effective theory, they may be produced at colliders, and their couplings will show evidence of the heavy superpartners.

This viewpoint makes obvious an interesting fact: the super-oblique parameters are non-decoupling. The heavier the decoupled superparticles are, the longer the couplings evolve in the non-supersymmetric theory and the greater their splitting. Measurements of super-oblique parameters at the linear collider therefore provides a method for probing superpartner masses at arbitrarily high scales. Super-oblique parameter measurements are particularly relevant in models with large hierarchies in the supersymmetry spectrum. Such hierarchies are certainly possible, and possibly even favored, given the constraints discussed in Sec. (3), and may be found in models with heavy colored superpartners, focus point supersymmetry, or 
the superheavy supersymmetry scenarios of Sec. (4.3).

For concreteness, let us consider models with

$$
M_{3}, m_{\tilde{q}} \gg m_{\tilde{l}}, M_{2}, M_{1} .
$$

Characterizing the superparticle mass scale with two parameters, $M_{\tilde{Q}}$ for all the heavy states, and $M_{\tilde{L}}$ for all the light states, we find 84]

$$
\begin{gathered}
\tilde{U}_{1} \equiv Y_{\tilde{B}}-1=\frac{11}{5} \frac{g_{1}^{2}}{16 \pi^{2}} \ln \frac{M_{\tilde{Q}}}{M_{\tilde{L}}} \\
\tilde{U}_{2} \equiv Y_{\tilde{W}}-1=3 \frac{g^{2}}{16 \pi^{2}} \ln \frac{M_{\tilde{Q}}}{M_{\tilde{L}}} .
\end{gathered}
$$

where $\tilde{U}_{1}$ and $\tilde{U}_{2}$ are the super-oblique parameters for the $\mathrm{U}(1)$ and $\mathrm{SU}(2)$ gauge groups. Both of these may be measured at linear colliders. For $M_{\tilde{Q}} / M_{\tilde{L}} \sim 10, \tilde{U}_{2} \approx 2.0 \%$ and $\tilde{U}_{1} \approx 0.7 \%$, leading to enhancements of $t$-channel contributions of about $8.0 \%$ and $2.8 \%$, respectively. Given the results described above, such deviations are certainly observable at the linear collider. Non-vanishing super-oblique parameters are signals of supersymmetry breaking, and precise measurements could even constrain the mass scale of superpartners that are far beyond the reach of colliders.

The super-oblique corrections are, of course, just a subset of all radiative corrections. They may be particularly large, given the decoupling picture leading to the $\log M_{Q}$ corrections discussed above. However, additional radiative corrections appear in all supersymmetry processes. Radiative corrections to masses have been presented in Ref. [93]. For some production and decay processes, the full one-loop correction is also available 94. If supersymmetry is discovered, the full radiative analysis of all available processes will become important in precision supersymmetric physics, just as it was for precision electroweak physics. These studies will be important also for global fits and extrapolation to higher mass scales, which we will discuss below in Sec. (9).

\section{Determining the Scale of Supersymmetry Breaking}

If supersymmetry is discovered, the mediation of supersymmetry breaking will be investigated in detail by measuring the soft supersymmetry-breaking terms, as we have discussed. Intimately connected to the mediation mechanism, however, is the mechanism of supersymmetry breaking. As a first step, one would like to know the scale of supersymmetry breaking $F_{\mathrm{DSB}}$ discussed in Sec. (何). This scale determines both the gravitino mass and 
its couplings. In frameworks such as supergravity, where the scale of supersymmetry breaking is high, the gravitino is decoupled from collider phenomenology, and so colliders cannot provide much information about the supersymmetry-breaking scale, other than to provide lower bounds.

In low-scale supersymmetry-breaking scenarios, including the gaugemediated models discussed in Sec. (4.4), however, standard model superpartners may decay to gravitinos on observable scales. This decay provides a window on the gravitational sector of supersymmetry, allowing a quantitative measurement of the scale of supersymmetry breaking, and possibly providing information about the gravitino mass $m_{3 / 2}$, the messenger scale $M_{\text {mess }}$, and so on.

The robust prediction of gauge-mediated models is the existence of a very light gravitino LSP with significant couplings to standard model particles. In these scenarios, standard model superpartners may decay into gravitinos in collider detectors. The decay length for this decay was given in Sec. (4.4). More precisely, for a neutralino NLSP, the decay length for $\tilde{\chi}_{1}^{0} \rightarrow \tilde{G} \gamma$ is

$$
L \simeq 0.10 \mathrm{~mm} \frac{1}{\kappa_{\gamma}^{2}}\left[\frac{100 \mathrm{GeV}}{m_{\tilde{\chi}_{0}^{1}}}\right]^{5}\left[\frac{\sqrt{F_{\mathrm{DSB}}}}{10^{5} \mathrm{GeV}}\right]^{4}\left[\frac{E_{\tilde{\chi}_{0}^{1}}^{2}-m_{\tilde{\chi}_{0}^{1}}^{2}}{m_{\tilde{\chi}_{0}^{1}}^{2}}\right]^{\frac{1}{2}},
$$

where $\kappa_{\gamma}=\left|N_{11} \cos \theta_{W}+N_{12} \sin \theta_{W}\right|$. For typical parameters, this is a macroscopic distance. The NLSP's decay products therefore typically do not point back to the interaction point. Depending on what superpartner is the NLSP, we would then see the following unusual signals at the linear collider:

$$
\begin{aligned}
& e^{+} e^{-} \rightarrow \tilde{l}^{+} \tilde{l}^{-} \rightarrow l l \tilde{G} \tilde{G}+\text { displaced vertices } \\
& e^{+} e^{-} \rightarrow \chi_{1}^{0} \chi_{1}^{0} \rightarrow \gamma \gamma \tilde{G} \tilde{G}+\text { displaced vertices }
\end{aligned}
$$

These signals are spectacular, and may be used to differentiate supersymmetry from backgrounds.]

The signals of Eq. (100) have been studied carefully in Ref. 95. For slepton NLSPs with decay lengths longer than $\sim 10 \mu \mathrm{m}$, the $\tilde{l}$ momentum can be measured by the inner tracking detector. The NLSP lifetime can then be reconstructed with relative ease. In conjunction with NLSP mass measurements, $F_{\mathrm{DSB}}$ will determined to within a few percent.

${ }_{\mathrm{f}}^{\mathrm{f}}$ ote that displaced vertices are particularly helpful at the LHC. For example, given displaced vertices, one can reconstruct the masses of various sparticles, such as $\tilde{\chi}_{3}^{0}$ and $\tilde{\chi}_{4}^{0}$, which might not be accessible in supergravity scenarios 71 . 
The case of neutralino NLSPs is more difficult, but still promising. The linear collider calorimeter has some angular resolution, and will measure decay lengths between $5 \mathrm{~cm}$ and $2 \mathrm{~m}$ with a statistical precision of a few percent. For decay lengths above $\sim 10 \mu \mathrm{m}$ but below $10 \mathrm{~cm}$, three-body decays $\tilde{\chi}_{1}^{0} \rightarrow f \bar{f} \tilde{G}$ may be used to reconstruct the vertex. The branching fraction for such decays is typically a few percent. Finally, for very long decay lengths of the order of $10 \mathrm{~m}$, one can compare the number of 2 photon events to the number of 1 photon events, where, in the latter case, one of the two NLSPs decays outside the detector. This may be sensitive to supersymmetry-breaking scales $\sqrt{F_{\mathrm{DSB}}}$ as large as $2000 \mathrm{TeV}$. In this last case, high luminosity is of great importance.

\section{Extrapolation to the Planck Scale}

In Secs. (5) and (6), we saw numerous examples in which the weak-scale parameters of the MSSM can be determined in a model-independent manner. As described in Sec. (四), these soft supersymmetry-breaking parameters are expected to derive from soft masses at higher scales, and the weak-scale parameters are determined by the more fundamental high energy parameters by renormalization group evolution.

In practice, the renormalization group flow will be inverted. Measurements of superpartner properties at the linear collider and the LHC will provide determinations of weak-scale supersymmetry parameters. These can then be evolved to high scales to determine their fundamental, microscopic values.

An example of the power of this approach is given in Fig. 27 [96]. The assumed physical framework is a minimal supergravity model. The precision with which the weak-scale parameters will be measured depends, of course, on the collider and detector parameters. The authors assumed mass precisions of

$$
\begin{aligned}
\Delta m_{\tilde{\chi}^{ \pm, 0}} & \approx 0.3 \mathrm{GeV} \\
\Delta m_{\tilde{l}} \approx \Delta m_{\tilde{\nu}} & \approx 0.1 \mathrm{GeV} \\
\Delta m_{\tilde{\tau}} & \approx 0.6 \mathrm{GeV} \\
\Delta m_{\tilde{t}, \tilde{b}} & \approx 1 \mathrm{GeV} .
\end{aligned}
$$

Along with analyses of cross sections and other quantities in slepton and chargino/neutralino events, these measurements would provide determinations of the gaugino parameters $M_{1}$ and $M_{2}$ at the percent level. The gluino mass $M_{3}$ is assumed to be measured by a combination of LHC and linear collider data. Note that the linear collider information improves the $M_{3}$ 

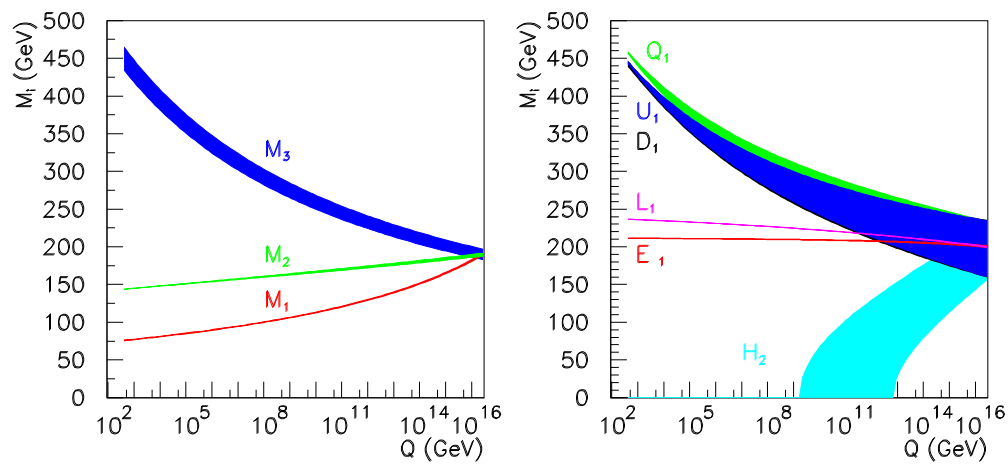

Fig. 27. Renormalization group evolution of gaugino (left) and sfermion (right) mass parameters from the weak scale to the GUT scale in a minimal supergravity model with $m_{0}=200 \mathrm{GeV}, M_{1 / 2}=190 \mathrm{GeV}, A_{0}=500 \mathrm{GeV}, \tan \beta=30$, and $\mu<0$ 96. $H_{u}$ denotes the $m_{H_{u}}$ renormalization group trajectory. The weak-scale experimental inputs are discussed in the text, and the bands indicate 95\% CL contours.

precision by over an order of magnitude through the complementarity described in Sec. (5.2).

Extrapolations of the supersymmetry parameters from the weak scale to the GUT scale are shown in Fig. 27. The unification of gaugino masses is striking, providing clear evidence for gauge group unification. The sfermion masses also unify at the same scale, with great precision for the sleptons. The quark and Higgs parameters are less well-determined, and the uncertainty in weak-scale values is noticeably magnified as one evolves to higher energies. Nevertheless, taken as a whole, the sfermion mass trajectories also show clear evidence for unification at the GUT scale.

The identical analysis has been performed for a gauge-mediated supersymmetry breaking model. The results are show in Fig. 28. Recall that the simplest gauge-mediated models predict gaugino mass relations identical to those in unified scenarios, but the scalar mass predictions of Eq. (57) are very different. The results of Fig. 28 clearly disfavor scalar mass unification at any scale, and would thereby exclude minimal supergravity and many GUTs. In addition, the renormalization group trajectories show unification of the the left-handed slepton $L_{1}$ and $H_{u}$ masses at $10^{8} \mathrm{GeV}$. In gauge-mediated scenarios this identifies the messenger scale. 


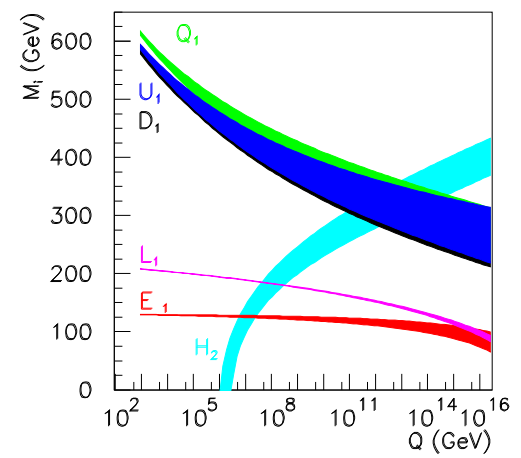

Fig. 28. Evolution of sfermion mass parameters in a gauge-mediated supersymmetry breaking model with $M_{\text {mess }}=2 \times 10^{8} \mathrm{GeV}, \Lambda=\langle F\rangle / M_{\text {mess }}=28 \mathrm{TeV}, N=3, \tan \beta=30$ and $\mu<0$ 96. $H_{u}$ denotes the $m_{H_{u}}$ renormalization group trajectory.

\section{Connections to Cosmology}

The discovery of supersymmetry with a potentially stable LSP implies the discovery of dark matter candidates. These discoveries will open many avenues for dark matter studies, with a wealth of connections between particle physics and cosmology. A schematic picture of the resulting investigation of supersymmetric dark matter is given in Fig. 29.

In the MSSM, suitable dark matter candidates are the neutralino and the gravitino. In the case of neutralino dark matter, the thermal relic density is determined by the neutralino pair annihilation cross section as described in Sec. (3.2), and dark matter may be detected either directly through its interactions with ordinary matter or indirectly through its annihilation decay products. At linear colliders, supersymmetry parameters may be determined at the percent level, and the thermal relic density and neutralinonucleon scattering cross sections may be determined with similar precision. Such progress corresponds to the upper-half of Fig. 29, and the completion of this program will provide a great deal of information about the suitability of neutralinos as dark matter candidates.

As examples, recall that the thermal relic density and detection rates are sensitive to gaugino-Higgsino mixing, as explained in Secs. (3.2) and (4.2). The mixing depends on the ratios $M_{i} /|\mu|$ and may be measured either by studying both chargino states, if available, or by studying a single chargino state with polarized beams, as discussed in Sec. (6.2). The relic density is also modified if there are other superpartners with masses within roughly $5 \%$ of the LSP mass [97]. In the case of minimal supergravity, for example, 


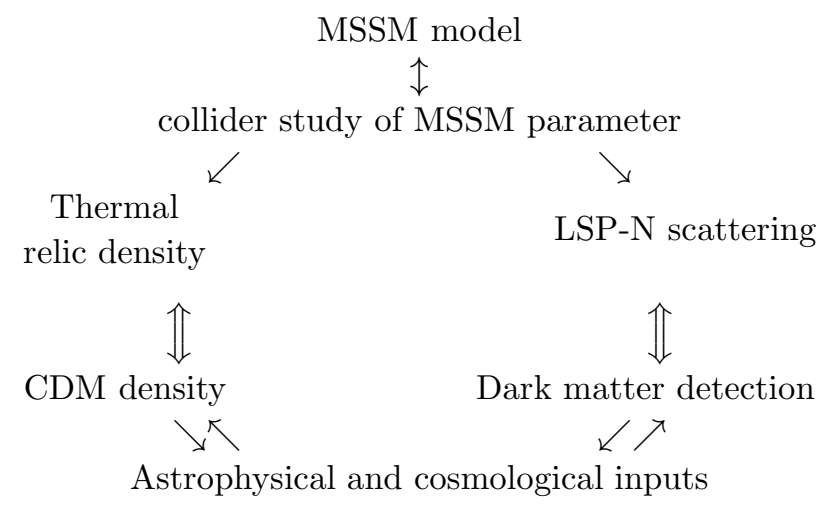

Fig. 29. The road to understanding supersymmetric dark matter.

the lighter $\tilde{\tau}$ is lighter than all other charged superpartners and may be nearly degenerate with the neutralino LSP. In this case, the thermal relic density is reduced by coanihilation effects and is highly sensitive to the $\tilde{\tau}-\tilde{\chi}_{1}^{0}$ mass splitting. Precise mass measurements are therefore needed to pin down the relic density. The mass determination of highly degenerate sparticles is very challenging in general; in this case, it is complicated by

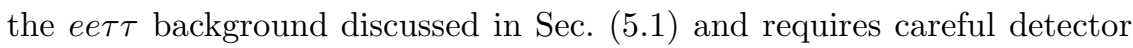
design to retain sensitivity to low momentum jets and leptons.

Even if the supersymmetry parameters are all precisely measured, this is not the whole story, however. The identification of the thermal relic density with the present day cold dark matter density is subject to cosmological assumptions. For example, the calculation of the thermal relic density assumes that the dominant source of dark matter is from dark matter particles falling out of thermal equilibrium. It is possible, however, that the bulk of the dark matter is created not through thermal equilibrium and freeze-out, but through the decay of a supermassive particle after freeze-out, but before $t=1 \mathrm{~s}$. For example, in the anomaly-mediated supersymmetry breaking models of Sec. (4.5), the LSP thermal relic density is negligible, as Winos annihilate extremely efficiently in the early universe. However, anomaly-mediated scenarios also have supermassive gravitinos, with mass $\sim 10 \mathrm{TeV}$. These may decay to LSPs after the usual freeze-out temperatures, producing Wino LSPs with a cosmologically interesting mass density [98].

The thermal relic density calculation also assumes that nothing unusual happens once the dark matter is produced at temperatures of $T \sim$ 
$\mathcal{O}(10) \mathrm{GeV}$. At present, the thermal history of the universe is on sure footing only for times after Big Bang nucleosynthesis, that is, times $t \gtrsim 1 \mathrm{~s}$ and temperatures $T \lesssim 1 \mathrm{MeV}$. The thermal relic density calculation therefore requires an extrapolation of four orders of magnitude in temperature. Large entropy production by late-decaying particles may drastically alter calculated relic densities, reducing a seemingly too-large relic density to the ideal range, for example. The bottom line is that the cold dark matter density obtained following the path from the bottom of Fig. 29 need not coincide with the thermal relic density obtained by following the path from the top. Instead, discrepancies might provide new insights into the history of our universe.

In a similar vein, the neutralino-nucleon cross sections are not necessarily in one-to-one correspondence with dark matter detection rates. This correspondence requires information about the local density and velocities of dark matter. The uncertainties and problems associated with these issues have been discussed extensively [99, 100, 101. If neutralinos are identified as the dark matter, future colliders will determine their properties and the neutralino-nucleon scattering cross sections. To the extent that these may be checked against actual detection rates, future colliders will also provide important information about dark matter halo densities and velocity distributions.

The second possible supersymmetric dark matter candidate is the gravitino. Gravitino dark matter is somewhat beyond the scope of this paper; for an excellent review, see Ref. 42]. Recall, however, that the NLSP lifetime is related to the gravitino mass, as both are determined by $F_{\mathrm{DSB}}$ as in Eqs. (39) and (40). The gravitino's relic density is proportional to the gravitino's mass, and so the observed dark matter density provides an upper limit on the gravitino's mass of $m_{3 / 2} \lesssim 0.2 \mathrm{keV}$ typically. In this range, the gravitino is hot dark matter. Gravitinos with masses around $1 \mathrm{keV}$ may be interesting warm dark matter candidates. In this range, the displaced vertices of NLSP decays to gravitinos may be accessible at linear colliders through the studies discussed in Sec. (8).

\section{Conclusions}

Supersymmetry and the linear collider are a near perfect fit - it is difficult to envision a richer linear collider program than that provided by the superpartner spectrum, and it is hard to imagine a more incisive tool for studying supersymmetry than the linear collider. Of course, the program of 
supersymmetry studies at the linear collider depends on what superpartners are kinematically accessible. However, as evident from the many examples discussed above, even if only one or a few superpartners are kinematically accessible, the linear collider will be able to provide model-independent measurements of a host of soft supersymmetry-breaking parameters. In conjunction with the information provided by hadron colliders, this information will likely provide precise information about sparticles beyond the linear collider's reach.

In this overview, we have highlighted many of the fundamental questions that will be addressed by such supersymmetry measurements. These range from issues specific to supersymmetry, such as the testing of supersymmetric identities, the resolution of the supersymmetric flavor and $\mathrm{CP}$ puzzles, and the determination of the scale of supersymmetry breaking, to grand universal questions, such as the nature of dark matter, the unification of forces, and the geometry of spacetime. As discussed in this review, if supersymmetry is within reach, the linear collider may shed light on all of these issues, and will, in some cases, provide definitive answers.

At the same time, there are many outstanding problems. To name but a few, the study of radiative corrections in supersymmetry and its impact on precision measurements is still in its early days. In addition, the importance of experimental uncertainties, such as in the luminosity spectrum, beam energy, and polarimetry have been considered in a few studies, but have not been systematically investigated. And of course, new ideas for physics beyond the standard model continually arise, and the potential of linear colliders to probe such new ideas and to differentiate these ideas from other new physics possibilities will continue to be of interest. To fulfill the potential of linear colliders to study new physics, dedicated experimental and theoretical efforts are still needed. We hope this chapter will be of use to people who are interested in continuing the exploration of supersymmetry at new colliders.

\section{Acknowledgments}

We thank our collaborators for their many insights through the years, Keisuke Fujii, David Miller, and Amarjit Soni for their support of this review, and Michael Peskin for sparking our initial interest in this subject. 


\section{References}

1. J. Bagger and J. Wess, Supersymmetry And Supergravity, Princeton University Press, Princeton, NJ (1990).

2. H. E. Haber, "Introductory low-energy supersymmetry," hep-ph/9306207.

3. J. A. Bagger, "Weak-scale supersymmetry: Theory and practice," hep$\mathrm{ph} / 9604232$

4. M. Drees, "An introduction to supersymmetry," hep-ph/9611409.

5. X. Tata, "What is supersymmetry and how do we find it?," hep-ph/9706307.

6. S. P. Martin, "A supersymmetry primer," in Perspectives on Supersymmetry, ed. G. L. Kane, pp. 1-98 hep-ph/9709356.

7. S. Weinberg, The Quantum Theory Of Fields. Vol. 3: Supersymmetry, Cambridge University Press, Cambridge, UK (2000).

8. N. Polonsky, Supersymmetry: Structure and phenomena. Extensions of the standard model, Lect. Notes Phys. M68, 1 (2001) hep-ph/0108236.

9. T. Abe et al. [American Linear Collider Working Group Collaboration], hep-ex/0106055; hep-ex/0106056; hep-ex/0106057; hep-ex/0106058

10. J. A. Aguilar-Saavedra et al. [ECFA/DESY LC Physics Working Group Collaboration], hep-ph/0106315.

11. K. Abe et al. [ACFA Linear Collider Working Group Collaboration], hep$\mathrm{ph} / 0109166$.

12. J. L. Feng and D. E. Finnell, Phys. Rev. D49, 2369 (1994) hep-ph/9310211.

13. H. Baer, R. Munroe and X. Tata, Phys. Rev. D 54, 6735 (1996) [Erratumibid. D 56, 4424 (1997)] [hep-ph/9606325.

14. A. Bartl, H. Eberl, S. Kraml, W. Majerotto, W. Porod and A. Sopczak, Z. Phys. C 76, 549 (1997) hep-ph/9701336.

15. M. Drees and O. J. Eboli, Eur. Phys. J. C 10, 337 (1999) hep-ph/9902391.

16. A. Bartl, H. Eberl, S. Kraml, W. Majerotto and W. Porod, Eur. Phys. J. directC 6, 1 (2000) hep-ph/0002115.

17. R. Kitano, T. Moroi and S. Su, hep-ph/0208149.

18. K. R. Dienes, Phys. Rept. 287, 447 (1997) hep-th/9602045.

19. P. Horava and E. Witten, Nucl. Phys. B 475, 94 (1996) hep-th/9603142.

20. H. Goldberg, Phys. Rev. Lett. 50, 1419 (1983).

21. L. M. Krauss, Nucl. Phys. B 227, 556 (1983).

22. J. Ellis, J. S. Hagelin, D. V. Nanopoulos and M. Srednicki, Phys. Lett. B127, 233 (1983).

23. G. Jungman, M. Kamionkowski and K. Griest, Phys. Rept. 267, 195 (1996) hep-ph/9506380.

24. F. Gabbiani, E. Gabrielli, A. Masiero and L. Silvestrini, Nucl. Phys. B 477, 321 (1996) hep-ph/9604387.

25. R. Contino and I. Scimemi, Eur. Phys. J. C 10, 347 (1999) hep-ph/9809437.

26. T. Moroi, Phys. Rev. D 53, 6565 (1996) [Erratum-ibid. D 56, 4424 (1997)] hep-ph/9512396.

27. T. Goto and T. Nihei, Phys. Rev. D 59, 115009 (1999) hep-ph/9808255.

28. K. S. Babu and M. J. Strassler, hep-ph/9808447

29. T. Goto and T. Nihei, hep-ph/9909251. 
30. Y. Suzuki et al. [TITAND Working Group Collaboration], hep-ex/0110005.

31. A. H. Chamseddine, R. Arnowitt and P. Nath, Phys. Rev. Lett. 49, 970 (1982)

32. R. Barbieri, S. Ferrara and C. A. Savoy, Phys. Lett. B 119, 343 (1982).

33. L. J. Hall, J. Lykken and S. Weinberg, Phys. Rev. D 27, 2359 (1983).

34. J. L. Feng, K. T. Matchev and F. Wilczek, Phys. Lett. B 482, 388 (2000) hep-ph/0004043.

35. J. L. Feng, K. T. Matchev and T. Moroi, Phys. Rev. Lett. 84, 2322 (2000) hep-ph/9908309.

36. J. L. Feng, K. T. Matchev and T. Moroi, Phys. Rev. D 61, 075005 (2000) hep-ph/9909334.

37. J. L. Feng and K. T. Matchev, Phys. Rev. D 63, 095003 (2001) hep$\mathrm{ph} / 0011356$.

38. M. Drees, Phys. Rev. D33, 1468 (1986); M. Dine, A. Kagan and S. Samuel, Phys. Lett. B243, 250 (1990); S. Dimopoulos and G. F. Giudice, Phys. Lett. B357, 573 (1995) hep-ph/9507282; A. Pomarol and D. Tommasini, Nucl. Phys. B466, 3 (1996) hep-ph/9507462].

39. G. Dvali and A. Pomarol, Phys. Rev. Lett. 77, 3728 (1996) hepph/9607383; A. G. Cohen, D. B. Kaplan and A. E. Nelson, Phys. Lett. B388, 588 (1996) hep-ph/9607394.

40. J. L. Feng, C. Kolda and N. Polonsky, Nucl. Phys. B546, 3 (1999) hepph/9810500]; J. Bagger, J. L. Feng and N. Polonsky, Nucl. Phys. B563, 3 (1999) hep-ph/9905292; J. A. Bagger, J. L. Feng, N. Polonsky and R. Zhang, Phys. Lett. B473, 264 (2000) hep-ph/9911255; H. Baer, C. Balazs, P. Mercadante, X. Tata and Y. Wang, Phys. Rev. D63, 015011 (2001) hep-ph/0008061; H. Baer, C. Balazs, M. Brhlik, P. Mercadante, X. Tata and Y. Wang, hep-ph/0102156; J. Hisano, K. Kurosawa and Y. Nomura, Phys. Lett. B445, 316 (1999) [hep-ph/9810411]; Nucl. Phys. B584, 3 (2000) hep-ph/0002286.

41. M. Dine and A. E. Nelson, Phys. Rev. D 48, 1277 (1993) hep-ph/9303230; M. Dine, A. E. Nelson and Y. Shirman, Phys. Rev. D 51, 1362 (1995) hepph/9408384; M. Dine, A. E. Nelson, Y. Nir and Y. Shirman, Phys. Rev. D 53, 2658 (1996) hep-ph/9507378.

42. G. F. Giudice and R. Rattazzi, Phys. Rept. 322, 419 (1999) hep$\mathrm{ph} / 9801271$.

43. S. Dimopoulos, G. R. Dvali, R. Rattazzi and G. F. Giudice, Nucl. Phys. B 510, 12 (1998) hep-ph/9705307.

44. J. L. Feng and T. Moroi, Phys. Rev. D 58, 035001 (1998) hep-ph/9712499.

45. D. R. Stump, M. Wiest and C. P. Yuan, Phys. Rev. D 54, 1936 (1996) hep-ph/9601362; S. Dimopoulos, M. Dine, S. Raby and S. Thomas, Phys. Rev. Lett. 76, 3494 (1996) hep-ph/9601367.

46. L. Randall and R. Sundrum, Nucl. Phys. B 557, 79 (1999) hep-th/9810155.

47. G. F. Giudice, M. A. Luty, H. Murayama and R. Rattazzi, JHEP 9812, 027 (1998) hep-ph/9810442.

48. T. Gherghetta, G. F. Giudice and J. D. Wells, Nucl. Phys. B 559, 27 (1999) hep-ph/9904378; J. L. Feng and T. Moroi, Phys. Rev. D 61, 095004 (2000) 
hep-ph/9907319.

49. See, e.g., A. Pomarol and R. Rattazzi, JHEP 9905, 013 (1999) hepph/9903448; Z. Chacko, M. A. Luty, I. Maksymyk and E. Ponton, JHEP 0004, 001 (2000) hep-ph/9905390; E. Katz, Y. Shadmi and Y. Shirman, JHEP 9908, 015 (1999) hep-ph/9906296; I. Jack and D. R. Jones, Phys. Lett. B 482, 167 (2000) hep-ph/0003081].

50. C. H. Chen, M. Drees and J. F. Gunion, Phys. Rev. Lett. 76, 2002 (1996) hep-ph/9512230; Phys. Rev. D 55, 330 (1997) [Erratum-ibid. D 60, 039901 (1999)] [hep-ph/9607421]; S. Thomas and J. D. Wells, Phys. Rev. Lett. 81, 34 (1998) hep-ph/9804359]; H. C. Cheng, B. A. Dobrescu and K. T. Matchev, Nucl. Phys. B 543, 47 (1999) hep-ph/9811316; J. L. Feng, T. Moroi, L. Randall, M. Strassler and S. f. Su, Phys. Rev. Lett. 83, 1731 (1999) hep-ph/9904250; J. F. Gunion and S. Mrenna, Phys. Rev. D 64, 075002 (2001) hep-ph/0103167.

51. V. S. Kaplunovsky and J. Louis, Phys. Lett. B 306, 269 (1993) hepth/9303040; A. Brignole, L. E. Ibanez, C. Munoz and C. Scheich, Z. Phys. C 74, 157 (1997) hep-ph/9508258; T. Kobayashi, D. Suematsu, K. Yamada and Y. Yamagishi, Phys. Lett. B 348, 402 (1995) hep-ph/9408322; A. Brignole, L. E. Ibanez and C. Munoz, Nucl. Phys. B 422, 125 (1994) [Erratum-ibid. B 436, 747 (1995)] hep-ph/9308271].

52. R. Barbieri and L. J. Hall, Phys. Lett. B 338, 212 (1994) hep-ph/9408406; R. Barbieri, L. J. Hall and A. Strumia, Nucl. Phys. B 445, 219 (1995) hep-ph/9501334.

53. M. Drees, Phys. Lett. B 181, 279 (1986).

54. Y. Kawamura, H. Murayama and M. Yamaguchi, Phys. Rev. D 51, 1337 (1995) hep-ph/9406245.

55. See, e.g., K. Huitu, J. Maalampi and K. Puolamaki, Eur. Phys. J. C 6, 159 (1999) hep-ph/9705406; S. Bar-Shalom, G. Eilam and A. Soni, Phys. Rev. Lett. 80, 4629 (1998) hep-ph/9802251]; D. K. Ghosh, R. M. Godbole and S. Raychaudhuri, hep-ph/9904233; M. Besancon and G. Moreau, hepph/9909441: D. K. Ghosh and S. Roy, Phys. Rev. D 63, 055005 (2001) hep-ph/0003225.

56. JLC Group Collaboration, "JLC-I," KEK-92-16.

57. T. Tsukamoto, K. Fujii, H. Murayama, M. Yamaguchi and Y. Okada, Phys. Rev. D 51, 3153 (1995).

58. H. U. Martyn and G. A. Blair, hep-ph/9910416.

59. M. Dima et al., Phys. Rev. D 65, 071701 (2002) hep-ex/0112017.

60. David Wagner, http://hep-www.colorado.edu/SUSY, COLO-HEP-400, COLO-HEP-401.

61. J. D. Lykken, hep-ph/9803427.

62. J. L. Feng, Int. J. Mod. Phys. A 13, 2319 (1998) hep-ph/9803319.

63. H. U. Martyn, hep-ph/0002290.

64. J. L. Feng and M. E. Peskin, Phys. Rev. D 64, 115002 (2001) hep$\mathrm{ph} / 0105100$.

65. A. Freitas, D. J. Miller and P. M. Zerwas, Eur. Phys. J. C 21, 361 (2001) hep-ph/0106198. 
66. J. K. Mizukoshi, H. Baer, A. S. Belyaev and X. Tata, Phys. Rev. D 64, 115017 (2001) hep-ph/0107216.

67. C. Blochinger, H. Fraas, G. Moortgat-Pick and W. Porod, Eur. Phys. J. C 24, 297 (2002) hep-ph/0201282].

68. V. Barger, T. Han and J. Kelly, Phys. Lett. B419, 233 (1998) hep$\mathrm{ph} / 9709366$.

69. I. Hinchliffe, F. E. Paige, M. D. Shapiro, J. Soderqvist and W. Yao, Phys. Rev. D 55, 5520 (1997) hep-ph/9610544.

70. H. Bachacou, I. Hinchliffe and F. E. Paige, Phys. Rev. D 62, 015009 (2000) hep-ph/9907518.

71. ATLAS Collaboration, ATLAS Detector and Physics Performance Technical Design Report, CERN/LHCC/99-14, http://atlasinfo.cern.ch/Atlas/GROUPS/PHYSICS/TDR/access.html.

72. B. C. Allanach, C. G. Lester, M. A. Parker and B. R. Webber, JHEP 0009, 004 (2000) hep-ph/0007009.

73. M. M. Nojiri, K. Fujii and T. Tsukamoto, Phys. Rev. D 54, 6756 (1996) hep-ph/9606370.

74. M. M. Nojiri, Phys. Rev. D 51, 6281 (1995) hep-ph/9412374.

75. N. Arkani-Hamed, H. C. Cheng, J. L. Feng and L. J. Hall, Phys. Rev. Lett. 77, 1937 (1996) hep-ph/9603431.

76. N. Arkani-Hamed, J. L. Feng, L. J. Hall and H. C. Cheng, Nucl. Phys. B 505, 3 (1997) hep-ph/9704205; D. Bowser-Chao and W. Y. Keung, Phys. Rev. D 56, 3924 (1997) thep-ph/9704219]; M. Hirouchi and M. Tanaka, Phys. Rev. D 58, 032004 (1998) hep-ph/9712532]; J. Hisano, M. M. Nojiri, Y. Shimizu and M. Tanaka, Phys. Rev. D 60, 055008 (1999) hepph/9808410]; J. J. Cao, T. Han, X. Zhang and G. R. Lu, Phys. Rev. D 59, 095001 (1999) hep-ph/9808466]; D. Nomura, Phys. Rev. D 64, 075001 (2001) hep-ph/0004256]; M. Guchait, J. Kalinowski and P. Roy, Eur. Phys. J. C 21, 163 (2001) hep-ph/0103161; M. Dine, Y. Grossman and S. Thomas, hep-ph/0111154: W. Porod and W. Majerotto, Phys. Rev. D 66, 015003 (2002) hep-ph/0201284].

77. J. L. Feng, M. E. Peskin, H. Murayama and X. Tata, Phys. Rev. D 52, 1418 (1995) hep-ph/9502260.

78. S. Y. Choi, A. Djouadi, H. K. Dreiner, J. Kalinowski and P. M. Zerwas, Eur. Phys. J. C 7, 123 (1999) hep-ph/9806279; S. Y. Choi, A. Djouadi, H. S. Song and P. M. Zerwas, Eur. Phys. J. C 8, 669 (1999) hepph/9812236 ; S. Y. Choi, M. Guchait, J. Kalinowski and P. M. Zerwas, Phys. Lett. B 479, 235 (2000) hep-ph/0001175]; S. Y. Choi, A. Djouadi, M. Guchait, J. Kalinowski, H. S. Song and P. M. Zerwas, Eur. Phys. J. C 14, 535 (2000) hep-ph/0002033; see also D. Atwood, S. Bar-Shalom, G. Eilam and A. Soni, Phys. Rept. 347, 1 (2001) hep-ph/0006032.

79. G. Moortgat-Pick, A. Bartl, H. Fraas and W. Majerotto, hep-ph/0004181; Eur. Phys. J. C 18, 379 (2000) hep-ph/0007222].

80. T. Ibrahim and P. Nath, Phys. Rev. D 58, 111301 (1998) [Erratum-ibid. D 60, 099902 (1999)] [hep-ph/9807501.

81. S. Y. Choi, J. Kalinowski, G. Moortgat-Pick and P. M. Zerwas, Eur. Phys. 
J. C 22, 563 (2001) hep-ph/0108117; Addendum, Eur. Phys. J. C 23, 769 (2001) hep-ph/0202039.

82. V. D. Barger, T. Falk, T. Han, J. Jiang, T. Li and T. Plehn, Phys. Rev. D 64, 056007 (2001) hep-ph/0101106.

83. H. C. Cheng, K. T. Matchev and M. Schmaltz, Phys. Rev. D 66, 056006 (2002) hep-ph/0205314.

84. H. C. Cheng, J. L. Feng and N. Polonsky, Phys. Rev. D 56, 6875 (1997) hep-ph/9706438.

85. H. C. Cheng, J. L. Feng and N. Polonsky, Phys. Rev. D 57, 152 (1998) hep-ph/9706476.

86. M. M. Nojiri, D. M. Pierce and Y. Yamada, Phys. Rev. D 57, 1539 (1998) hep-ph/9707244.

87. E. Katz, L. Randall and S. Su, Nucl. Phys. B536, 3 (1998) hep$\mathrm{ph} / 9801416$.

88. S. Kiyoura, M. M. Nojiri, D. M. Pierce and Y. Yamada, Phys. Rev. D 58, 075002 (1998) hep-ph/9803210.

89. U. Mahanta, Phys. Rev. D59, 015017 (1999) hep-ph/9810344.

90. P. H. Chankowski, Phys. Rev. D41, 2877 (1990).

91. K. Hikasa and Y. Nakamura, Z. Phys. C70, 139 (1996) hep-ph/9501382; Erratum, Z. Phys. C71, 356 (1996).

92. M. E. Peskin and T. Takeuchi, Phys. Rev. Lett. 65, 964 (1990).

93. J. Bagger, K. T. Matchev and D. Pierce, Phys. Lett. B 348, 443 (1995) hepph/9501277|; D. M. Pierce, J. A. Bagger, K. T. Matchev and R. j. Zhang, Nucl. Phys. B 491, 3 (1997) hep-ph/9606211; T. Fritzsche and W. Hollik, Eur. Phys. J. C 24, 619 (2002) hep-ph/0203159.

94. M. A. Diaz, S. F. King and D. A. Ross, Nucl. Phys. B 529, 23 (1998) hep-ph/9711307; M. A. Diaz, S. F. King and D. A. Ross, Phys. Rev. D 64, 017701 (2001) hep-ph/0008117; M. A. Diaz and D. A. Ross, hepph/0205257; J. Guasch, W. Hollik and J. Sola, hep-ph/0207364.

95. S. Ambrosanio and G. A. Blair, Eur. Phys. J. C 12, 287 (2000) hepph/9905403.

96. G. A. Blair, W. Porod and P. M. Zerwas, Phys. Rev. D 63, 017703 (2001) hep-ph/0007107; G. A. Blair, W. Porod and P. M. Zerwas, hep$\mathrm{ph} / 0210058$.

97. K. Griest and D. Seckel, Phys. Rev. D 43, 3191 (1991).

98. T. Moroi and L. Randall, Nucl. Phys. B 570, 455 (2000) hep-ph/9906527.

99. P. Sikivie, I. I. Tkachev and Y. Wang, Phys. Rev. D 56, 1863 (1997) astro$\mathrm{ph} / 9609022$.

100. G. Gelmini and P. Gondolo, Phys. Rev. D 64, 023504 (2001) hep$\mathrm{ph} / 0012315$.

101. C. Calcaneo-Roldan and B. Moore, Phys. Rev. D 62, 123005 (2000) astroph/0010056. 


\section{INDEX}

$A$-terms, 10

$B$-terms, 10

$D$-terms, 9

$F$-terms, 7

$R$-parity and dark matter, 17 and proton decay, 9 conservation, 9, 17 definition, 9 violating terms, 8 violation, 10,34

$e^{-} e^{-}$option, uses for

flavor measurements, 46 mass measurements, 39 tests of supersymmetry, 58 $\mu$ parameter, definition, 7 $\tan \beta$

definition, 13

measurement

from $\tilde{l}_{L}-\tilde{\nu}_{l}$ splitting, 39

from staus, 45, 49

auxiliary fields, 5

beam polarization difficulty for Winos, 51

power of, 42,52

use for charginos, 51

use for sleptons, 35

use in flavor measurements, 46

charginos, 13, 14

backgrounds, 50

decay, 50

mass matrix, 14 mass measurements, 52

mixing matrices, 14

mixing measurements, 52

production, 50

signals, 50

collider parameters, 3, 68

complementarity

of LC and LHC, 40, 64

of low and high energy experiments, 46

of particle and astroparticle physics, 17, 65

of polarized and unpolarized measurements, 52

constraints

$\Delta m_{K}, 17$

$\epsilon_{K}, 19$

electron EDM, 19, 54

lepton flavor violation, 19

neutron EDM, 19, 54

proton decay, 19

cosmology, 65

$\mathrm{CP}$ violation, 54

and baryogenesis, 54

measurement

through $\mathrm{CP}$-conserving quantities, 54

through CP-violating quantities, 56

dark matter, 17, 65

and LSP, 17

annihilation, 18

Bino-like, 25

co-annihilation, 65 
colliders and, 17

detection, 18, 67

gaugino-Higgsino, 27

gravitino, 67

in anomaly-mediation, 66

in focus point supersymmetry, 27

non-thermal production, 66

thermal relic density, 17, 65

velocity distribution, 67

Wino-like, 66

displaced vertices, 62

electroweak symmetry breaking, 13

radiative, 24

tree relations, 24

flavor violation

is generic, 12

lepton, 45

collider signals, 46

low energy, 19, 46

frustration

of invisible neutralinos, 50

gaugino masses, 10

gauginos, 6

gluinos, 11

Grassmanian coordinates, 4

gravitino, 6

as dark matter, 67

interactions, 22

mass, 22

in anomaly-mediation, 32

in gauge-mediation, 30

in supergravity, 24

measurement, 62

Higgsinos, 6

highly-ionizing charged tracks, 30

interactions

bilinear scalar, 10

dimension 3 but not soft, 10

gauge (gaugino)-matter, 9

matter, 7

pure gauge, 9 trilinear scalar, 10

LSP (lightest supersymmetric particle), 9, 17

as dark matter, 17

in minimal supergravity, 26

messenger

fields, 29

scale, 29,31

measurement, 64

models, 21

anomaly-mediation, 30

and tachyonic sleptons, 32

motivation, 23

typical spectrum, 32

dilaton-dominated, 33

focus point supersymmetry, 25

and super-oblique parameters, 61

typical spectrum, 28

gauge-mediation, 28

fundamental parameters, 30

motivation, 23

NLSP in, 31

signals, 30,62

typical spectrum, 30

GUT, 32

minimal supergravity, 23

fundamental parameters, 24

typical spectrum, 25

string, 32

supergravity, 22

superheavy supersymmetry, 28

and super-oblique parameters, 61

typical spectrum, 28

MSSM (minimal supersymmetric

standard model)

anomaly cancelation, 5

definition, 4

particle content, 6

soft terms, 10

superpotential, 8

neutralinos, 13, 14 
backgrounds, 50

decay, 50

mass matrix, 14

mixing matrix, 14

production, 50

signals, 50

Planck scale

discrepancy with GUT scale, 15

extrapolation to, 63

in gauge-mediation, 64

in minimal supergravity, 63

Poincare algebra, 4

problems

gauge hierarchy, 8-10, 25

outstanding, 68

standard model flavor, 45

supersymmetric CP, 19, 54

supersymmetric flavor, 17

radiative corrections, 61,68

renormalization group

equations, 23

evolution, 63

trajectories, 15

in focus point supersymmetry, 27

scalar masses, 10

sleptons, 5, 6, 11

$\tilde{l}_{L}-\tilde{\nu}_{l}$ mass splitting, 13

measurement, 53

background, 34

decay, 34

flavor mixing

at $e^{-} e^{-}$collider, 46

at $e^{+} e^{-}$collider, 46

mass matrix

$2 \times 2$ form, 12

$3 \times 3$ form, 11

$6 \times 6$ form, 11

mass measurement

at LHC, 39

by $e^{-} e^{-}$threshold scan, 39

by $e^{+} e^{-}$threshold scan, 38

by kinematic endpoints, 36 by minimum mass method, 38 mixing

flavor, 12, 45

left-right, 12

production, 34

polarized, 41

signal, 34

spin measurement, 39

sneutrinos

mass matrix, 12

mass measurement, 53

squarks, 5, 6, 11

staus

left-right mixing, 44

lightness of, 44

mass eigenstates, 13

mass matrix, 12

production, 44

signals, 47

super-oblique

corrections, 59

analogy to oblique, 59

parameters, 59

and focus point supersymmetry,

61

and superheavy supersymmetry, 61

non-decoupling of, 60

supercurrent, 6

superfields, 5

chiral, 5

vector, 6

superpartners

coupling equivalence of, 9

mass degeneracy of, 7, 10

superpotential, 7

supersymmetry

algebra, 4

as an extra dimension, 5

operators, 5

supersymmetry breaking

dynamical, 21

hard, 59

mediation mechanism, 22

necessity of, 7,10

scale of, 21,22 
determination of, 61

soft terms, 10

origin of, 21

tests

of $\tilde{l}_{L}-\tilde{\nu}_{l}$ mass relations, 13, 39, 53

of chirality preservation in gaugino interactions, 42

of gaugino Majorana-ness, 43

of gaugino mass unification, 53, 64

of scalar mass unification, 38, 44,

64

of slepton spin, 39

of supersymmetry itself, 56

time-of-flight signatures, 30

two-photon background, 36

unification

and proton decay, 20

gauge coupling, 15

signficance of, 15

gaugino mass, 16

matter multiplet, 33

matter representations, 16

upper bounds

from super-oblique parameters, 61

from Wino-Higgsino mixing, 54

on sneutrino mass, 54 